
SOME NEW REACTIONS AND DERIVATIVES OF KOJIC ACID -BEÉLIK 
SOME NEW REACTIONS AND DERIVATIVES

OF KOJIC ACID

\begin{abstract}
A Thesis
Submitted to the Faculty of Graduate Studies and Research in partial fulfilment of the requirements for the degree of Doctor of Philosophy
\end{abstract}

by

Andrew Beélik, M. S. A. (Toronto),

McGill University

August 1954 
ACKNOWLEDGEMENTS

The author wishes to express his sincere gratitude to Professor C. B. Purves for his stimulating direction throughout the course of this research. The valuable assistance of the National Research Council of Canada, in the form of a Studentship (1953-1954), and of the Pulp and Paper Research Institute of Conada, in the form of a sumer grant (1953), is gratefully acknowledged. A generous gift of kojic acid from the corn Products Refining Company is also greatly appreciated. 
TABLE OF CONTENTS

GENERAL INTRODUCTION

vi

HISTORICAL INTRODUCTION

Discovery and Proof of Structure 2

Formation

Biosynthesis

Chemical Synthesis

Properties 28

Chemical Properties 28

Physical Properties 48

Biological Properties 49

DISCUSSION OF RESULTS

Attempted Ring Cleavage of Kojic Acid at Position 1

Analogous Reactions of Other $r$-Pyrones 52

Resistance of Kojic Acid to Aqueous Alkali 55

Methylation Studies 58

Benzoylation Studies 61

Debenzoylation of 5-O-Benzoyl Derivatives of Kojic Acid

by Hydroxylamine 69

Preferential Hydrolysis of Dibenzoylkojic Acid 69

Selective Debenzoylation of Other 5-Benzoates of Kojic Acid 73

Reduction of Dibenzoylkojic Acid 75

Hydrogenation over Platinum 75

Reductive Fission by Zinc in Glacial Acetic Acid 77

Condensations of Kojic Acid and Its Derivatives with

Carbonyl Reagents

79

Analogous Reactions of Other r-Pyxones 79

Negative Results with Some Carbonyl Reagents 81

Open Chain Semicarbazones Derived from Dibenzoylkojic Acid 82 
(a) Action of Cold Aqueous Sodium Hydroxide on Kojic Acid

(b) Preparation of Sodium Kojate

(c) Preparation of 5-Hydroxy-2-(methoxymethyl)- $\gamma$-pyrone and Its Aluminum Salt

(d) Recovery of 5-Hydroxy-2-(methoxymethyl)- $\gamma$-pyrone from Its Aluminum Salt

(e) Methylation of 5-Hydroxy-2-(methoxymethyl)- $\gamma$-pyrone with Diazomethane

(f) Benzoylation of Kojic Acid in Aqueous Sodium Hydroxide I

(g) Benzoylation of Kojic Acid in Aqueous Sodium Hydroxide II

(h) Benzoylation of Kojic Acid in Dry Pyridine I

(i) Benzoylation of Kojic Acid in Dry Pyridine II

(j) Preparation of 5-Benzoxy-2-(triphenylmethoxymethyl)$\gamma$-pyrone

(k) Detritylation of 5-Benzoxy-2-(triphenylmethoxymethyl)$\gamma$-pyrone

(1) Preparation of 5-Benzoxy-2-(hydroxymethyl)- $\gamma$-pyrone from Anhydrous Sodium Kojate

101

(m) Resolution of the Molecular Addition Compound of Dibenzoylkojic Acid and Benzojc Acid

(n) Preferential Debenzoylation of Dibenzoylkojic Acid at Position 5 by Hydroxylamine Hydrochloride in the Presence of Weak Bases

(o) Benzoylation of 2-(Benzoxymethyl )-5-hydroxy- $\gamma$-pyrone in Dry Pyridine

(p) Methylation of 2-(Benzoxymethyl)-5-hydroxy- $\gamma$-pyrone with Diazomethane

(q) Benzoylation of 2-(Hydroxymethyl)-5-methoxy- $\gamma$-pyrone in Dry Pyridine

(r) Preparation of 2-(Acetoxymethyl)-5-benzoxy- $\gamma$-pyrone

(s) Debenzoylation of 2-(Acetoxymethyl)-5-benzoxy- $\gamma$-pyrone

(t) Debenzoylation of 5-Benzoxy-2-(triphenylmethoxymethyl)$\gamma$-pyrone

102

103

105

106

107

107

108

109

(u) Catalytic Hydrogenation of Dibenzoylkojic Acid

109

(v) Preparation of Benzoylallomaltol by the Reductive Fission of Dibenzoylkojic Acid

(w) Debenzoylation of Benzoylallomaltol

111

(x) Benzoylation of Allomaltol in Dry Pyridine

(y) Preparation of Dibenzoylkojic Acid $\alpha$-"Disemicarbazone"

(z) Preparation of Dibenzoylkojic Acid $\beta$ - Disemicarbazone"

(aa) Preparation of Dibenzoylkojic Acid $\alpha$-"Monosemicarbazone"

(bb) Preparation of Dibenzoylkojic Acid $\beta$-"Monosemicarbazone"

(cc) Action of Dilute Acid on Dibenzoylkojic Acid $\alpha-$ "Disemicarbazone"

(dd) Action of Dilute Alkali on Dibenzoylkojic Acid $\alpha-" D i s e m i c a r b a z o n e^{n}$

(ee) Oxidation of Dibenzoylkojic Acid $\alpha$-Disemicarbazone" with Aqueous Potassium Permanganate 
(ff) Oxidation of Dibenzoylkojic Acid $\beta$-"Monosemicarbazone" with Aqueous Potassium Permanganate

(gg) Dibenzoylkojic Acid $\beta$-"Monosemicarbazone" and Semicarbazide Hydrochloride

(hh) Dibenzoylkojic Acid $\beta$-"Monosemicarbazone" and Iydroxylamine Hydrochloride

1 Scheme for the Degradation of the Ethyl Methyl Ether of Kojic Acid, and the Separation and $14 \mathrm{C}$ Assay of the Fragments

2 Infra-Red Spectrum of the Molecular Addition Compound of Dibenzoylkojic Acid and Benzoic Acid, and of an Equimolecular Mixture of Dibenzoylkojic Acid and Benzoic Acid

TABLES

I Carbon Sources for the Biosynthesis of Kojic Acid

II Functional Derivatives of Kojic Acid (a)

III Functional Derivatives of Kojic Acid (b)

IV Nuclear Substitution Products of Kojic Acid

$\nabla$ Mixed Derivatives of Kojic Acid (a)

41

VI Mixed Derivatives of Kojic Acid (b)

VII Treatment of Kojic Acid with Normal Aqueous 
GENERAI INTRODUCTION

Kojic acid can be produced by a microbiological process from carbohydrates, and therefore it might serve as a very useful outlet for industrial and agricultural carbohydrate wastes. Since the acid has not yet found practical applications, the present research is directed toward making such application more probable by elucidating certain aspects of the chemistry of kojic acid where basic knowledge is at present lacking.

These gaps were revealed by a detailed survey of all publications dealing with kojic acid, and this material serves as the Historical Introduction of the thesis. The review has already been accepted in its entirety for publication in Volume 10 of "Advances in Carbohydrate Chemistry". Brief reviews concerning the chemistry of other $\gamma$-pyrones related to the subject matter of the thesis will be found under the appropriate subheadings in the Discussion of Results. For the sake of uniformity, the editorial style of the "Advances" was adopted for the entire thesis.

The thesis first discusses the stability of kojic acid in aqueous alkali, and shows how several new crystalline derivatives can conveniently be made in alkaline solution. A selective de-acylation of kojic 
acid benzoates by meens of hydroxylamine hydrochloride is then described, together with other new derivatives prepared, and also a selective reduction of the primary alcohol group in kojic acid. The thesis closes with the demonstration that the ring in kojic acid can be opened in a condensation with semicarbazide, without scission of the six-membered carbon chain. 


\section{HISTORICAL INTRODUCTION}

Kojic acid, 5-hydroxy-2-(hydroxymethyl)-4H-pyran-4-one* (II), is proauced from carbohydrate sources in an aerobic process by a variety of microorganisms. The acid was discovered in 1907, its structure was established in 1924, and its chemical synthesis from D-glucose was achieved in 1930. Since then, a considerable amount of study has been devoted to the biosynthesis of kojic acid, and numerous publications have dealt with its chemical and biological properties. After nearly half a century, kojic acid remains a scientists' curiosity, without industrial importance. It continues to attract attention, because its formation is the simplest case of the biosynthetic conversion of a sugar to a $\gamma$-pyrone, and a thorough knowledge of this process could reveal much about the biological formation of such complex $\gamma$-pyrone derivatives as flavones, flavonols, and furochromones, which are so abundant in the plant kingdom. Again, kojic acid might serve as the starting material for the synthesis of these substances, many of which are important drugs, e.g. rutin and khellin. The early work on kojic acid is summed up in a review by Barham and Smits (1). Although that 
information is presented here in condensed form only, all the references are included. Subsequent work is treated in somewhat greater detail.

\section{Discovery and Proof of Structure}

Kojic acid was first reported by Saito (2), who isolated it as a crystalline substance from the mycelia of Aspergillus oryzae grown on steamed rice. He assumed the substance to be $\beta$-resorcyl carboxylic acid, but this assumption was later proved wrong. Yabute coined the name of the compound (from "koji" = steamed rice), and by systematic study $(3,4)$ gathered enough evidence to propose two alternative hydroxy- $\gamma$-pyrone structures (I, II) for kojic acid. These structures<smiles>O=c1ccoc(O)c1</smiles>

I<smiles>O=c1ccoc(O)c1</smiles>

IV<smiles></smiles>

II<smiles>O=C1C=COCC1=O</smiles>

$\mathrm{V}, \mathrm{R}=\mathrm{COOH}$ or $\mathrm{CH}_{2} \mathrm{OH}$<smiles>O=c1ccoc(O)c1</smiles>

III<smiles></smiles>

VI

closely resembled those of maltol (III) and comenic acid (IV), respectively, and Yabuta undertook to decide between I and II by converting 
kojic acid into III or IV. He favored structure II, because like IV, but unlike III, kojic acid reacted both with bromine and with a diazonium salt to give nuclear substitution products. In these reactions, kojic acid and comenic acid (IV) presumably reacted through a tautomeric form (V), which could arise from II and IV, but not from I and III. Much of this work had already been published, when Traetta Mosca (5) and Wijkman (6) independently announced the discovery of carbohydrate metabolites, which later proved to be identical with kojic acid. Traetta Mosca and Preti (7), apparently still unaware of Yabuta's investigations, reported later in considerable detail on the properties of their substance, and arrived at the same two alternative structures (I, II) as did Yabuta. They, however, favored the wrong alternative (I), and accordingly proposed the name oxymaltol.

It was Yabuta (8) who finally succeeded in clarifying the structure of kojic acid. Conversion of the hydroxymethyl sidechain to a methyl group by chlorination and reduction of the chloromethyl derivative gave not maltol (III) but an isomer (VI). Yabuta named this isomer allomaltol; structure VI, of course, was only confirmed through subsequent results. Oxidation of the hydroxymethyl to a carboxyl group falled, and the pyridone analog of kojic acid (VII) could not be oxidized to<smiles>O=c1cc[nH]c(O)c1</smiles>

VII<smiles>O=C(O)c1ccnc(O)c1</smiles>

VIII 
comenomic acid (VIII), the pyridone derived from comenic acid (IV). Efforts to convert the carboxyl group of IV or VIII to a hydroxymethyl group were equally unsuccessful. The structural relationship between kojic acid and IV was ultimately established by converting both of these compounds in several steps into 4,5-dihydroxy-2-methylpyridine (IXa), which was merely a tautomeric form of 5-hydroxy-2-methyl-4(1프)pyridone (IXb). This result confirmed structure II, namely 5-hydroxy2-(hydroxymethyl)- $\gamma$-pyrone, for kojic acid, in accord with the original<smiles>Oc1ccnc(O)c1</smiles>

IXa

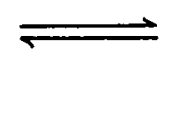

assumption (4). Still more evidence in favor of structure II was furnished by the oxidation of 2-(hydroxymethyl)-5-methoxy- - -pyrone (X) with potassium permanganate to the methyl ether of comenic acid (XI)(9), and by the oxidation of 2-(hydroxymethyl)-5-methoxy-4(1H)-pyridone (XII) to the methyl ether of comenamic acid (XIII) with potassium permanganate (9) or nitric acid (10). The pyridones were prepared by heating the pyrones with concentrated aqueous ammonia under pressure.

It should be noted that this proof of structure depended entirely on the correctness of the structures accepted for comenic acid (IV) and maltol (III). These structures had been assigned by Peratoner and his co-workers $(11,12,13)$ from a set of very reasonable assumptions based 
on conductivity measurements, substitution reactions and alkaline cleavage, but they lacked proof by synthesis. Nevertheless, all these<smiles>O=c1ccocc1O</smiles>

$\mathrm{x}$<smiles>O=c1cc[nH]c2ccc([O-])cc12</smiles>

XII<smiles></smiles>

$\mathrm{XI}$<smiles>O=[R](O)c1cc(=O)c(O)c[nH]1</smiles>

XIII

structures were consistently in good agreement with new reactions and derivatives of the compounds in question, and therefore remained unchallenged. Recently, however, Belonosov (14, 15) brought forward evidence which supposedly favored position 3 for the phenolic hydroxyl group of comenic acid; this in turn suggested structure I for kojic acid. It became desirable to settle this problem in a decisive way. This was accomplished by Heyns and Vogelsang (16), who converted kojic acid via XIII into 5-hydroxypicolinic acid (XVIII) by the following steps: comenamic acid methyl ether (XIII or XIV), 4-chloro-5-methoxypicolinyl chloride (XV), the corresponding acid (XVI), the hydrochloride of 5-methoxypicolinic acid (XVII), and finally XVIII. The position of the 
hydroxyl group in XVIII was established by the synthesis of this compound, using known procedures $(17,18)$, from 2-picoline (XIX) in the following steps: XIX, 2-methyl-5-pyridinesulfonic acid (XX), 2-carboxy5-pyridinesulfonic acid (XXI), and XVIII.<smiles></smiles>

XIII<smiles></smiles>
XVIII $\left\{\begin{array}{l}\mathrm{NaOH} \\ \text { fusion }\end{array}\right.$<smiles>O=C(O)c1ccc(S(=O)(=O)O)cn1</smiles>

$\mathrm{XXI}$<smiles>CCC</smiles><smiles>O=[W]1CCCCC1</smiles><smiles>O=S(=O)(O)c1cccnc1</smiles>

$\mathrm{XX}$

XVII<smiles>CCO[As]1CC[C@H]2CC[C@H]1C2</smiles><smiles>COc1cccc(O)c1</smiles>

XIV<smiles>COc1ccc(C(=O)O)nc1</smiles><smiles>Cc1ccccc1</smiles>

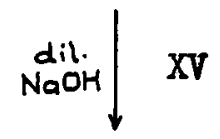<smiles>CC1CC1</smiles><smiles>CC(C)=CC=CC(=O)O</smiles>

$X V I$<smiles>Cc1ccccn1</smiles>

XIX

These results confirmed unequivocally position 5 for the phenolic hydroxyl group in both kojic acid (II) and comenic acid (IV). 


\section{Formation}

\section{Biosynthesis}

The Fermentation:- Kojic acid is produced in an aerobic process by a variety of microorganisms from a wide range of carbon sources. As already mentioned, Saito (2) discovered kojic acid in the mycelium of Aspergillus oryzae grown on steamed rice. Yabuta (3) isolated kojic acid also from the nutrient solutions of A. albus, A. candidus, and A. nidulans; Traetta Mosca (5) claimed to have found it in cultures of A. glaucus; Tamiys and Hida (19) reported its production by A. flavas, A. gymnosardae, A. awamori, A. clavatus, A. fumigatus, and A. giganteus; and Birkinshaw and co-workers (20) showed that it was also formed by A. paragiticus, A. effusus, and A. tamarii. Later, A. luteo-virescens (21), A. lutescens (22), A. wentii (23), and A. alliaceus (23) were added to the list of kojic acid producers. The identity of some of these Aspergilli has been questioned (20). Most members of the A. flavus-oryzae-tamarii group were apparently capable of producing kojic acid, and A. oryzae and A. flavus were possibly most widely used for this purpose. In addition, the formation of kojic acid was also observed in cultures of Penicillium daleae (20), of ten species of acetic acid bacilli (24), and of the gluconic acid fermenter Gluconoacetobacter opacus var. mobilis (25).

Rice and other cereals were the sources of carbon in the early studies $(2,3)$, but they were soon replaced by solutions of pure 
compounds. Microorganisms were grown on media containing a great variety of carbohydrates and related substances, and they produced kojic acid from compounds containing two to seven carbon atoms per molecule, among them reducing sugars, sugar acids and sugar alcohols. Di- and polysaccharides were also suitable substrates. These positive results are collected in Table I; negative results and information about the species of mold used were tabulated in the earlier review (1). Although the naturally occurring or the most arailable forms of the substrate were preaumably used, the configurational prefixes D- and L- have been omitted from this text whenever the original article failed to provide specific information as to which was correct.

To discuss the culture conditions in detail would be beyond the scope of this review, and the main factors only will be oummarized briefly. The mutrient medium was usually a 2-30\% aqueous solution of the compound serving as the source of carbon. This solution contained also $0.5-2 \%$ of a source of inorganic nitrogen, smaller amounts of other inorganic salts, and posgibly an organic stimulant (44). The medium used in the production of kojic acid from xylose by A. flavus was (36): xylose, $150 \mathrm{~g} \cdot$; amonium nitrate, $1 \mathrm{~g} \cdot$; potassium dihydrogen phosphate, $0.625 \mathrm{~g} \cdot$; magnesium sulfate heptahydrate, $0.5 \mathrm{~g} \cdot ;$ and water, 1 liter. The sterilized solution was adjusted to a $\mathrm{pH}$ value varying from 2 to 6 , inoculated with the mold, and incubated at $15-38^{\circ} \mathrm{C}$. for a period between a few days and six weeks. The molds were generally grown on the surface of the medium where they were exposed to air, but submerged growths were also recomnended $(39,45)$. In the latter case the oxygen 
TABLE I

Carbon Sources for the Biosynthesis of Kojic Acid

\begin{tabular}{|c|c|c|}
\hline & Compound & References \\
\hline $\mathrm{c}_{2}$ & $\begin{array}{l}\text { Ethanol } \\
\text { Glycine } \\
\text { Sodium acetate }\end{array}$ & $\begin{array}{l}25,26 \\
27 \\
27\end{array}$ \\
\hline$c_{3}$ & $\begin{array}{l}\text { Dihydroxyacetone } \\
\text { Glyceraldehyde } \\
\text { Glycerol } \\
\text { Sodium lactate } \\
\text { Sodium pyruvate }\end{array}$ & $\begin{array}{l}28,29,30 \\
30 \\
6,7,20,25,29,31,32,33,34 \\
25 \\
25,27\end{array}$ \\
\hline$c_{4}$ & Tartaric acid & 33 \\
\hline$c_{5}$ & $\begin{array}{l}\text { Adonitol } \\
\text { Arabinose } \\
\text { Xylose }\end{array}$ & $\begin{array}{l}34 \\
20,31,32,33,35 \\
20,31,32,33,34,35,36\end{array}$ \\
\hline$c_{6}$ & $\begin{array}{l}\text { 2-Desoxyglucose } \\
\text { Dulcitol } \\
\text { Fructose } \\
\text { Galactose } \\
\text { Gluconic acid } \\
\text { Gluconolactone } \\
\text { Glucose } \\
\text { Inositol } \\
\text { Mannitol } \\
\text { Mannose } \\
\text { Sorbitol }\end{array}$ & $\begin{array}{l}26 \\
32,33,34 \\
5,20,24,25,31,32,33,34 \\
20,25,32,34 \\
32 \\
34 \\
4,5,6,20,25,28,30,31,32,33,34,35,37,38,39 \\
32,33 \\
6,20,24,33,34,40 \\
32,33,34 \\
32,34\end{array}$ \\
\hline$c_{7}$ & $\begin{array}{l}\text { Quinic acid } \\
\text { Shikimic acid }\end{array}$ & $\begin{array}{l}30 \\
30\end{array}$ \\
\hline$\left(c_{6}\right)_{2}$ & $\begin{array}{l}\text { Lactobionic acid } \\
\text { Lactose } \\
\text { Maltose } \\
\text { Sucrose } \\
\text { Trehalose }\end{array}$ & $\begin{array}{l}30 \\
20 \\
32,41 \\
5,6,20,31,32,34,40,42,43 \\
30\end{array}$ \\
\hline$\left(c_{6}\right)_{n}$ & $\begin{array}{l}\text { Imulin } \\
\text { Pectin } \\
\text { Starch }\end{array}$ & $\begin{array}{l}32 \\
30 \\
20\end{array}$ \\
\hline
\end{tabular}


requirements of the mold had to be satisfied by bubbling air through the medium, or by constant agitation. The articles by Birkinshaw and co-workers (20), May and co-workers (37), Kluyver and Perquin (39), Barham and Smits (36), and Barnard and Challenger (26) are readily available and contain detailed information about the factors influencing the production of kojlc acid by molds. A good summary of these factors was given by Foster (46).

The Isolation of Kojic Acid:- After the incubation period, the mycelium of the mold was removed, and the kojic acid produced was recovered from the culture filtrate by one of the following methods: precipitation as the copper salt $(3,26)$, extraction with ethyl acetate (7), contimuous extraction with ether $(28,37)$, evaporation to a small volume leading to crystallization (20), or crystallization at $0^{\circ} 0 .(21)$, and adsorption on active carbon followed by elution with butyl acetate which was saturated with dry ammonia (47). The last mentioned method was recommended for low concentrations of kojic acid. Phares and co-workers (48) recently worked out a method, based on partition chromatography, for the separation of kojic acid from any other organic acids formed in the biosynthesia. The reported yields of kojic acid ranged from traces to close to $70 \%$, but these figures were hardly comparable in many cases. The surface growth of the molds was too heterogeneous to be readily reproducible, particularly in long incubation periods. Kluyver and Perquin (39) devised a method for the standardization of growth conditions. Uniformly produced homogeneous mold substance was transferred to a fresh medium, and the changes brought about in it by the mold were evaluated after 24 hours of incubation. 
The Quantitative Estimation of Kojic Acid:- In solutions, the quantity of kojic acid has been determined colorimetrically by measurIng the intensity of the red color produced with ferric chloride $(31,49)$, and volumetrically, in the absence of other organic acids, by titration with standard dilute alkali, using alizarin orange $R$ (37) or phenolphthalein $(5,36)$ as an indicator. Other volumetric methods were based on the quantitative decomposition of kojic acid by aqueous alkaIine solutions of lodine according to equation $A(50)$, and on the rapid

$$
\begin{aligned}
& \mathrm{C}_{6} \mathrm{H}_{6} \mathrm{O}_{4}+5 \mathrm{I}_{2}+11 \mathrm{NaOH} \longrightarrow \\
& \longrightarrow \mathrm{CHI}_{3}+7 \mathrm{NaI}+6 \mathrm{H}_{2} \mathrm{O}+\mathrm{HCOONa}+(\mathrm{COONa})_{2}+\mathrm{HOCH}_{2} \mathrm{COONa}
\end{aligned}
$$<smiles>CC1(O)CC2CC1OC=CC2=O</smiles>

addition of four atoms of lodine to one molecule of kofic acid in a glacial acetic acid - benzene solution containing mercuric acetate as a catalyst, as shown in equation B (51).

Gravimetric methods involved the quantitative precipitation of kojic acid as the copper salt (37, 52), or careful extraction, drying and weighing of kojic acid proper (35).

The Mechanism of the Formation of Kojic Acid:- Speculation about the mode of formation of the $\gamma$-pyrone ring of kojic acid from the carbohydrates subjected to the biosynthetic process began practically with the discovery of the substance, and the theories tended to change 
with the conversion of each new type of compound to kojic acid. These theories are best considered in their chronological sequence.

Yabuta (3) connected the formation of kojic acid with the redox changes concerned in the reduction of hexoses to the corresponding alcohols. The conversion of mannitol (40) to kojic acid rendered his view less probable. Traetta Mosca (5) belleved the formation of kojic acid to be the first step in the alcoholic fermentation of sugars, but this tbeory was linked with his early misconception about the structure of kojlc acid. Traetta Mosca and Pret1 (7) later recognized it as a hydroxypyrone. They also obtained kojic acid from glycerol; this was the first instance of the formation of the acid from a compound with less than six carbon atoms. They assumed the initial conversion of glycerol to glyceraldehyde and the condensation of two molecules of the latter to give glucose, the known starting material in the production of kojic acid.

Kinoshita (40), then Haworth (53), noted the close structural relationship of kojic acid (XXIV) and glucose (XXII), and suggested that the latter was changed to the former through oxidation to a ketonic intermediate (XXIII), which then was dehydrated to give XXIV. This was frequently called the "carving out" theory of the formation of kofic acid. Opposed to it was the "fission" theory, which could be dated from the first conversion of pentoses $(31,32,35)$ to kojic acid.

Challenger and co-workers (35) had hoped to obtain pyromeconic acid (XXVI) from pentoses (XXV) with A. oryzae, by analogy with the

* The unconvential formulae for the carbohydrates and the consecutive numbering of the carbon atoms of kojic acid, adopted in this oection, serve to facilitate comparison. 
<smiles>OC[C@H]1O[C@H](O)[C@@H](O)[C@@H](O)[C@H]1O</smiles>

XXII

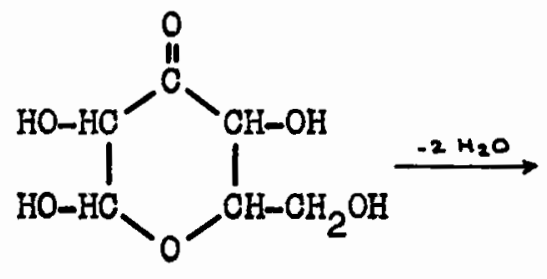

XXIII<smiles></smiles>

XXIV<smiles>O=c1ccoc(Oc2ccco2)c1O</smiles>

formation of kojic acid from hexoses. Instead, kojic acid was produced from L-arabinose and D-xylose. Corbellini and Gregorini (31), too, observed the production of kojic acid by A. flavus from those two pentoses and from D-fructose (XXVII); they noted that Haworth's scheme<smiles>C#CCc1occc(=O)c1O</smiles>

XXVII

XXVIII

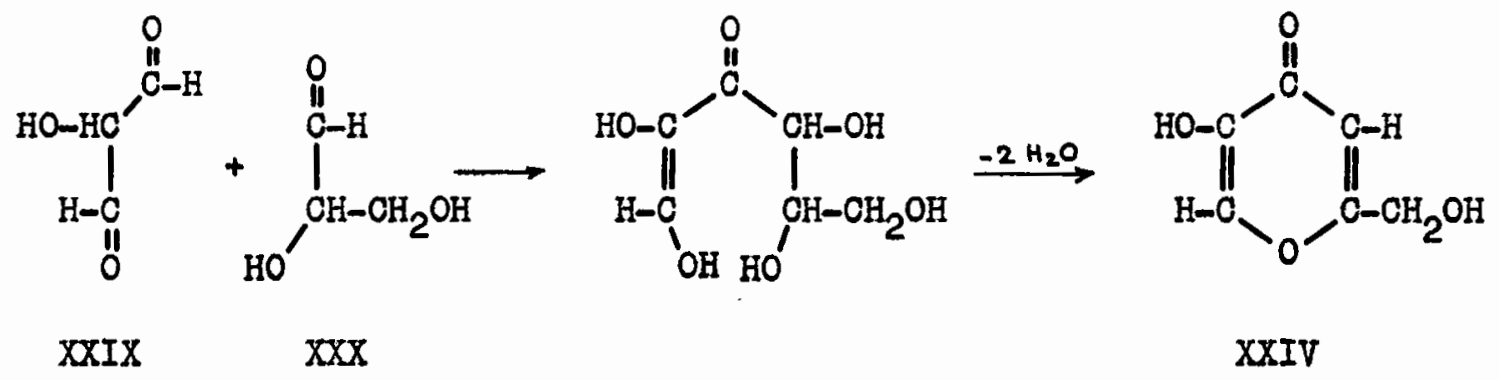

would require the formation of an isomer (XXVIII) of kojic acid from 
XXVII, and suggested that kojic acid (XXIV) was formed directly by the condensation of two molecules containing three carbon atoms, without the prior formation of a hexose. In their example they used glyceraldehyde $(\mathrm{XXX})$ and glyceric dialdehyde (XXIX) which could be produced from sugars by fission and from glycerol by oxidation. Their theory was rendered more probable by the great importance of trioses and triosephosphates in other metabolic processes, and was supported by the independent observation of Challenger and co-workers (29), that dihydroxyacetone, another oxidation product of glycerol, was converted to kojic acid in yields exceeding $30 \%$. These workers also succeeded in isolating dihydroxyacetone (or glyceraldehyde) in the form of its phenylosazone from cultures of A. niger on a medium containing $5 \%$ glycorol. They came to essentially the same conclusion as Corbellini and Gregorini (31), and thought it probable that the conversion of glucose into kojic acid might also involve ring fission and formation of a triose, rather than direct transformation. Others (37) began to favor this theory, despite Maurer's chemical synthesis of kojic acid from D-glucose (54).

All fungi capable of producing kojic acid were known to produce ethanol at the same time. Hence acetaldehyde would also have to be present in the media, and from this Birkinshaw and co-workers (20) reasoned that acetaldehyde, a diose, might be the building stone in the biosynthesis of kojic acid. This concept was favored by the discovery that kojic acid was produced by some acetic acid bacilli (24), since acetaldehyde was an intermediate in the oxidation of ethanol to acetic acid by these organisms. In an alternative theory advanced by Birkinshaw and co-workers (20), it was assumed that the fungi first anabolized 
whatever carbon source they had to a reserve carbohydrate, and later hydrolysed it to a hexose, which was then converted to kojic acid. Tamiye (33) reached the same conclusion, and considering the customary long culturing periods, it seemed entirely reasonable. Their view was shared later by Kluyver and Perquin (39), when these workers found that with short incubation periods in special replacement cultures, A. flevus produced practically no kojic acid in media containing pentoses, sugar alcohols, and hexoses other than glucose. Under identical conditions, production of kojic acid from glucose was at an optimum. The negative results in the other cases suggested that only glucose was converted directly to kojic acid, and all the other compounds were first assimilated into a reserve carbohydrate.

Trioses, particularly dihydroxyacetone, were again considered as the most important intermediates in the formation of kojic acid, when Katagiri and Kitahara (30), who worked with A. oryzae, reported a $55 \%$ yield of kojic acid from dihydroxyacetone, traces from glyceraldehyde, but none from acetaldehyde and diethyl acetal. Gould (34) found that the production of kojic acid from glucose by A. tamarii was not affected by the presence of aldehyde-trapping reagents, e.g. dimedone or bisulfite, in the growth medium; and that no kojic acld was produced by the fungus if mold substance, i.e. reserve carbohydrate, was the only source of carbon. Several theories were thus contradicted in one paper, but the extreme sensitivity of these microbiological processes to seemingly minor changes in conditions should be kept in mind. Gould (34) found further that the formation of kojic acid probably did not proceed through phosphoric esters, as it took place readily in phosphate-free media 
conteining glucose, xylose and gljcerol respectively.

Sakaguchi and co-workers (25) succoeded in producing kojic acid from ethanol by A. oryzae, and thereby provided the first experimental evidence supporting the diose intermediate theory. Barnard and Challenger (26) devoted considerable attention to this problem. They obtained $12-17 \%$ yields of kojic acid at $32^{\circ} \mathrm{C}$. from a culture of A. oryzee on a basal salt medium containing $1.3-2.1 \%$ of ethanol, but none at lower temperatures or higher ethanol concentrations. The carefully washed fungus was grown on the basal salt medium alone for six weeks, and its failure to produce kojic acid eliminated the possibility of kojic acid production from reserve carbohydrate in the experiments with ethenol. All cultures of the fungus on ethanol solutions were shown to contain acetaldehyde, and the addition of dimedone, but not of bisulfite, reduced the yield of kojic acid to $5 \%$ and delayed its formation. They saw further evidence for the "fission" theory and against the "carving out" theory in the fact that three derivatives of glucose which were not expected to undergo fission gave no $\gamma$-pyrone derivatives, and that a $20 \%$ yield of kojic acid was obtained from 2-desoxyglucose (XXXI), which by the "carving out" process should have given rise to 2-(hydroxgmethyl)$-\gamma$-pyrone (XXXII). Clearly, the accumulated infornation revealed many<smiles>O=C1CC(O)C(O)C(CO)O1</smiles>

$X X X I$<smiles></smiles>

XXXII

details, but the exact nature of the biosynthetic process remained a 
matter of conjecture.

Very recently, Arnstein and Bentley applied the isotopic tracer technique in their studies of kojic acid. First they investigated the mechanism of the alkaline cleavage and degradation of dimethyl kojic acid (XXXIII) (55). Yabuta (4) had found that treatment of XXXIII with barium hydroxide yielded equimolar quantities of formic acid, methoxyacetone (XXXIV) and methoxyacetic acid (XXXV). The same three compounds

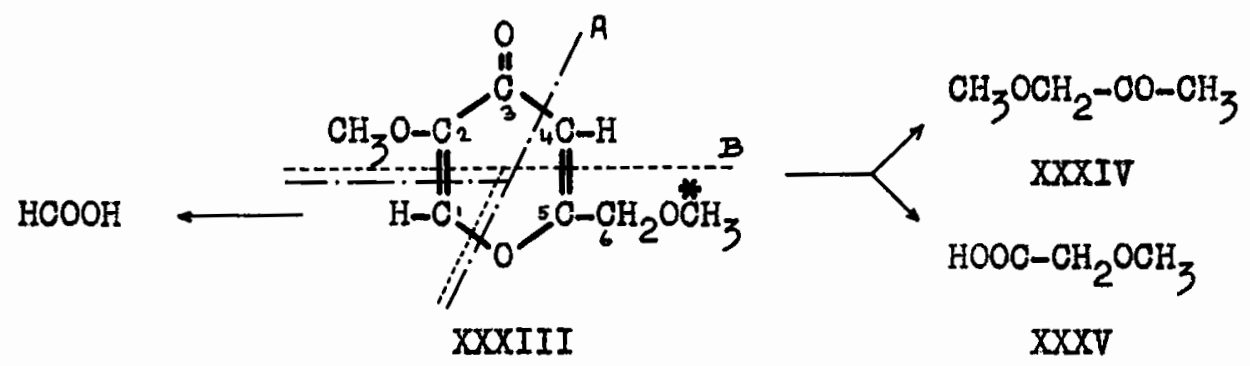

were obtained from XXXIII labelled with ${ }^{14} \mathrm{C}$ in one of the methyl groups (marked by *) (55); the formic acid was completely non-radioactive, and the whole radioactivity was distributed between the other two fragments, the share of XXXV being about $20 \%$ higher than that of XXXIV. This result indicated that the cleavage occurred in two different ways: according to scheme A (shown by the relevant dotted line in XXXIII), carbon atoms $C_{2}$ and $C_{3}$ gave XXXV, and $C_{4}, C_{5}$ and $C_{6}$ gave XXXIV, while according to scheme $B, C_{5}$ and $C_{6}$ appeared in XXXV and $C_{2}, C_{3}$ and $C_{4}$ in XXXIV (see footnote to $\mathrm{p} .12)$. The greater radioactivity of XXXV pointed to a slight predominance of mode B. Formic acid was formed in both cases from $c_{1}$.

Next, Arnstein and Bentley (28) produced kojic acid with A. oryzae and A. flavus-oryzae from media containing $1-(14 \mathrm{C})$-D-glucose, 
$3,4-\left({ }^{14} C_{2}\right)-D$-glucose and $2-\left({ }^{14} \mathrm{C}\right)-1,3$-dihydroxyacetone respectively. Between 2 and $20 \%$ of the ${ }^{14} \mathrm{C}$ was recovered in kojic acid. The kojic acid was converted to its ethyl methyl ether (XXXVI) in each case, and the degradation of this unsymmetrical ether by hot aqueous barium hydroxide, the separation of the fragments and their assay for radioactivity gave an exact measure of the amount of ${ }^{14} \mathrm{C}$ incorporated into each of the six carbon atoms of kojic acid. The scheme for this operation is given in Fig. 1 (see footnote to p. 12).

After treatment of XXXVI with aqueous barium hydroxide, the liquor contained formic, methoxyacetic and ethoxyacetic acids, methoxyacetone and ethoxyacetone, produced by the two modes of cleavage XXXVI A and XXXVI B. The formic acid was converted with red mercuric oxide to carbon dioxide; this was recovered as barium carbonate, the radioactivity of which was a measure of the ${ }^{14} \mathrm{C}$ incorporated into $c_{1}$ of kojic acid in the biosynthetic process. The alkoxyacetones were removed from the liquor by steam distillation, and converted to lodoform and a mixture of methoxy- and ethoxyacetic acids. The lodoform was recovered by filtration; its radioactivity indicated the proportion of ${ }^{14} \mathrm{C}$ incorporated into $C_{4}$. The alkoxyacetic acids were isolated by continuous extraction of the filtrate with ether, and those in the original liquor were recovered in the same way. These acids were separated chromatographically and converted to their silver salts. Methoxyacetic acid from both sources contained $C_{5}$ and $C_{6}$ of kojic acid, while all of the ethoxyacetic acid was composed of $\mathrm{C}_{2}$ and $\mathrm{C}_{3}$. Assay of the silver salts for radioactivity therefore gave the amounts of ${ }^{14} \mathrm{C}$ incorporated in $\mathrm{C}_{2}+\mathrm{C}_{3}$ and in $c_{5}+c_{6}$ respectively. Degradation of the silver salts with bromine 


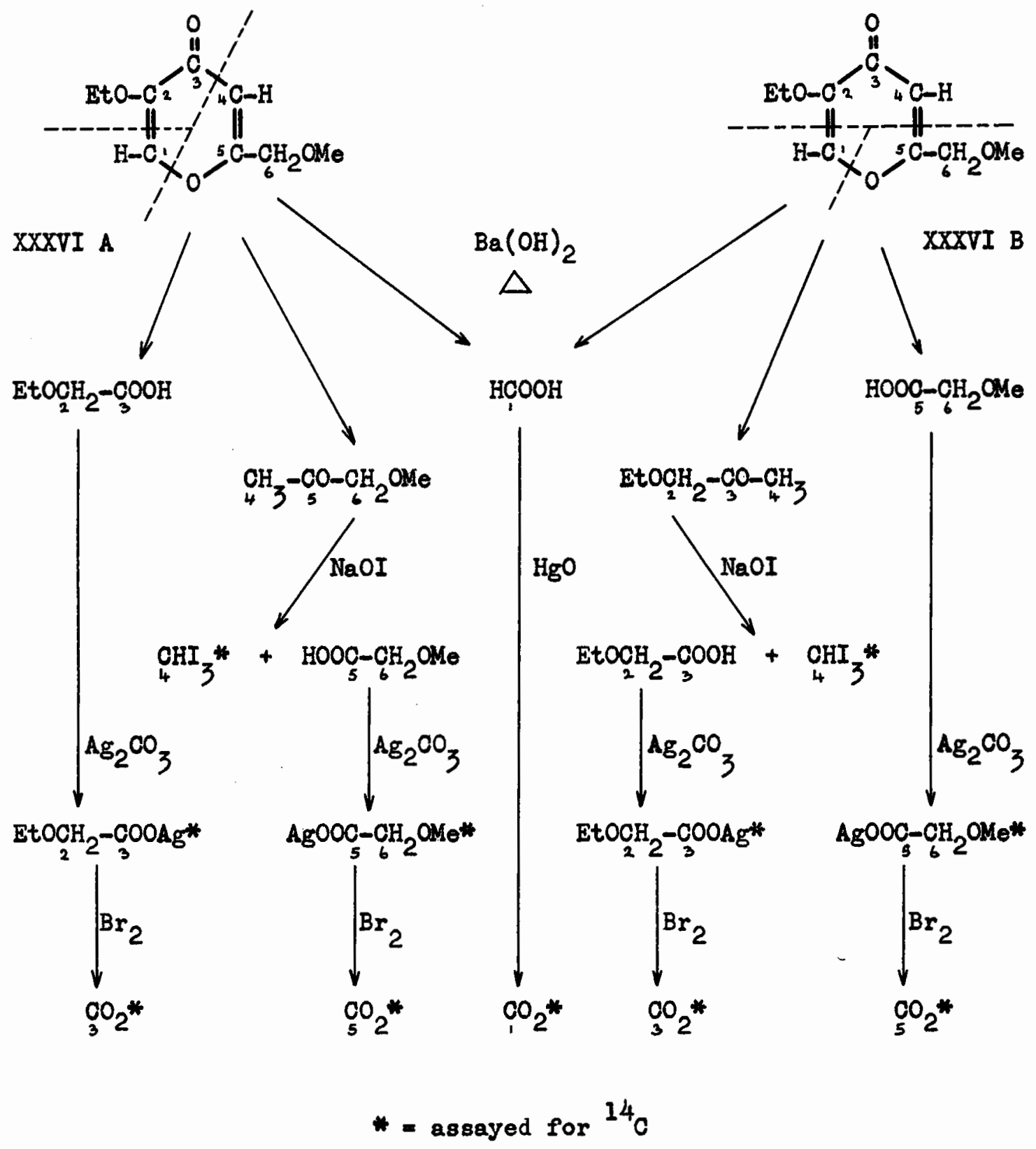

Fig. 1:- Scheme for the Degradation of the Ethyl Mothyl Ether of Kojic Acid, and the Separation and ${ }^{14} \mathrm{C}$ Assay of the Fragments. 
liberated carbon dioxide containing $\mathrm{C}_{3}$ from ethoxyacetic acid, and carbon dioxide containing $\mathrm{C}_{5}$ from methoxyacetic acid. These portions of carbon dioxide were also recovered and assayed in the form of barium carbonate. The amount of ${ }^{14} \mathrm{C}$ which was incorporated into $\mathrm{C}_{2}$ and $\mathrm{C}_{6}$ was finally calculated by difference.

The results of these experiments showed that in kojic acid produced from 1-( $\left.{ }^{14} \mathrm{C}\right)$-D-glucose, $70-90 \%$ of the ${ }^{14} \mathrm{C}$ was located in $C_{1}$ and 6-16\% in $C_{6}$; in kojic acid produced from 3,4-( $\left.{ }^{14} C_{2}\right)$-D-glucose, $90 \%$ was located in $\mathrm{C}_{3}$ and $\mathrm{C}_{4}$; and in kojlc acid produced from 2-( $\left.{ }^{14} \mathrm{C}\right)-1,3-\mathrm{di}-$ hydroxyacetone $60-70 \%$ was to be found in $C_{2}$ and $C_{5}$ of kojic acld. These distributions of radioactivity indicated very definitely that kojic acid was formed from D-glucose largely by direct conversion, and no splitting of the carbon chain occurred. The distribution of ${ }^{14} \mathrm{C}$ in the kojic acid produced from labelled dihydroxyacetone, moreover, showed clearly that if free dihydroxyacetone were an important intermediate, the conversion of $1-\left({ }^{14} C\right)-D-g l u c o s e$ would have led to a more extensive incorporation of the ${ }^{14} \mathrm{C}$ into atoms $\mathrm{C}_{2}-\mathrm{C}_{6}$ of kojic acid. The presence of $6-16 \%$ of the ${ }^{14} \mathrm{C}$ in $\mathrm{C}_{6}$ nevertheless pointed to a minor pathway in the formation of kojic acid, which involved the splitting of the glucose molecule, and the recombination of the trioses thus formed.

Arnstein and Bentley believed that the direct conversion of glucose (XXII) might proceed by the following sequence: enzymatic oxidation of XXII to gluconolactone (XXXVII), further oxidation of XXXVII to 3-ketogluconolactone (XXXVIII), and conversion of XXXVIII by dehydration and enzymatic reduction to kojic acid (XXIV). The arguments in favor of this mechanism include the production of gluconic acid by a strain of 
<smiles>[R10]C#CC(=O)OC1OC(=O)C(O)C(=O)C1O</smiles>

A. oryzae (38), the frequent occurrence of gluconic and ketogluconic acids in fungi, and the utilization of gluconolactone (34) and gluconic acid (32) as carbon sources for the biosynthesis of kojic acid.

In further studies, Arnstein and Bentley (56) demonstrated the presence of aldolase and triosephosphete isomerase in fung 1 producing kojic acid. They also found that both production and destruction of kojic acid was rapid in media with high phosphate levels, and slow at lower phosphate levels. They preferred to consider kojic acid as a normal metabolite of the fungi, rather than as an end product.

Lastly, they (27) investigated the incorporation of small molecules into kojic acid. Buffered solutions of pyruvic acid, acetic acid, glycine, and some other similar compounds, all labelled with ${ }^{14} \mathrm{C}$, were added to Aspergillus cultures grown initially on unlabelled glucose. The recovery of ${ }^{14} \mathrm{C}$ in kojic acid was usually $108 \mathrm{~s}$ than $1 \%$, and the labelled carbon atoms were distributed over the whole molecule, predominating, however, in all cases in $\mathrm{C}_{4}, \mathrm{C}_{5}$ and $\mathrm{C}_{6} . \mathrm{A}$ similar predominance of the radioactivity in the "lower" half of the kojic acid molecule was also observed in the work with labelled dihydroxyacetone in the presence of non-radioactive glucose (28), and might be explained (27) in both instances by the condensation of a labelled three carbon 
intermediate, e.g. pyruvic acid or dihydroxyacetone, with an unlabelled triose or triosephosphate, derived from glucose. In neither case were major quantities of glucose converted to kojic acid by this pathway. These studies are of great significance, and give for the first time a clear picture of some importent phases of the biosynthesis of kojic acid; but far from providing a quick solution to all the problems, they also fully reveal the great complexity of the conversion of carbohydrates to a $\gamma$-pyrone, even one as relatively simple as kojic acid.

Chemical Synthesis

The otarting materials for the chemical synthesia of kojlc acid were also carbohydrates, but only hexoses and their derivatives were used for this purpose. Maurer and his co-workers studied the synthesis extensively in order to clarify the mode of formation of the $\gamma$-pyrone system from carbohydrates. They succeeded in preparing diacetylkojic acid (XlIII) by purely chemical means, first from D-glucose (54) and then from D-galactose (57). The mechanism of formation of XIIII, which did not contain any asymmetrical carbon atoms, was apparently not dependent on the configuration of the hexose. The essential steps of the synthesis are given briefly in the following sequences the elements of hydrogen bromide were removed from tetra-0-acetyl-D-glucopyranosyl bromide (XXXIX) by diethylamine in benzene, the resulting crystalline tetra-Q-acetyl-2-hydroxy-D-glucal (XI) was chlorinated in dry ether, the simupy mixture of isomeric dichlorides (XII) was hydrolysed by moist silver carbonate or water to $2,3,4,6$-tetra-0-acetyl-D-glucosone hydrate (XIII), and XIII was finally converted to XIIII by acetic anhydride and 


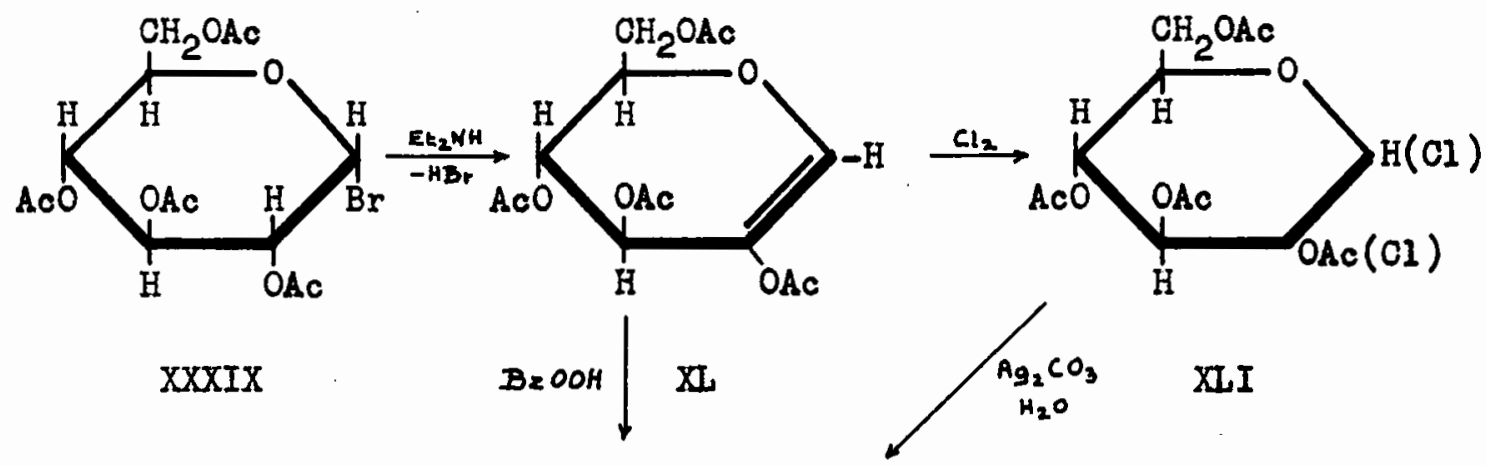

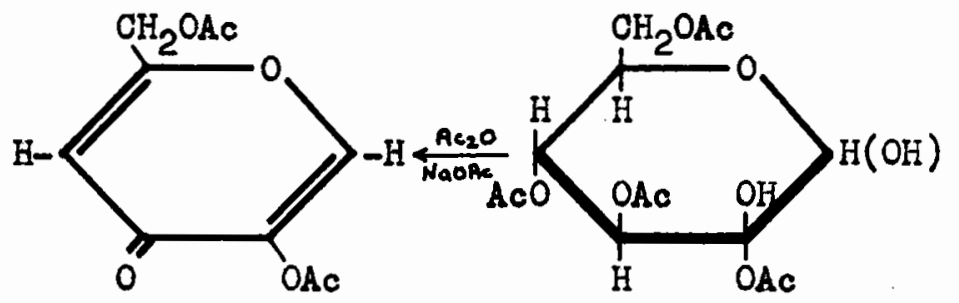

$X \amalg I I I$

$X \amalg I I$

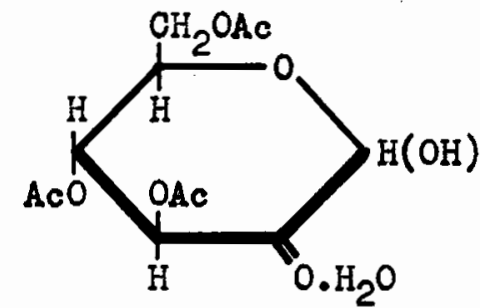

XIIV

pyridine at $0^{\circ}$ C., or by heating with acetic anhydride and anhydrous sodium acetate. In this last step, XIII lost two molecules of acetic acid and one molecule of water in the formation of the $\gamma$-pyrone ring. When sodium bicarbonate was substituted (58) for silver carbonate, XII was hydrolysed to $3,4,6$-tri-0-acetyl-D-glucosone hydrate (XIIV). Both XIIV and its anhydrous form were converted to XLIII by acetic anhydride and pyridine, or slowly even by $2 \%$ aqueous pyridine. The best yield of XLIII was obtained from XII via XIII $(58,59)$. The study was extended to other derivatives of D-glucosone (60); 1-0-benzoyl-3,4,6-tri-0-acetyl-D-glucosone and 1-0-acetyl-3,4,6-tr1-0-benzoyl-D-glucosone were both stable to pyridine, apparently because the acyl group at position 1 prevented their transformation to kojic acid derivatives. The chlorination of a tetrabenzoyl analog of $\mathrm{XI}$, and hydrolysis of the dichloride, yielded 2,3,4,6-tetra-0-benzoyl-D-glucosone (XIV). This compound had an 


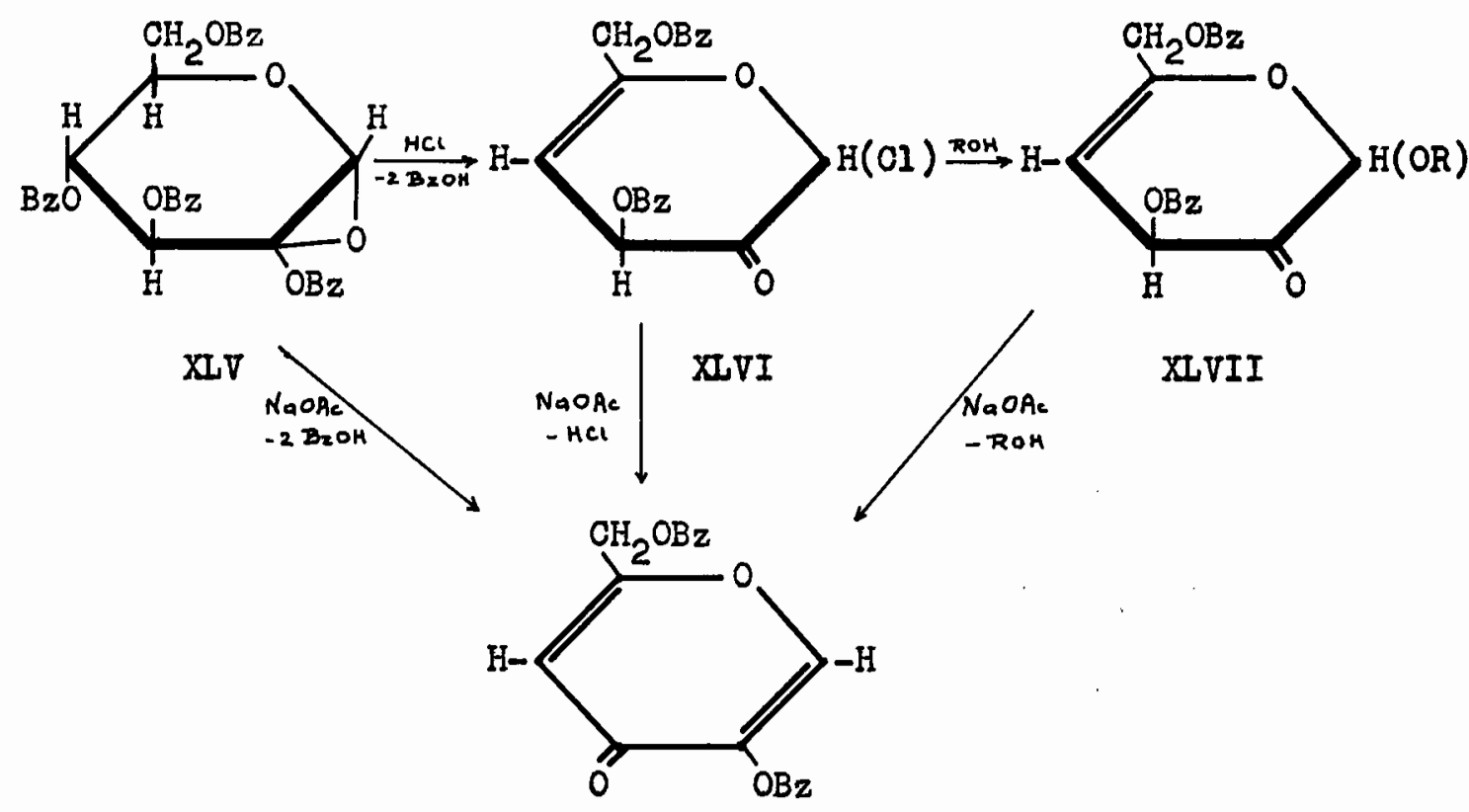

XIVIII

unusual 1,2-ethylene oxide structure, and no acyl group at position 1. The treatment of $\mathrm{XLV}$ with pyridine, or sodium acetate, resulted in its quantitative conversion to dibenzoylkojic acid. (XIVIII). Dry hydrogen halides cleaved the ethylene oxide ring of XIV (6I) and a halogen atom entered position 1. At the same time, two molecules of benzoic acid were removed from XLV, with creation of a carbonyl group at position 2 and of a carbon-to-carbon double bond between positions 4 and 5 . Dry hydrogen chloride in benzene thus converted XIV to 3,6-di-0-benzoyl-D-glucoson-4,5-enyl chloride (XIVI). When XIVI was warmed in alcohols, the ohlorine atom was easily replaced by various alkoxy groups to give glycosides (XLVII). Like XIV, both XLVI and XIVII were converted to XIVIII by sodium acetate (61).

From these results, Maurer concluded (58) that the carbonyl group of position 2 in all these glucosone derivatives, as represented by the 
simplified formula XLIX, was shifted by the action of pyridine or sodium acetate via a common enediol intermediate (L) to position 3 (LI), and the molecule was finally stabilized by the expulsion of the appropriate small molecules (water, acetic acid, etc.) and the formation of the $\gamma$-pyrone ring (LII). This process was accompanied by the migration

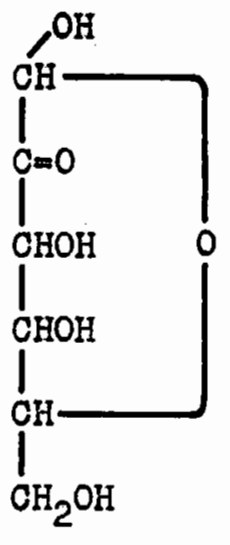

XIIX

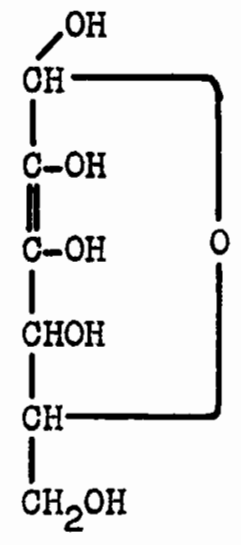

L

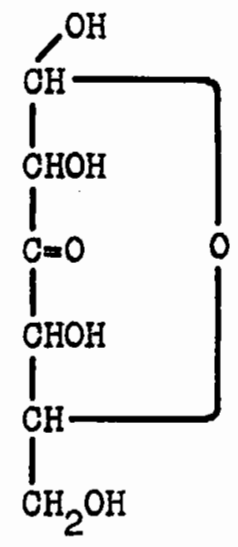

LI

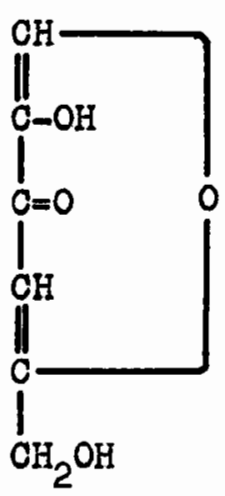

LII

of an acyl group from position 3 to position 2. The creation of a double bond between positions 4 and 5 of XVI was thought to be a preliminary stage, which was stabilized as the $\gamma$-pyrone ring (61) by changing to the conjugated system of XIVIII. This transformation proceeded in a sequence analogous to that leading from XIIX to LII.

Maurer (62) investigated briefly the ability of the reducing unit of disaccharides to form a $\gamma$-pyrone mucleus without cleavage of the glycosidic linkage. Hepta-0-acetyl-2-hydroxycellobial (LIII) and hepta-0-acetyl-2-hydroxygentiobial (LIV) were each subjected to the series of reactions which led from XI to XIIII. The final products were not isolated; but, after being treated briefly with alkali, the solution of the product from LIV gave a wine color with ferric chloride. This test 


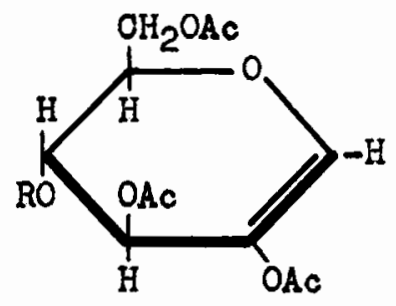

LIII

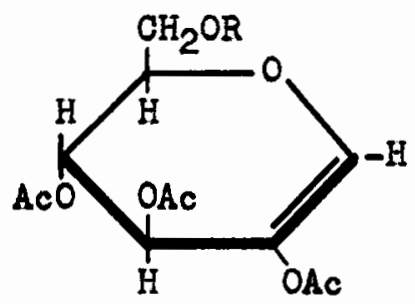

LIV

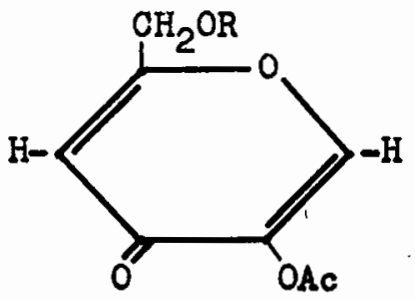

LV

$R=($ Tetra-0-acetyl-D-glucopyranosyl)

indicated the formation of a hydroxy- $\gamma$-pyrone, presumably a glycoside of acetylkojic acid (LV). No such color test was given by the product from LIII. Presumably the glucosyl residue, when attached to carbon 4, as in LIII, interfered with the deacetylation leading to the formation of the $\gamma$-pyrone nucleus. When it was attached to carbon 6,28 in LIV, the transformation was not hindered, because carbon 6 did not become part of the $\gamma$-pyrone mucleus.

The synthesis of kojic acid was simplified by Stacey and Turton (63), when they succeeded in converting XI directly to XIII with perbenzoic acid, thus eliminating the chlorination step. The mechanism of the formation of kojic acid by chemical synthesis was interpreted by them, and by Isbell, in terms of consecutive electron displacement. According to IsbelI (64), acetic anhydride and sodium acetate enolized keto acetatea in the way that bases and amphoteric solvents promote ordinary enolizations. In addition to favoring enolization, these substances, which were capable of supplying and accepting acetate ions, also caused the deacetylation of XIII. Isbell visualized the migration of the acetyl group from position 3 to position 2 by means of an intermediate complex of the orthoester type (LVI) with elimination of a proton and an acetate 


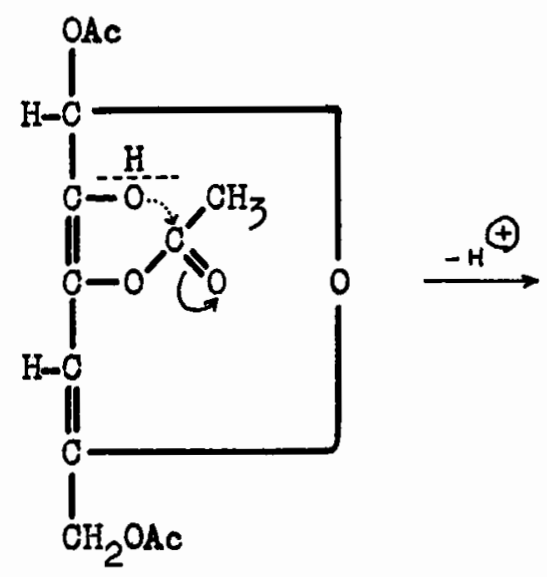

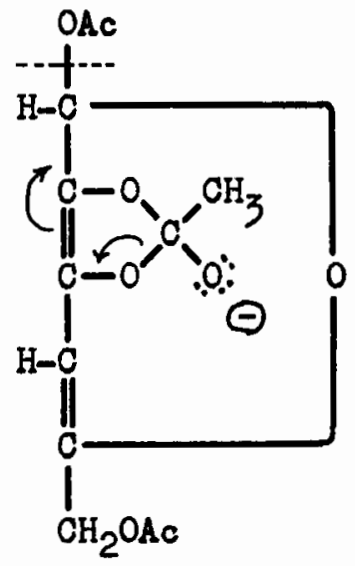

LVI

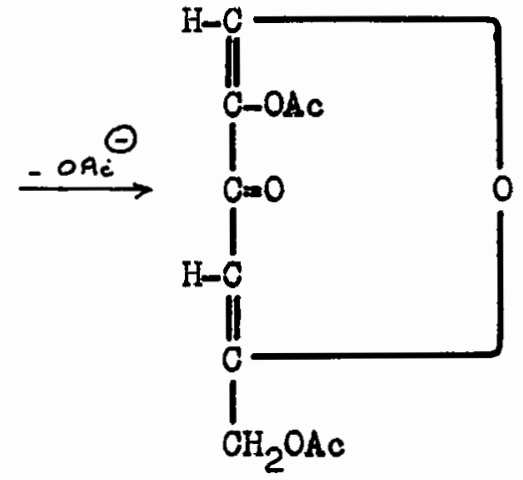

XIII

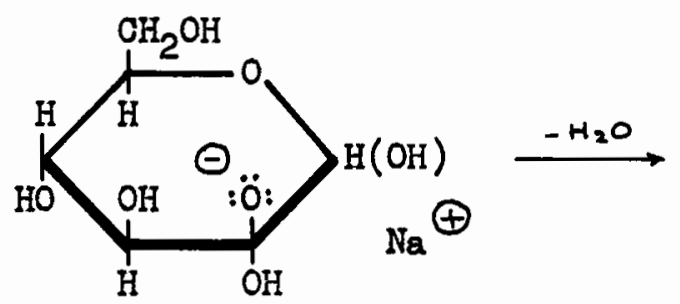

LVII

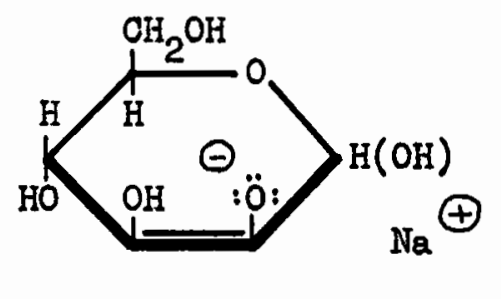

LVIII

ion. Stacey and Turton (63) found from ultraviolet absorption data that boiling dilute alkali, too, converted XII to kojlc acid (II), and consequently they considered the assumption of the ortho-acetate structures unnecessary. They suggested a mechanism for the transformation of XIII by alkali which was essentially the same, in its initial stages, as that suggested by Isbell (64). The aqueous alkall deacetylated XIII and neutralized the incipiently ionic hydrogen atom at position 2 (LVII). By loss of water between $C_{2}$ and $C_{3}$ an enediol (LVIII) was formed, which was then converted to kojic acid (II) in a manner and for reasons already outlined by Maurer (58). Stacey and Turton regarded a proton acceptor as one of the essential factors in these transformations. These investigations shed considerable light on the synthesis of 
the $\gamma$-pyrone ring; however, it is unlikely that any conclusions regarding the biosynthetic process could be drawn from them.

\section{Properties}

\section{Chemical Properties}

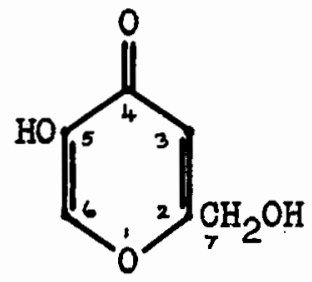

II
Kojic acid is a $\gamma$-pyrone. The conventional way of numbering the atoms in the $\gamma$-pyrone ring of kojic acid is show in structure II. For conrenience in the following discussion, however, the carbon atom in the hydroxymethyl side chain has been denoted as position 7. The hydroxyl

group in position 5 has a phenolic character (65), and the one in the side chain behaves like a primary alcohol whose reactivity is somewhat enhanced by the adjacent oxygen atom in the mucleus. Positions 3 and 6 heve very unequal reactivities. The common way of symbolizing the mucleus Indicates two carbon-to-carbon double bonds and a carbonyl group for kojic acid; however, they do not react in the manner typical of auch functions. This phenomenon is still most conveniently explained in terms of the resonence theory (66).

The chemical reactions and derivatives of kojic acid will be grouped according to these centers of its reactivity.

The Hydroxyl Groups:- The phenolic hydroxyl group of position 5 gives kojic acid its weakly acidic character $(3,4)$, and enables it to form salts with a muber of metals. No kojates of monovalent metals have 
as yet been prepared; sodium kojate is repeatedly mentioned in the 11terature, but the term apparently always refers to an aqueous solution of kojic acid neutralized with sodium hydroxide. Such solutions have frequently been used in bacteriological studies. Kojates of barium, calcium and strontium were reported by Yabuta (4), and the kojates of the divalent forms of the transition elements cobalt, copper, nickel, iron and mangenese were prepared by Wiley and his co-workers (67), who measured their magnetic susceptibilities. Wiley represented these salts as chelate complexes (LIX). The first three contained half a molecule of water of crystallization. The copper complex had been known earlier;

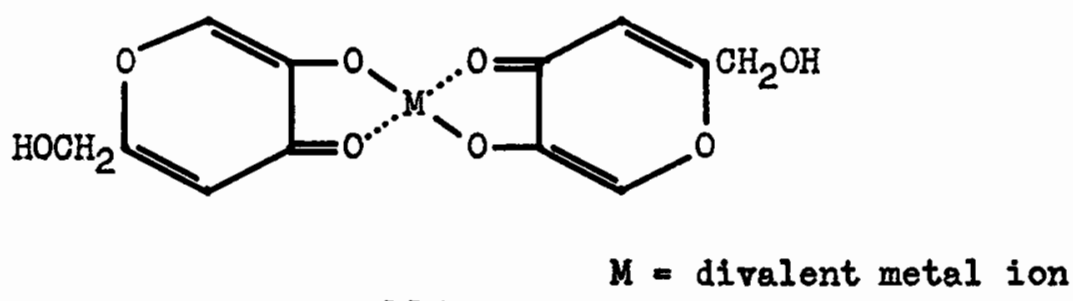

LIX

it played an important part in the isolation and estimation of kojic acid. The kojates of cadmium, lead, zinc, aluminum, lanthanum, neodymium, praseodymium and samarium were prepared by Musante (68), who also found kojic acid to be useful for the gravimetric determination of copper in the presence of several other ions. Methylemmonium kojate was prepared by Armit and Nolan (10).

The complex of kojic acid with ferric chloride, also formed through the phenolic hydroxyl group, gives rise to a characteristic wine color which persists even at a dilution of $1: 200,000$ (3). This colour resction permits the estimation of kojic acid by colorimetry; conversely, Moss 
and Mellon (69) based a method for the quantitative determination of ferric iron on this property of kojic acid. Although not ideal for this purpose, kojic acid is free from some of the interferences encountered with other reagents, its sensitivity is adequate over a wide range of iron concentrations, and the color produced is relatively stable. More data on this method have been provided by Mehlig and Shepherd (70). Many of the derivatives typical of hydroxy compounds have been prepared from kojic acid. The known ethers and esters aro collected in Table II. Reagents which are specific for phenolic hydroxyl groups, e.g. diazomethane $(7,8,10)$, allyl and phenacyl bromides $(71)$, usually formed 5-mono-0-derivatives. Triphenylmethyl chloride (72), on the other hand, reacted only with the primary alcoholic hydroxyl group, and the eaterification of kojic acid with caproic acid (71) also affected only position 7. Reagents which are non-specific tended to form 5-mono-0-derivatives if applied in equivalent quantities, and to react with both hydroxyl groups if applied in excess. Thus the 5-monomethyl ether of kojic acid was most conveniently prepared with one mole of dimethyl sulfate in aqueous alkali $(16,75)$, while with two moles the 5,7-dimethyl ether was formed (4). Similarly, the benzoylation of kojic acid with benzoyl chloride in ether gave 5-0-benzoylkojic acid (4), but with benzoyl chloride in the presence of aqueous alkali (4) or pyridine (5, 54) the 5,7-dibenzoate was produced. Acetic anhydride $(4,5,54)$ and phenylisocyanate (4) esterified both hydroxyl groups, and so did methanesulfonyl chloride at $-5^{\circ} \mathrm{C} .(76)$.

Very few of these derivatives were hydrolysed. Kojic acid was obtained by Maurer (54) by amonolysis of 5,7-di-0-acetylkojic acid. By 
Functional Derivatives of Kojic Aoid (a)<smiles>O=c1ccocc1</smiles>

\begin{tabular}{|c|c|c|c|}
\hline $\mathbf{R}$ & $\mathrm{R}^{\prime}$ & $\begin{array}{l}\text { Melting } \\
\text { point, }{ }^{\circ} \mathrm{C} .\end{array}$ & References \\
\hline $\begin{array}{l}\mathrm{H} \\
\mathrm{H} \\
\mathrm{H} \\
\text { Allyl } \\
\text { Benzoyl } \\
\text { 2,4-Dinitrophenyl } \\
\text { Ethyl } \\
\text { B -D-Glucopyranosyl } \\
\text { Methyl } \\
\text { Phenacyl } \\
\text { Acetyl } \\
\text { Benzoyl } \\
\text { Benzoyl } \\
\text { Ethyl } \\
\text { Methanesulfonyl } \\
\text { Methyl } \\
\text { Methyl } \\
\text { Phenacyl } \\
\text { Phenylcarbamyl }\end{array}$ & $\begin{array}{l}\text { Acotyl } \\
\text { Oaproyl } \\
\text { Triphenylmethyl } \\
\text { H } \\
\text { H } \\
\text { H } \\
\text { H } \\
\text { H } \\
\text { H } \\
\text { H } \\
\text { Acetyl } \\
\text { Benzoyl } \\
\text { Trlphenylmethyl } \\
\text { Methyl } \\
\text { Methanosulfonyl } \\
\text { Acetyl } \\
\text { Methyl } \\
\text { Acetyl } \\
\text { Phenyl carbamyl }\end{array}$ & $\begin{array}{l}133.5,136 \\
66.5-67 \\
185-185.5 \\
125-125.5 \\
135-136,135 \\
172-173 \\
110 \\
201 \\
161,165 \\
144.5-145 \\
102 \\
134-135,136 \\
206-208 \\
45-46 \\
109-110 \\
120-122 \\
90 \\
116-116.5 \\
170\end{array}$ & $\begin{array}{l}54,71 \\
71 \\
72 \\
71 \\
4,72 \\
73 \\
8 \\
74 \\
7,9,10,31,75 \\
71 \\
4,5,6,21,54 \\
4,5,31,54 \\
72 \\
28 \\
76 \\
71 \\
4,10 \\
71 \\
4\end{array}$ \\
\hline
\end{tabular}


interrupting the reaction at an early stage, he isolated 7-0-acetylkojic acid. The latter compound was also prepared by flurd and Sims (71) by treating 5,7-di-0-acetylkojic acid with anhydrous aluminum chloride at $110^{\circ}$ C. Yabuta and Anno (72) detritylated 5-benzoyl-7-tritylkojic acid with acetic acid.

Derivatives of kojic acid which have been obtained by exchange of other groups for the hydroxyl group of position 7 are listed in Table III. This hydroxyl group can be replaced readily by chlorine. Thus, 2-(chloromethyl)-5-hydroxy- $\gamma$-pyrone $(8,76,77)$ and 2-(chloromethyl)-5-methoxy-8'-pyrone $(8,75,79)$ were prepered with thionyl chloride from kojic acid and its 5-monomethyl ether, respectively; and 5-methanesulfonoxy-2-(methenesulfonoxymethyl)- $\gamma$-pyrone (LX) was changed to<smiles>[Y]C[R19](=O)O[Na]</smiles>
LXII

2-(chloromethyl)-5-methanesulfonoxy- $\gamma$-pyrone (LXI) with concentrated hydrochloric acid (76). Several other derivatives then became available through replacement of the reactive chlorine atom by other atoms or 
Functional Derivatives of Kojic Acid (b)<smiles>[R17]c1cc(=O)cco1</smiles>

\begin{tabular}{|c|c|c|c|}
\hline $\mathbf{R}$ & $\mathrm{R}^{\prime}$ & $\begin{array}{l}\text { Melting } \\
\text { point }{ }^{\circ} \mathrm{C} .\end{array}$ & References \\
\hline $\begin{array}{l}\mathrm{H} \\
\mathrm{H} \\
\mathrm{H} \\
\mathrm{H} \\
\mathrm{H} \\
\mathrm{H} \\
\mathrm{H} \\
\text { Benzoyl } \\
\text { Benzoyl } \\
\text { Methanesulfonyl } \\
\text { Methanesulfonyl } \\
\text { Methanesulfonyl } \\
\text { Methanesulfonyl } \\
\text { Methyl } \\
\text { Methyl } \\
\text { Methyl } \\
\text { Methyl } \\
\text { Methyl } \\
\text { Methyl } \\
\text { Phenylcarbamyl }\end{array}$ & $\begin{array}{l}\text { H } \\
\text { gec-Butylthio } \\
\text { Chloro } \\
\text { Iodo } \\
\text { Isothiouronioa } \\
\text { Thiocyanato } \\
\text { Trimethylammonio a } \\
\text { H } \\
\text { Chloro } \\
\text { H } \\
\text { Chloro } \\
\text { Pyridinio a } \\
\text { Pyridinio } \\
\text { H } \\
\text { Chloro } \\
\text { Diethylamino } \\
\text { Iodo } \\
\text { Triethylammonio a } \\
\text { Trimethylammonio a } \\
\text { H }\end{array}$ & $\begin{array}{l}166,151 \\
93 \\
163,166-167 \\
\text { dec. } \\
155 \\
- \\
229 \text { dec. } \\
128-129 \\
117-118 \\
99.5-101 \\
83.5-85 \\
192 \text { dec. } \\
190 \text { dec. } \\
70-71 \\
119-121 \\
41 \\
135-137 \\
72-74 \\
209 \text { dec. } \\
186-188\end{array}$ & $\begin{array}{l}8,76 \\
77 \\
8,31,76,77 \\
8 \\
77 \\
78 \\
79 \\
8 \\
8 \\
76 \\
76 \\
76 \\
76 \\
8,75 \\
8,75,79 \\
79 \\
8 \\
75 \\
75 \\
8\end{array}$ \\
\hline
\end{tabular}

a Quaternary chlorido

b Quaternary methanesulfonate 
groups. For instance, treatment with diethylamine in benzene introduced the diethylamino group at position 7 (79); with sec-butyl mercaptan and sodium in toluene, the sec-butylthio group (77); and with potassium thiocyanate in $95 \%$ ethanol, the thiocyanato group (78). Attempts to replace chlorine by the cyano group $(10,75)$, and to prepare Grignard reagents from 2-chloromethyl derivatives of kojic acid (75), failed. Removal of chlorine by reduction with tin in hydrochloric acid (75) and with zinc in glacial acetic acid $(8,76)$ produced various 2-methyl analogs of kojic acid. These compounds were also named as derivatives of allomaltol (VI), a substance discovered by Yabuta (8) during his study of the structure of kojic acid.

Some chloromethyl derivatives of kojic acid form quaternary salts with tertiary aliphatic amines $(75,79)$ and with thiourea (77). Quaternary pyridinium salts (LXII) were obtained by Looker and Okamoto (76) from LX and LXI in dry pyridine. Water-insoluble derivatives of kojic acid could be rendered water-soluble by these quaternizations. The Unoccupied Nuclear Positions:- Either of the hydrogen atoms in the unsubstituted positions 3 and 6 of the nucleus of kojic acid should be replaceable by other groups, but with a single exception, all the substitution reactions studied were restricted to position 6 . Originally this restriction was explained by assuming the existence of a tautomeric form of kojic acid (V) with a reactive methylene group at position 6. According to modern concepts, the phenolic hydroxyl group is beliered to activate the positions ortho and para to it. In the case of kojic acid, of the three positions in question, anly the one ortho position, namely position 6, is available for substitution. This 
activating effect of the phenolic function would be particularly marked in alkaline media, where kojic acid probably exists in the form of a phenoxide ion (LXIII); the negative charge would be shared by position 6 (LXIV), where it would create a center of attack for electrophilic<smiles>O=c1cc(O)occ1O</smiles>

LXIII<smiles>O=C1C=CC2(O)COC1C2=O</smiles>

LXIV

reagents. This is, of course, an oversimplification of the case, as doubtless both nuclear oxygen atoms have a considerable effect on the reactivity of the free nuclear positions; but it serves to rationalize the tendency of the substitution reactions.

Yabuta (4) prepared 6-phenylazokojic acid (LXV) using phenyldiazonium acetate. Quilico and Musante (80) studied the coupling reaction<smiles></smiles>

LXV<smiles>CCCNC1CC2CCCC(O1)C2(O)O</smiles>

LXVI<smiles></smiles>

LXVII, $\mathrm{R}=\mathrm{Cl}, \mathrm{NO}_{2}$<smiles>O=c1cc(-c2ccccc2)oc2ccccc12</smiles>

LXVIII 
between diazonium chlorides and kojic acid in greater detail. In aqueous alkali, kojic acid and diazotized o-toluidine, p-toluidine and p-bromoaniline yielded products which were analogous to LXV, but the product from the coupling of the phenyldiazonium salt with kojic acid was anomalous. The composition of this product included an additional molecule of water, and, quite unlike the others, it did not react with diazomethane to form a methyl ether. They concluded that 6-phenylazokojic acid was best represented as a hydrated form (LXVI) of a tautomer of LXV. Ettel and Hebký (79) also obtained LXV as a monohydrate, but they succeeded in methylating it with diazomethane. The coupling of aromatic diazonium sulfates with kojic acid in alcohol, or acetic acid, proceeded with the elimination of nitrogen, and the products were 6-arylkojic acids (LXVII) (80). This interesting reaction might serve as the first step in the synthesis of flavonols (LXVIII) from kojic acid. Kojic acid was brominated at position 6 with bromine water (4); the 2-chloromethyl derivative of kojic acid was brominated (81) with N-bromosuccinimide, and chlorinated (82) with sulfuryl chlorido and aluminum chloride, in both cases probably at position 6. C-benzoylation (83) and C-acetylation (84) of kojic acid, likewise at position 6, was accomplished by Woods, using a modification of the Friedel-Crafts reaction. The 2-carboxyethyl sidechain was introduced into position 6 in a reaction with $\beta$-bromopropionic acid ( 85 ).

The 5-allyl ether of kojic acid (LXIX) was rearranged by alcoholic hydrogen chloride at $60^{\circ}$ o. to 6 -allylkofic acid (LXX)(71). Such a migration of the allyl group from the oxygen atom to the adjacent ring position is characteristic of allyl aryl ethers, but with the latter 
compounds it usually occurs only at much higher temperatures.

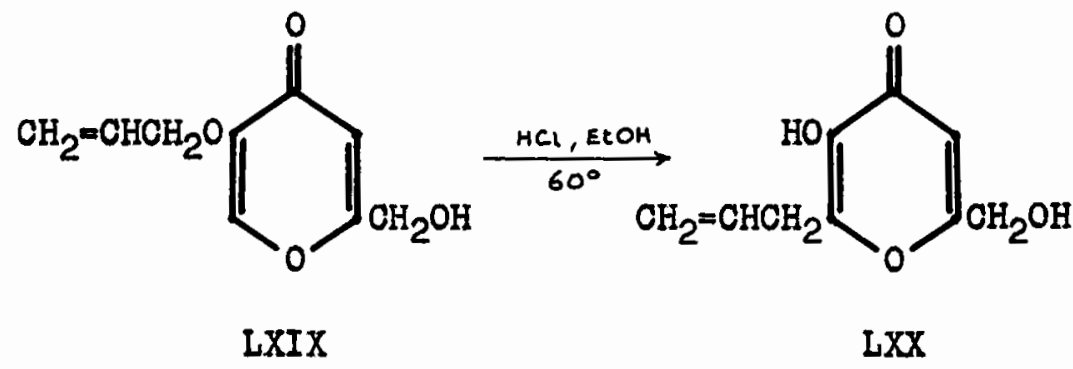

Another series of nuclear substitution reactions of kojic acid might be represented as the addition of kojic acid to reactive double bonds, following the familiar schemes A, B and C. These reactions, all

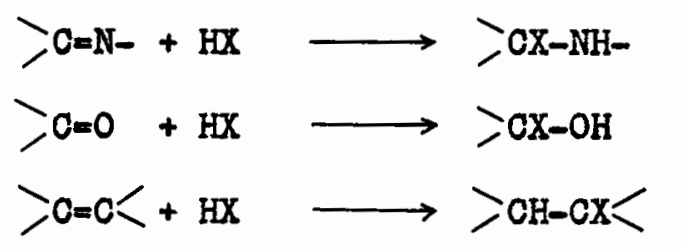

leading to substitution at position 6 , occurred in basic solutions, where $H$ was a proton, removed from kojic acid, and $X$ was the kojate anion in its "diketo" form (LXIV). Barchielli (86) studied the reaction between kojic acid (II) and Schiff bases (LXXI) in pyridine. Addition<smiles>[R]C=N[AsH3]</smiles>

LXXI<smiles>O=c1ccocc1</smiles>

II

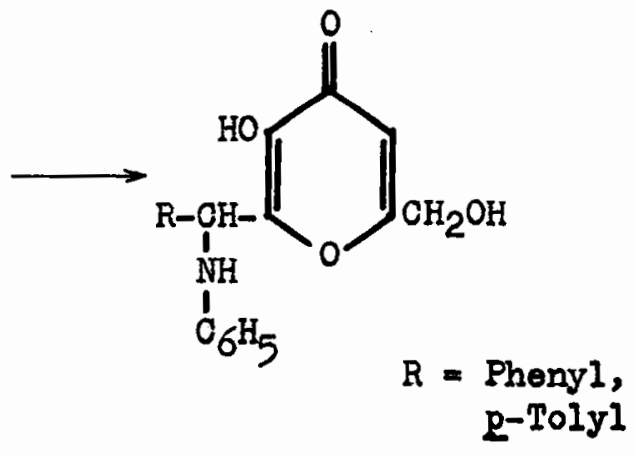

LXXII

occurred according to scheme A, giving products of structure LXXII. 
Woods found that kojic acid reacted with formaldehyde (87), acetaldehyde (88), glyoxal (89) and ethyl levulinate (90) according to scheme B, and with acrylonitrile (91) and crotonic acid (85) according to scheme C.

Ethyl levulinate was the only ketone which reacted with kojic acid in this manner, giving $4-\{6-[5$-hydroxy-2-(hydroxymethyl $)-\gamma$-pyronyl $]\}-$ - $\gamma$-valerolactone (LXXIV); the addition was presumably followed by lactonization on acidification of the reaction mixture. The primary product, $4-(6-[5$-hydroxy-2-(hydroxymethyl)- - -pyronyl $])$-4-hydroxyvaleric acid (LXXIII), was not isolated.

A more general reaction between kojic acid and aldehydes is a trimolecular condensation discovered by Barham and Reed (92). By a process of elimination, they arrived at the conclusion that position 6 of kojic acid was the most probable point of attack; two molecules of kojic acid reacted with one molecule of the aldehyde, with the elimination of one

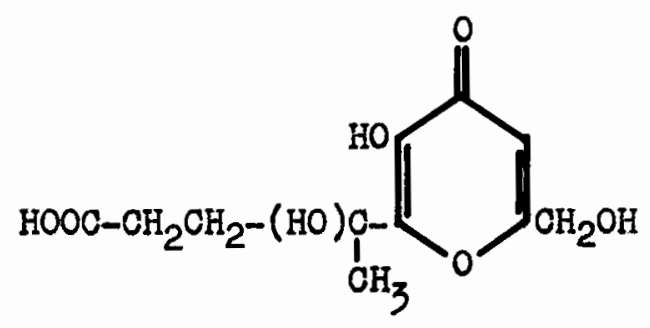

LXXIII

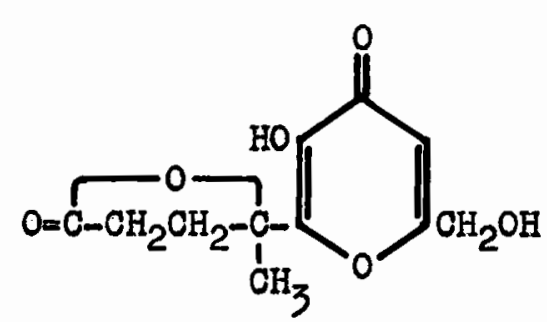

LXXIV<smiles></smiles>

LXXV

molecule of water, giving a product of structure LXXV. Such compounds 
were prepared from kojic acid and the following aldehydes: the normal alkanals from formaldehyde to heptanal, benzaldehyde, cinnamaldehyde, hydrocinnamaldehyde, furfural and acrololn. The compound derived from kojlc acid and benzaldehyde (LXXV, $R=$ Phenyl) was also obtained by treating LXXII ( $R=$ Phenyl) with hot aqueous sodium carbonate $(86)$.

In most of these nuclear substitution reactions, kojic acid did indeed react like a phenol. An exception was the reaction with acrylonitrile, as noted by Woods (91). Phenols usually form cyanoethyl ethers under similar conditions, but the reaction of acrylonitrile with ketones leads to substitution of the $\alpha$-hydrogen atom (93). This consideration points to a predominance of the "diketo" form of the kojate anion (LXIV) in these reactions. There are many good reasons to believe that, in the formation of all of these derivatives, substitution actually took place at position 6; it should, however, be pointed out that this assumption still lacks confirmation by synthesis, or by appropriate degradation studies. The nuclear mono-substitution products of kojic acid are listed in Table IV, their functional derivatives in Tables $V$ and VI.

The only instance of simultaneous substitution at positions 3 and 6 of the nucleus of kojic acid was the case of the Mannich basos (LXXVI) reported by Woods (95). Attempts to introduce two hydroxymethyl groups

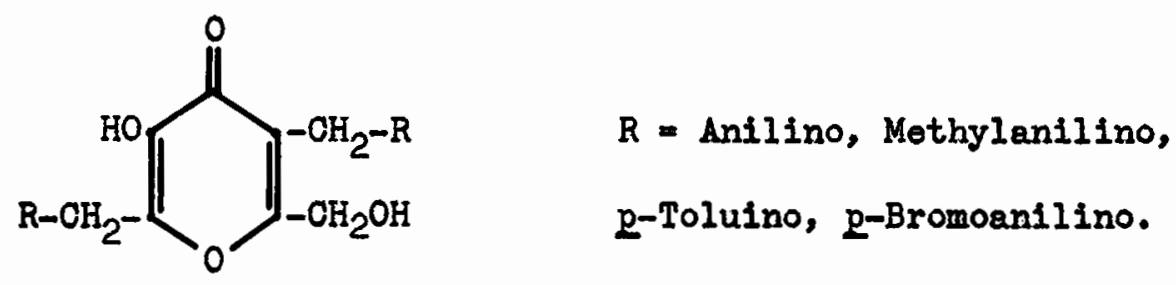

LXXVI

into the nucleus of kojic acid, under forcing conditions, gave mixtures 
TABLE IV

Nuclear Substitution Products of Kojle Acid<smiles>O=c1ccocc1O</smiles>

\begin{tabular}{|c|c|c|}
\hline $\mathbf{R}$ & $\begin{array}{l}\text { Melting } \\
\text { point }{ }^{\circ} \mathrm{C} \text {. }\end{array}$ & References \\
\hline 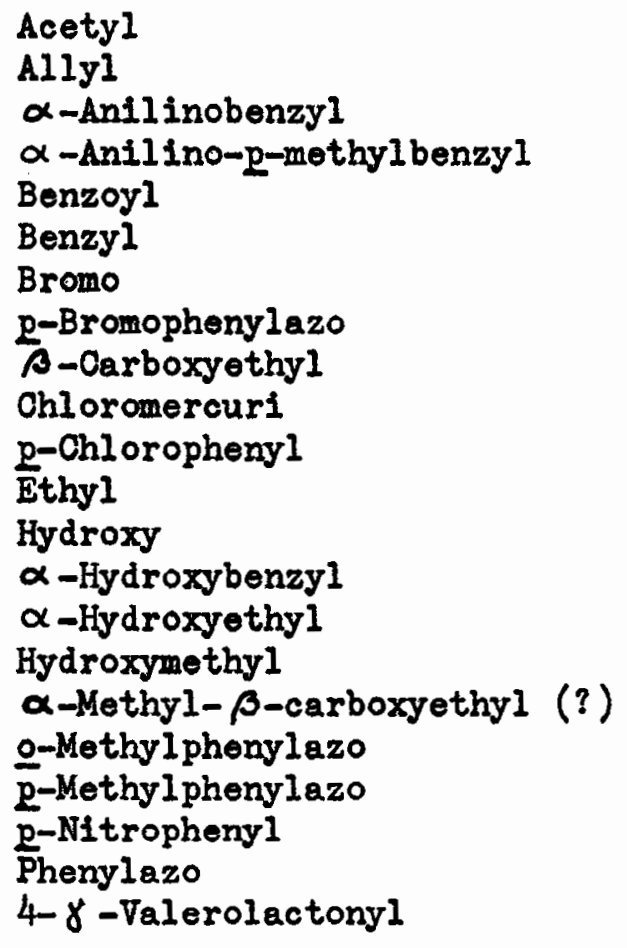 & $\begin{array}{l}156.5 \\
160-161 \\
176-177 \\
175 \text { dec. } \\
188 \\
78-80 \\
159-160 \\
145 \text { dec. } \\
155 \\
- \\
194 \\
147-149 \\
= \\
114-115 \\
155 \\
155-156 \\
154.5 \\
160-165 \text { dec. } \\
290 \text { dec. } \\
243 \\
146-147 \\
153.5\end{array}$ & $\begin{array}{l}84 \\
71 \\
86 \\
86 \\
83 \\
83 \\
4,5 \\
80 \\
85,91 \\
94 \\
80 \\
84 \\
4 \\
83 \\
88 \\
87 \\
85 \\
80 \\
80 \\
80 \\
4,79 \\
90\end{array}$ \\
\hline
\end{tabular}




\section{Mixed Derivetives of Kojic Acid (a)}<smiles></smiles>

\begin{tabular}{|c|c|c|c|c|}
\hline $\mathbf{R}$ & $R^{\prime}$ & $\mathrm{R}^{\prime \prime}$ & $\begin{array}{l}\text { Melting } \\
\text { point }{ }^{\circ} \mathrm{C} .\end{array}$ & $\begin{array}{l}\text { Refer- } \\
\text { ences }\end{array}$ \\
\hline 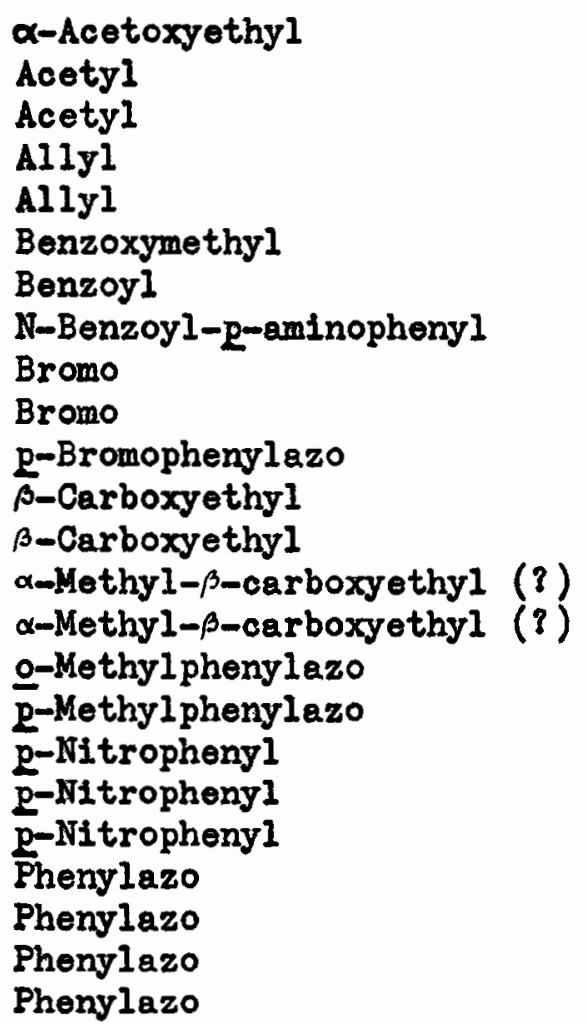 & $\begin{array}{l}\text { Acetyl } \\
\text { H } \\
\text { Acetyl } \\
\text { Acetyl } \\
\text { Allyl } \\
\text { Benzoyl } \\
\text { Acetyl } \\
\text { Benzoyl } \\
\text { Acetyl } \\
\text { Benzoyl } \\
\text { Methyl } \\
\text { Acetyl } \\
\text { p-Bromophenacyl } \\
\text { Acetyl } \\
\text { p-Bromophenacyl } \\
\text { Methyl } \\
\text { Methyl } \\
\text { Benzoyl } \\
\text { Methyl } \\
\text { Methyl } \\
\text { Methyl } \\
\text { Methyl } \\
\text { Methyl } \\
\text { Methyl }\end{array}$ & $\begin{array}{l}\text { Acetyl } \\
\text { Acetyl } \\
\text { Acetyl } \\
\text { Acetyl } \\
\text { H } \\
\text { Benzoyl } \\
\text { Acetyl } \\
\text { Benzoyl } \\
\text { Acetyl } \\
\text { Benzoyl } \\
\text { H } \\
\text { Acetyl } \\
\text { H } \\
\text { Acetyl } \\
\text { H } \\
\text { H } \\
\text { H } \\
\text { Benzoyl } \\
\text { H } \\
\text { Benzoyl } \\
\text { H } \\
\text { Acetyl } \\
\text { Benzoyl } \\
\text { Methyl }\end{array}$ & $\begin{array}{l}136.5 \\
119-120 \\
98-99 \\
112.5-113 \\
96-97 \\
134 \cdot 5-135.5 \\
182 \\
194 \\
94-95 \\
133-134 \\
200-202 \text { dec. } \\
85-87 \\
179-180.5 \\
133-135 \\
182.5 \\
176 \\
167-168 \\
145-146 \\
145 \\
180 \\
170 \\
133 \\
128 \\
124\end{array}$ & $\begin{array}{l}88 \\
84 \\
84 \\
71 \\
71 \\
87 \\
83 \\
80 \\
4 \\
4 \\
80 \\
91 \\
91 \\
85 \\
85 \\
80 \\
80 \\
80 \\
80 \\
80 \\
79 \\
79 \\
79 \\
79\end{array}$ \\
\hline
\end{tabular}


Mixed Derivatives of Kojic Ac1d (b)<smiles></smiles>

\begin{tabular}{|c|c|c|c|}
\hline R & $R^{\prime}$ & $\begin{array}{l}\text { Melting } \\
\text { point }{ }^{\circ} \mathrm{C} .\end{array}$ & $\begin{array}{l}\text { Refer- } \\
\text { ences }\end{array}$ \\
\hline $\begin{array}{l}\text { Acetyl } \\
\text { Benzoyl } \\
\text { Bromo } \\
\text { Bromo } \\
\text { o-Carboxyethyl } \\
\text { o-Chloroethyl } \\
\text { Hydroxymethyl } \\
\text { Phenylazo } \\
4-\gamma \text {-Valerolactonyl }\end{array}$ & $\begin{array}{l}\text { Chloro } \\
\text { Chloro } \\
\text { H } \\
\text { Chloro } \\
\text { Chloro } \\
\text { Chloro } \\
\text { Chloro } \\
\text { H } \\
\text { Chloro }\end{array}$ & $\begin{array}{l}163-164 \\
185-187 \\
171-173 \\
163 \\
164.5 \\
166.5 \\
165 \\
- \\
167-169\end{array}$ & $\begin{array}{l}84 \\
83 \\
8 \\
81 \\
85 \\
89 \\
87 \\
8 \\
90\end{array}$ \\
\hline
\end{tabular}


of inconclusive composition (87).

The Nuclear "Double Bonds":- Substances that usually condense with carbonyl groups, e.g. hydroxylamine or phenylhydrazine, rarely react with $\gamma$-pyrones (96), and the carbonyl group in position 4 of kojic acid has so far yielded no condensation products of this kind. Neither has the addition of carbonyl reagents to the carbon-to-carbon double bonds of the nucleus of kojic acid been reported, although such additions are not uncomon with $\alpha, \beta$-unsaturated ketones of the aliphatic and alicyclic series. There exists, indeed, very little evidence as to the ability of these double bonds to add reagents. The addition of two atoms of iodine at each of the two carbon-to-carbon double bonds was postulated by Airoldi and Bionda (51) from the results of titrations with iodine, and the addition of two atoms of bromine per double bond was assumed by wijkman (6). In neither case were the addition products isolated. Their existence would seem to be at least questionable. As mentioned earlier, Yabuta (4) observed the mono-substitution of kojic acid by bromine water, and Musante (68) reported the degradation of kojic acid by the same reagent, but no bromination. The addition of hydrogen to the nuclear double bonds of kojic acid will be considered in a separate section.

The Hetero-Atom of the Nucleus:- The cleavage of many 8 -pyrones (LXXVII) at the ring oxygen atom has been observed in mildly alkaline solutions $(66,96)$. The primary products of this reaction were labile bis-hydroxymethylene compounds (LXXVIII), which became stabilized in some cases by a tautomeric shift to their ketonic forms (LXXIX), or were reconverted to the cyclic form (LXXVII) by acidification. No 
<smiles></smiles>

LXXVII<smiles>[R]C(O)=CC(=O)C([R])O</smiles>

LXXVIII<smiles>[R]C(=O)CC(=O)O[Po]</smiles>

LXXIX

studies were carried out with kojic acid in this direction. Quilico and Musante $(80)$ observed the cleavage of the $\gamma$-pyrone ring of various 6-arylazokojic acids (LXV) by $20 \%$ aqueous sodiun hydroxide, by aniline and by phenylhydrazine. They postulated open chain structures for the products, but lacked sufficient chemical evidence to confirm them. The cleavage of kojic acid by hot alkali to fragments containing one to three carbon atoms could not be definitely shown to commence at the ring oxygen atom, but what was said earlier about $\gamma$-pyrones in general renders this conclusion probable.

The initiation of the basic clearage at the ring oxygen atom is further indicated by the easy formation of 4(1트)-pyridones when kojic acid and several of its derivatives are condensed with primary amines or amonia (4, $7,8,10,75,79)$. The reaction with amonia is usually carried out under pressure (10), but with amines it takes place in boiling (75), or even in cold (10), solutions. No intermediates have been isolated from these reactions, but it seems likely that they would be analogous to LXXVIII.

Oxidation:- The oxidation of the hydroxymethyl sidechain of some kojic acid derivatives to a carboxyl group, and the failure to oxidize kojic acid in the same way, was discussed earlier. Heyns and Vogelsang (16) recently attempted to achieve this oxidation catalytically. A 
current of air was passed through solutions of kojic acid (II) and its 5-methyl ether $(X)$ at $60^{\circ} \mathrm{C}$. , for several hours; the solutions were kept close to $\mathrm{pH} 5$, and a special platinum - activated carbon catalyst wos used. Only traces of comenic acid (IV), demonstrated by paper chromatography, were obtained from II; the yields of oomenic acid methyl ether (XI) from $X$ were close to $40 \%$.

Obata and Yamanishi (97) employed several mild oxidizing agents, without success, in their attempts to oxidize the hydroxymethyl group of kojic acid to a formyl group.

The oxidative degradation of kojic acid by bromine water was mentioned earlier. Yabuta reported the degradation of kojic acid (3) and its 5-benzoate (8) by alkaline potassium permanganate. Kojic acid was also oxidized by Fehling's solution and by molst silver oxide, according to qualitative tests (4), and these reactions were quite likely accompanied by degradation. Oxidative breakdown by aqueous alkaline hypoiodite formed the basis of a volumetric method for the quantitative estimation of kojic acid. Birkinshaw and Raiatrick (50) studied this reaction in detail, and isolated the following cleavage products: formic acid, oxallc acid and glycolic acid. They postulated the following scheme for the breakdown, which resembled closely the degradation of the ethers of kojic acid by aqueous alkali.

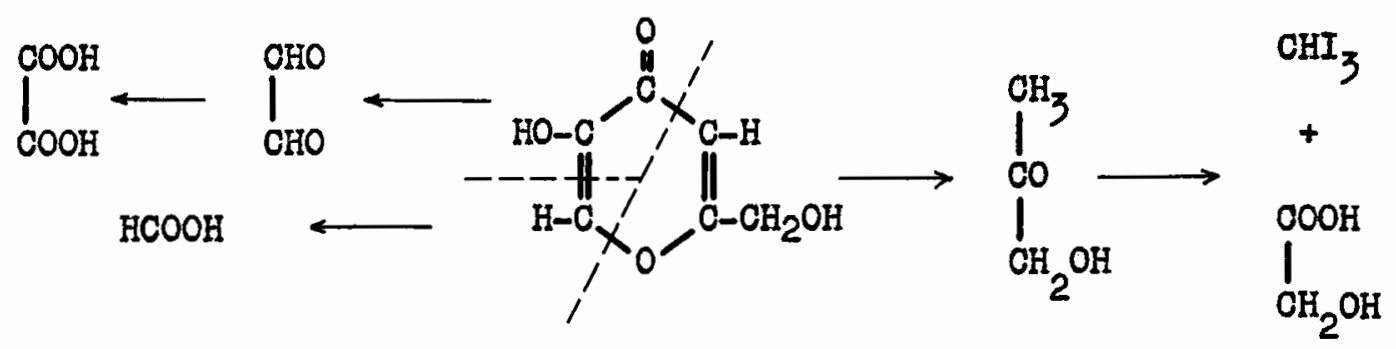


Reduction:- The catalytic hydrogenation of kofic acid was studied repeatedly. Traetta Mosca (5) used palladium in alcohol and observed the uptake of four atoms of hydrogen; Wifkenen (6) employed platimum as well as pelladium, and found that six atoms of hydrogen were absorbed. Both workers reported a eirupy product, of undetermined composition. Armit and Nolan (10) hydrogenated kojic acid and its dimethyl ether over colloidal palladium, and diacetylkojic acid (XIII) with colloidal platinm, at a pressure of 2 atmospheres in each case. The first two compounds each absorbed six atoms of hydrogen, the last one ten atoms, and once more all three products were simps and oils of inconclusive composition. The oily hydrogenation product of XIIII reacted with phenylhydrazine and formed a crystalline osazone, with the probable structure of LXXXI.<smiles>O=C1C[C@H](CO)OCC1O</smiles>

LXXX<smiles>O=[NH+]NC1COC(CO)CO1</smiles>

LXXXI, R $=$ AcetyI LXXXII, $R=H$

Maurer (59) alone identified a crystalline derivative of tetrahydrokojic acid ( $L X X X)$, which was a sirup prepared by permitting kofic acid to absorb four atoms of hydrogen at 1 atmosphere pressure, and in the presence of colloidal palladium. Although this sirup was not analysed, it yielded a crystalline osezone (LXXXII), the structure of which was confirmed by synthesis. Maurer's results rendered very likely the correctness of the structure assigned by Armit and Nolan to LXXXI. The non-catalytic reduction of kojlc acid was never attempted, but 
some conclusions concerning the probable results may be drawn from the following observations. Woods reduced 6-benzoylkojic acid (LXXXIII) by the Clemensen method to G-benzylkojic acid (LXXXIV)(83), and likewise 6-acetylkojic acid (LXXXV) to 6-ethylkojic acid (LXXXVI) (84); the reduction of LXXXIII by the Meerwein-Ponndorff-Verley method yielded $6-(\alpha$-hydroxybenzyl)-kojic acid (LXXXVII)(83). Quilico and Musante (80)

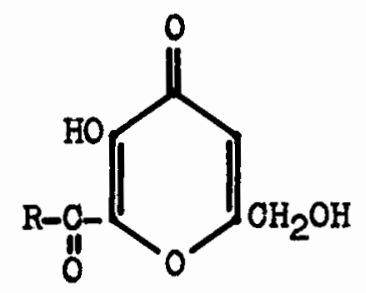

LXXXIII, $R=$ Phenyl LXXXV, $R=$ Methy1<smiles>[R6]Cc1cc(=O)cc(O)o1</smiles>

LXXXIV, $R=$ Phenyl LXXXVI, $R=$ MethyI<smiles></smiles>

LXXXVII

reduced 6-p-nitrophenylkojic acid ( $\mathrm{LXVII}, R=\mathrm{NO}_{2}$ ) with stannous chloride and concentrated hydrochloric acid to 6-p-aminophenylkojic acid (LXVII, $R=\mathrm{NH}_{2}$ ). In none of these reactions was the nucleus of kojic acid affocted.

Miscellaneous Derivatives:- A few derivatives of kofic acid, which could not readily be included in the earlier sections, will be mentioned heres a hexathiocyanate, obtained by Woods (98) from a reaction between kojic acid, potassium thiocyanate and bromine in acotic acid; the condensation product from one molecule of kojic acid and two molecules of o-phenylenediamine, reported by Maurer and co-workers (99); the sulfide, propared by Yabuta (4) from the lead double salt of 6-bromokojic acid and hydrogen sulfide; a crystalline complex containing both sodium and bromine, formed from two molecules of sodium kojate and two molecules of phenacyl bromide in anhydrous methanol, discovered by Hurd and Sims (71); 
finally, some derivatives of kojic acid with unconfirmed structures, reported by Woods $(83,84,89,90,100)$. Kojic acid was ouggested as a developer for inorganic paper chromatography by Pollard and co-workers (101).

\section{Physical Properties}

Kojic acid crystallizes in the form of prismatic white needles (3, 5). Data on its crystal stmucture, derived from X-ray investigations, were provided by Fox (102), and by McKinstry and co-workers (103).

Kojic acid is readily soluble in water, ethanol and acetone; oparingly soluble in ether, ethyl acetate, chloroform and pyridine; and scarcely at all in most other liquids $(4,7)$. It has been purified by crystallization from acetone (7), ethanol-ether (20) and methanolethyl acetate (26); also by sublimation at reduced pressures and at 150$200^{\circ}$ c. $(6,26,54)$. The melting point was given (in ${ }^{\circ} \mathrm{C}$. ) as : 151-152 $(20,42), 152(3,21,54), 152.6(37), 152-153(43), 153-154(29,31)$ and $154(5,6)$. The molecular weight was determined by the cryoscopic method (4, 6, 7, 31) and the ralues obtained agreed well with the calculated value of 142 .

The ultra-violet absorption spectra of kojic acid and its 5,7-diacetate were determined by Stacey and Turton (63), the characteriatic absorption maximum of kojic acid being located at $\lambda 3150 \AA$, and that of the diacetate at $\lambda 2550 \AA$. Kuhn (104) messured the absorption of kojic ac1d in the infre-red.

Paper chromatography data were provided by Heyns and Vogelsang (16). 
Kojic acid: $R_{f}, 0.80$; developer, butanol (saturated with water) glacial acetic acid - water, 18:2:5; developing time, 22 hours; spraying reagent, $0.1 \%$ ferric chloride; paper, Schleicher \& Schüll, No. $2043 \mathrm{~b}$.

\section{Biological Properties}

Antibiotic Activity:- Yabuta (3) noted that bacterial growth generally stopped in the presence of more than $0.5 \%$ of kojic acid, but interest in this antibacterial activity was only renewed quite incidentally when the discovery of penicillin led to an intensified search for new antibiotics among mold metabolites. The promisingly high activity of some mold culture media against rarious bacteria was found to be due wholly to the relatively high concentration of kojic acid produced by these molds (22, 23, 41, 105), but the inhibitory power of pure kojic acid $(21,41,106,107,108)$ and of some of its derivatives $(73,107)$ proved to be disappointingly low. The potency of kojic acid was unaffected (41) by the number of bacteria present, or by incubation in $50 \%$ serum at $37^{\circ}$ C. Foster and Karow (109), who also noted a slight inhibition of pure cultures of various bacteria by kojic acid, made the unexpected observation that the gram-negative organisms were more sensitive to sodium kojate than were the gram-positive. With most other antibiotice the reverse was true. Kavanagh (110) compared the activity of kojic acid with several of the well-known potent antibiotics, and kojic acid showed by far the lowest activity in the whole group against seven out of nine species of bacteria. Lee and co-workers (105) found that kojic acid was active againgt human tubercle bacilli in vitro under a variety of conditions, complete inhibition of a surface growth of the bacilli being 
caused by $45 \mathrm{mg}$. per $100 \mathrm{ml}$. of liquid medium. However, many other active substances produced an equal effect at much lower concentrations. The effect of kojic acid at inhibitory levels was bacteriostatic, not bactericidal.

Kofic acid is not yet known to be active against viruses. It was tested, with negative results, against one strain of poliomyelitis virus and one strain of St. Louis encephalitis virus in mice (111), and also against sixty races of bacteriophage (112).

Klein and Olsen (113) studied the action of kojic acid on the enzymatic oxidation of amino acids by the liver and kidney of rats. Low concentrations of kojic acid inhibited in vitro the oxidation of a mmber of D-amino acids, I-phenylalanine and a few related compounds. Kojic acid wes found to compete with D-amino acid oxidase for the substrate. Although McGowan and co-workers (114) observed only a weak activity of kojic acid against three species of fungi, O'Kane and Morey (115) recommended the use of its complexes with bivalent heary metals (LIX) against several fungus diseases of plants. They found the coppor, mercury, cadmium, lead and tin complexes to be superior to the well-known Bordeaux mixture (cupric sulfate - calcium oxide - vater) in the treatment of early blight (Alternaria solani). These complexes were easily suspended in liquids, their sprays adhered firmly to the follage and did not injure the plant. Since the metal radicals were bound to kojic acid In a non-ionizable forn, these complexes were also much less toxic to human beings and safer to handle than Bordeaux mixture. Thus, the activity of kojic acid against certain fungi may eventually find some practical application. 
Mayer and co-workers (116) found kojic acid to be a moderately effective activator for nicotine insecticides. When tested against the melon worm (Diaphania hyalinata L.) and the southern armyworm (Prodenia eridania (ram.), kojic acid alone was not toxic; but the toxicity of a $5 \%$ nicotine sulfate - pyrophyllite dust was increased $35 \%$, and that of a $5 \%$ nicotine bentonite - bentonite spray 50\%, by the addition of $5 \%$ of kojic acid. These workers found kojic acid harmless to plants, but others (117) found it slightly toxic.

Toxicity:- Indication of the toxicity of kojic acid to mamals was given by Friedemann (118), who observed a definite response in dogs after intravenous injection of $0.15 \mathrm{~g}$. of sodium kojate per $1 \mathrm{~g}$. of body weight, and found the lethal dose to be about $1 \mathrm{~g}$. per $1 \mathrm{~kg}$. of body weight. Practically the same symptoms were shown by rabbits and rats. Toxicity to mice of the same order was reported by Morton and co-workers (2I) and by Jennings and Williams (4I). The latter workers found further that humen leucocytes were killed within three hours by a $1 \%$ solution of sodium kojate at $\mathrm{pH}$ 6.8. Twelve day old chick embryos were also susceptible to kojic acid, the observed $L D_{100}$ being $12 \mathrm{mg}$. per $100 \mathrm{~g}$. of egg weight (105). A moderate cardiotoxic (119) and cardiotonic (120) activity of kojic acid was also observed. In none of these instances was the toxicity very great, but it was enough to discourage chemotherapeutic investigations and to prevent the medicinal use of kojic acid. 
DISCUSSION OF RESULTS

The above survey of the chemical properties of kojic acid revealed two areas in which information about its reactivity was particularly scanty. Practically nothing was known about the possibility of ring cleavage at position 1, leading to the formation of acyclic polyfunctional hexane derivatives. The other neglected aspect was the ability of kojic acid to condense with the so-called carbonyl reagents, primarily with the various substituted hydrazines. Analogous reactions of other $\gamma$-pyrones have been described, and brief summaries of those studies are given at the beginnings of the appropriate sections of this discussion.* That work provided some guidence for the following researches on kojic acid.

\section{Attempted Ring Cleavage of Kojic Acid at Position 1}

$$
\text { Analogous Reactions of Other } \gamma \text {-Pyrones }
$$

It was mentioned earlier that alkaline reagents frequently cleaved the ring of $\gamma$-pyrones, forming unstable open chain dienolic compounds. A few characteristic examples will now be considered briefly. The only 
product actually isolated as the dienol appears to have been Haitinger and Lieben's (121) "xanthochelidonic acid" (LXXXVIII), which was obtained in solution a a tri-basic yellow salt when chelidonic acid (LXXXIX) was<smiles>CCOC(=O)C=C(O)C(=O)O</smiles>

LXXXVIII, $R=H$ $X C I, R=$ Ethyl

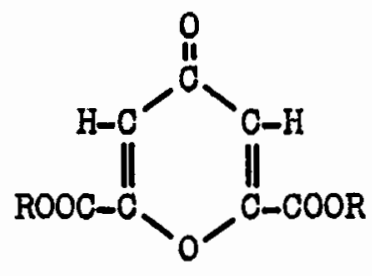

LXXXIX, R $=\mathrm{H}$ XCII, $R=$ Ethyl<smiles>CCOC(=O)CC(=O)OC(=O)OC(=O)OCc1ccccc1</smiles>

XC, $R=$ Ethy 1

treated with three equivalents of cold aqueous potassium hydroxide. When the solution of the salt of LXXXVIII was acidified and extracted with ether, a yellow substance was recovered, presumably the free acid (IXXXVIII). This substance was very labile, and had a definite tendency to revert to chelidonic acid (LXXXIX). If the acidified solution was left standing at room temperature, the yellow color gradually disappeared, and the reversel to LXXXIX went to completion. Diethyl acetonedioxalate (XC), the fairly stable ester of the hypothetical triketo form of LXXXVIII, was synthesized by Claisen (122) from acetone and diethyl oxalate; the diester could be cyclized readily to ethyl chelidonate (XCII), with dehydrating agents. Crystalline mono- and dienolic forms of XC were reported by Willstätter and Pumerer (123); the exact structure for these isomers was not determined, but the yellow dienol might well have been identical with the unknown "ethyl xanthochelidonate" (XCI). If the $\gamma$-pyrone ring was part of a condensed ring system, then one or both of the hydroxyl groups created by cleavage at position I would be phenolic, rather than enolic, and the cleavage product would 
consequently be stable. Such was the case with khellinone (XCIV), obtained by Schönberg and Sina (124) from khellin (XCIII) by treatment with hot alcoholic potassium hydroxide. The other enolic hydroxyl group, presumably formed initially, appeared as the more stable methyl ketone.<smiles></smiles>

XCIII<smiles>COc1ccc(C(=O)CC(C)=O)c2ccoc12</smiles>

XCIV

With most $\gamma$-pyrones, however, the cleaved forms were not stable enough to exist as such, and their transitory existence could only be proved by converting them, without prior isolation, to stable enol ethers or enol esters. Willstätter and Pumerer (125) demonstrated the cleavage of $\gamma$-pyrone (XCV) itself in aqueous alkali by preparing from it the

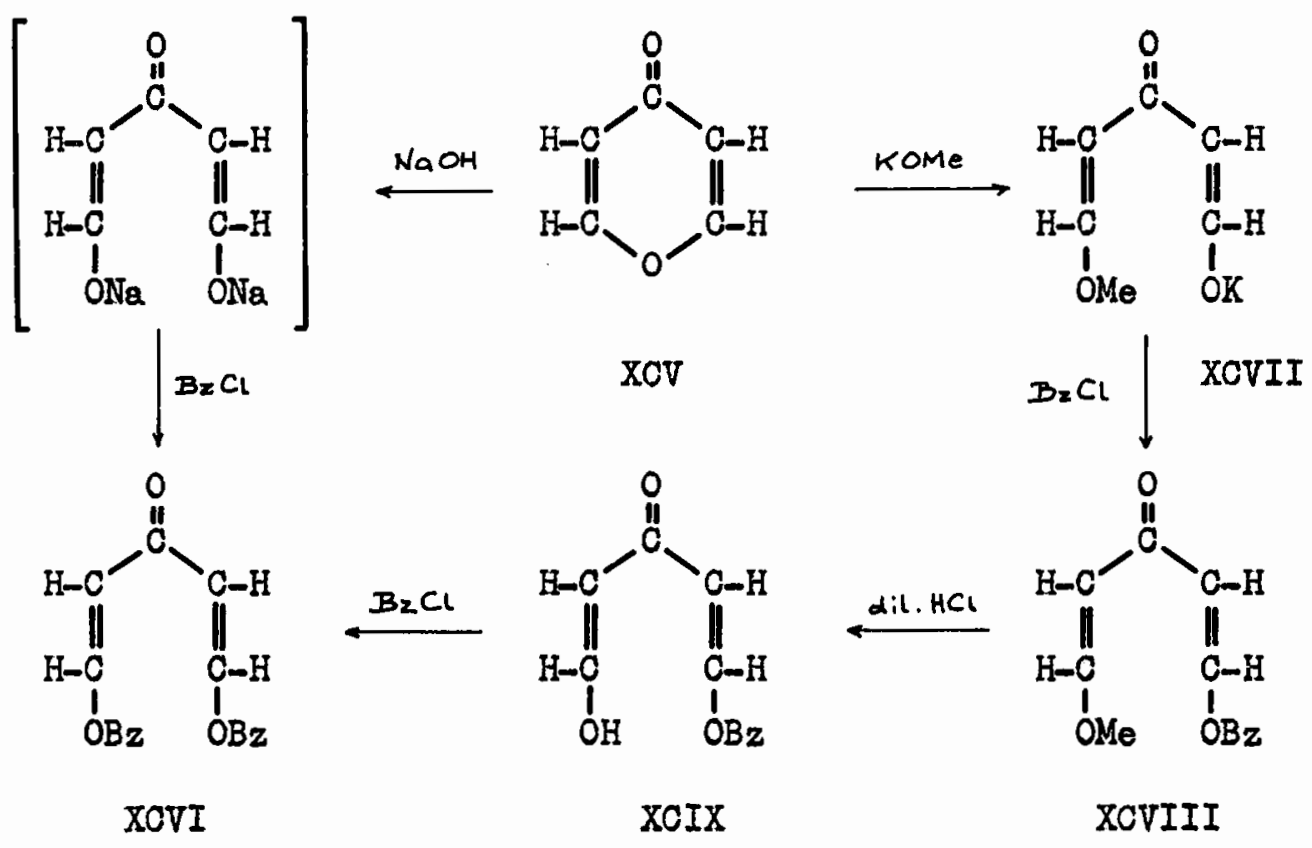


following derivatives of bis-(hydroxymethylene)-acetone: the dibenzoate (XCVI), the potassium salt of the monomethyl ether (XCVII), the benzoate of the monomethyl ether (XCVIII), and the monobenzoate (XCIX).

\section{Resistance of Kojic Acid to Aqueous Alkali}

If kofic acid (C) were to undergo such a ring cleavage, the initial dienolic product (CI) would probably come to an equilibrium with its keto-enolic (CII, CIII) and ketonic (CIV) tautomers. These might, or might not, be stable enough for isolation; they might, however, be converted to stable enolic derivatives.

When exposed to alkali of more than a certain concentration, usually two normal, the cleavage of $\gamma$-pyrones did not stop at these open chain forms, but led to the fragnentation of the molecule. In fact, the only known cleaving effect of alkalies on kojic acid was a degradation to fragments containing one to three carbon atoms $(4,7,55)$. Before the isolation of derivatives like CI to CIV could be attempted, the resistance of kojic acid to cold aqueous alkali of varying concentrations had to be explored.

Solutions of kojic acid were accordingly made up in varying volumes of approximately normal aqueous sodium hydroxide (a).* These yellow solutions were allowed to stand at room temperature for periods of from one to thirty-two hours, and were then acidified with $20 \%$ sulfuric acid. The colorless, acidic liquids were then chilled and seeded to recover

* The small letters in parentheses refer to the appropriate procedures in the Experimental section. 
<smiles>C=CCC(=O)CC(=O)C(O)=C(O)CO</smiles>

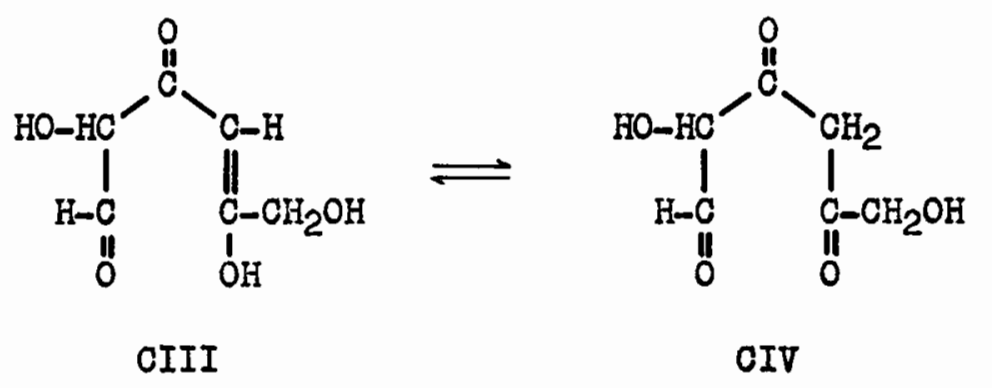

the unchanged kojic acid as a crystelline deposit. In some cases a second crop of kojic acid was obtained by concentration of the filtrates; in others the formic acid formed by the degradation of kojic acid was recovered by distillation and estimated by titration with standard alkali. Each mole of formic acid produced corresponded to one mole of kojic acid degtroyed. The resulta of these tests are collected in Table VII. For all practical purposes, kojic acid could be considered stable in normal aqueous sodium hydroxide at room temperature, since at least $74 \%$ was recovered, and ten milliliters of the final nomel sodium sulfate solution was known to dissolve about 0.15 gram (15\%). Even a fivefold excess of alkall, acting for thirty-two hours, caused the degradation of only slightly over $3 \%$ of the kojic acid. If the yellow color of the alkaline solution indicated the presence of an enolic cleavage product, the disappearance of the color and the almost quantitative recovery of kojic acid proved that it had completely reverted to the original cyclic form. 
TABLE VII

Treatment of Kojic Acid with Normal Aqueous

Sodium Hydroxide at Room Temperature

\begin{tabular}{|c|c|c|c|c|c|c|c|}
\hline $\begin{array}{l}\text { Moles } \mathrm{NaOH} \\
\text { per I Mole of } \\
\text { Kojic Acid }\end{array}$ & $\begin{array}{c}\text { Time of } \\
\text { Treatment } \\
\text { hr. }\end{array}$ & $\begin{array}{c}\text { Volume of } \\
\text { Solution } \\
\text { ml. }\end{array}$ & lst & $\begin{array}{l}\text { Kojic Acid Re } \\
\text { Crop (b) 2nd } \\
\text { g. }\end{array}$ & $\begin{array}{l}\text { ecovered } \\
\text { Crop (c) } \\
\text { g. }\end{array}$ & $\begin{array}{l}\text { a) } \\
\text { Total } \\
\text { g. }\end{array}$ & $\begin{array}{l}\text { Formic Acid } \\
\text { in Distillate } \\
\text { mole } \%\end{array}$ \\
\hline 2 & 1.2 & 11.3 & & 0.76 & 0.13 & 0.89 & ... \\
\hline 2 & 3.0 & 11.3 & & 0.73 & 0.15 & 0.88 & ... \\
\hline 2 & 13.5 & 11.3 & & 0.77 & ... & ... & 1.0 \\
\hline 2 & 32.0 & 11.3 & & 0.74 & ... & ... & 2.5 \\
\hline 3 & 1.0 & 16.9 & & 0.66 & 0.19 & 0.85 & ... \\
\hline 3 & 32.0 & 16.9 & & 0.74 & ... & ... & 3.2 \\
\hline 5 & 1.0 & 28.2 & & 0.56 & 0.25 & 0.81 & ... \\
\hline
\end{tabular}

(a) One gram of kojic acid was used in each test.

(b) Obtained by chilling the acidified solutions to $-3^{\circ}$.

(c) Obtained by concentrating the filtrate to two fifths of its original volume and chilling to $-3^{\circ}$. 
In order to verify the existence of the hypothetical open chain cleavage product, the formation of derivatives had to be attempted in the alkaline solution.

It was found that the sodium salt of kojic acid could be obtained readily by the complete evaporation of an aqueous solution of kojic acid which had been neutralized with sodium hydroxide (b). When recrystallized from water, sodium kojate formed white prismatic needles and contained four molecules of water of crystallization, which were lost readily at $110^{\circ}$. The hydrated salt was kept at room temperature for several months without signs of deterioration. That this was not a sodium, or disodium, salt of an enolic open chain form was indicated by the sodium content, and by the formation of a normal derivative of kojic acid from anhydrous sodium kojate in a dry, non-polar solvent. This reaction will be discussed fully in a subsequent section.

\section{Methylation Studies}

Methylation with dimethyl sulfate in $10 \%$ aqueous potassium hydroxide was tried as a method of stabilizing the supposed enolic open chain derivatives of kojic acid. This standard reaction had been applied to kojic acid before. Campbell and his co-workers (75) reported that the use of one equivelent of dimethyl sulfate and slightly more than one equivalent of potassium hydroxide at $20^{\circ}$ gave a $72 \%$ yield of crude 2 -(hydroxymethyl)5-methoxy- $\gamma$-pyrone $(\mathrm{CV})$, one of the normal monomethyl ethers of kojic acid. While no details were given in Chemical Abstracts, it is likely that Yabuta (4) used two equivalents of each of the two reagents to 
<smiles>COc1coc(O)cc1=O</smiles>

CV<smiles></smiles>

CVI<smiles></smiles>

CVII

prepare the dimethyl ether of kojic acid (CVI). In the present work it was hoped to obtain a tri- or tetra-0-methyl open chain derivative of kojic acid by applying between three and four equivalents of dimethyl sulfate, and somewhat greater excess of alkali (c). The reaction was allowed to proceed for three hours at $25-30^{\circ}$. The enol ethers were expected to be neutral compounds, capable of extraction from the alkaline liquor by benzene. Any of the neutral cyclic ethers CV and CVI formed in the methylation would be recovered at the same time. However, continuous extraction of the liquor with benzene yielded only traces of oily impurities. Acidification and re-extraction of the liquor gave a $45 \%$ by weight yield of a crude solid product, which was found to contain a white crystalline solid of m.p. $75-76^{\circ}$, and a pale yellow crystalline solid melting at $270-271^{\circ}$ with decomposition. The former substance gave the characteristic color reaction of enols and phenols with ferric chloride, and was found to have the composition of a normal monomethyl ether of kojic acid. Consequently, this substance had to be 5-hydroxy2-(methoxymethyl)- $\gamma$-pyrone (CVII), the second, hitherto unknown, monomethyl ether. The identity of CVII was definitely proved by methylation with diazomethane (e), to give CVI. The pale yellow, high-melting product left a small amount of ash on ignition, and the ash contained aluminum. Acidification of an aqueous solution of the solid, followed 
by the extraction of the organic solute, yielded CVII (d). From this result, and from analytical data, the solid was recognized as the anhydrous aluminum salt of CVII, with a composition corresponding to Al $\left[\mathrm{C}_{6} \mathrm{H}_{4} \mathrm{O}_{3}\left(\mathrm{CH}_{3}\right) 3_{3}\right.$. This salt was sparingly soluble in water and in organic liquids, gave a wine color with aqueous ferric chloride, and was probably best represented by an aluminum phenoxide type of structure (CVIII).<smiles></smiles>

The source of the aluminum was traced to an alumina chromatographic column which had been used in the purification of the crude products of the methylation (c). The total yield of CVII, including the portion recovered in the form of the aluminum salt, amounted to $15 \%$; the remainder of the crude product from the methylation defied further attempts at purification.

The failure to isolate open chain enol ethers could not be considered as a proof of the non-existence of open chain cleavage products, but their presence in alkaline solutions of kojic acid was rendered somewhat less likely. The methylation of the primary alcoholic hydroxyl group of kojic acid in preference to the phenolic hydroxyl group could be considered as a highly unusual result. As a rule, phenolic compounds react very readily with dimethyl sulfate and alkali; indeed, more readily than alcohols. It was conceivable that the "diketo" form of the kojate 
anion (LXIV) predominated at higher alkali concentrations, and permitted only the methylation of the hydroxyl group of the side chain. Another possible course of the reaction might have been initiated by the formation of the dimethyl ether (CVI), followed by alkaline hydrolyais of the methoxyl group at position 5. The methyl ethers of some highly acidic phenolic compounds are known to be unusually labile; for instance, 6-carbomethoxy-2,4-dimethoxy-5-cyclohexene-1,3-dione(CIX) was completely demethylated by $5 \%$ aqueous sodium hydroxide to give a erystalline compound with the composition of $2,3,4,5$-tetrahydroxybenzoic acid (CX)(126).<smiles>COC(=O)C1C(=O)C(C)C(=O)C1C</smiles>

CIX

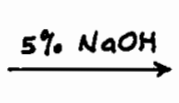<smiles>O=C(O)C1CCCCC1C(=O)O</smiles>

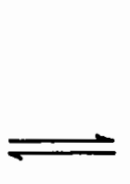

CX

Nevertheless, this second alternative seemed quite improbable because the degradation of $\mathrm{CV}(7)$ and of $\mathrm{CVI}(4,55)$ by boiling alkalies always produced fragments which still contained the methoxyl groups. In other words, the fragmentation of the ring would no doubt precede the cleavage of an ether linkage at position 5.

\section{Benzoylation Studies}

The reaction between benzoyl chloride and kojic acid was described in the literature; Yabuta (4) prepared dibenzoylkojic acid, m.p. 135-136 , from the two compounds in cold aqueous sodium hydroxide. It was decided, 
nevertheless, to study this reaction more closely with the hope of isolating onolic products of the alkaline cleavage of kojic acid by the formation of their benzoates.

In the first of the mothods tried $(f)$, kojic acid was dissolved in a tenfold excess of normal aqueous sodium hydroxide, and the solution was treated forty-eight hours later with five equivalents of benzoyl chloride. The medium was quite highly alkaline initially, and remained alkaline throughout the reaction. In the second method $(\mathrm{g})$, the initial excess of sodium hydroxide over kojic acid was fivefold and the reaction wes interrupted after the addition of four equivelents of benzoyl chlorIde to remove the insoluble products. Thereafter, the benzoylation was resumed by adding four more equivalents of sodium hydroxide, followed by four equivalents of benzoyl chloride. The liquor was acidic to litmus at the end of the benzoylation, and the purpose of the procedure was to keep the alkalinity low throughout the reaction, to protect any alkalisensitive enolic esters that might be formed.

The products of these benzoylations were the following: white crystals melting at $134-135^{\circ}$, and matted white needles melting at $120-121^{\circ}$ by the first method (total yield, 22\%); white needles melting at 142$143^{\circ}$, and again the substance of m.p. 120-121 $1^{\circ}$ by the second method (total yield, 53\%). The first substance (m.p. 134-135 ) was identified as dibenzoylkojic acid by a mixed melting point determination with an authentic sample, but the other two compounds had melting points corresponding to none of the known benzoyl derivatives of kojic acid. The substance melting at $142-143^{\circ}$ was found to have the composition of a monobenzoate of kojic acid; of the two possible monobenzoates, only 
one was recorded in the literature, namely 5-benzoxy-2-(hydroxymethyl)$\gamma$-pyrone (CXI), m.p. $135-136^{\circ}$, prepared by Yabuta $(4,72)$ using two different methods. Despite the discrepancy in melting points, these two compounds were almost cortain to be one and the same derivative, because the new substance gave, like CXI, no color reaction with ferric chloride, indicating the absence of a free phenolic hydroxyl group. To make a final decision possible, Yabuta's (72) somewhat laborious, but unequivocal, synthesis of OXI was repeated: 5-benzoxy-2-(triphenylmethoxymethyl)- $\gamma-$ pyrone (OXII) was prepared from kojic acid (II) in two steps ( $j)$, and detritylated with boiling $80 \%$ acetic acid ( $k$ ) to give a product melting at $142-143^{\circ}$, seven degrees above the value given by Yabuta (72). This

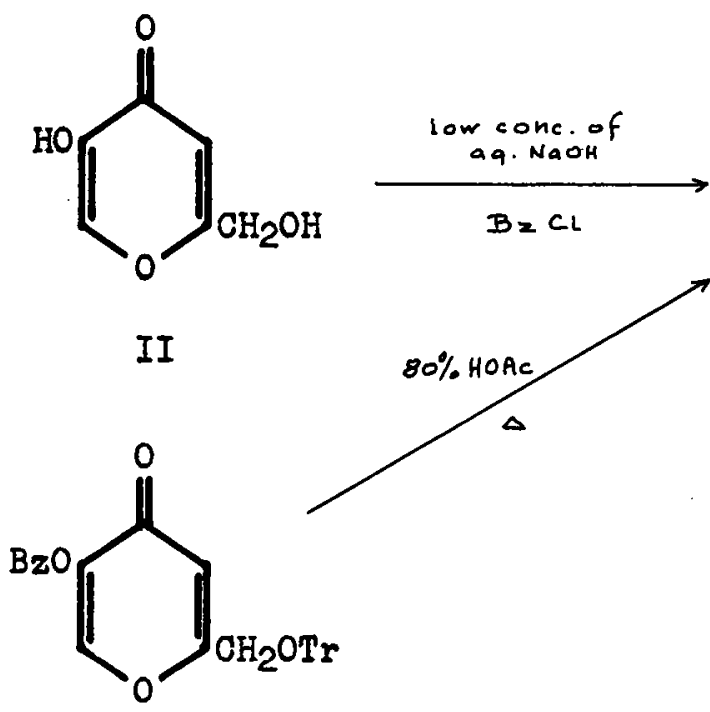

CXII<smiles>O=C(O)c1coccc1=O</smiles>

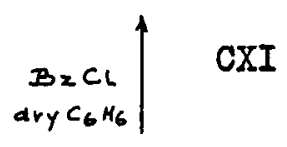<smiles>C[N+](C)(C)C1=COC(CO)CC1=O</smiles>

CXIII

melting point was not depressed by admixture with the product of m.p. $142-143^{\circ}$ from the benzoylation of kojic acid (II) in aqueous alkali ( $g$ ), and the identity of the latter derivative with CXI was thereby confirmed. Yabuta's (4) other method for the synthesis of CXI, consisting of boiling 
kojic acid (II) with benzoyl chloride in other, was found to be impractical, owing to the poor solubility of II in ether. Very pure CXI, melting at $143.5-144.5^{\circ}$, was prepared in close to $80 \%$ yield by shaking anhydrous sodium kojate (CXIII) and benzoyl chloride in dry benzene at room temperature (1). This new and simple synthesis of OXI also verified the normal, cyclic structure of sodium kojate (CXIII). Moreover, the mild conditions of this synthesis recommended its use for the preparation of other derivatives of kojic acid from anhydrous sodium kojate and reactive organic halides.

The formation of CXI at lower alkali concentrations $(\mathrm{g})$, and its absence from the products of benzoylation in solutions of higher alkalinity ( $f$ ), might be taken to indicate a predominance of the "diketo" form of the kojate anion (LXIV) under the latter conditions. This assumption was advanced earlier in the discussion of the methylations.

The substance of m.p. $120-121^{\circ}$ had the composition $\mathrm{C}_{2} 7^{\mathrm{H}_{20}} \mathrm{O}_{8}$, which was that of a tribenzoate of the expected enolic cleavage product of kojic acid. By analogy with the already quoted work of Willstätter and Pummerer (125) on $\gamma$-pyrone (XCV to XCIX), an enolic tetrabenzoate (CXIV) should have been formed. Such analogies could, of course, only be drawn with certain reservations. The two most probable structures for an enolic tribenzoate of kojic acid were CXV and CXVI. Both of these structures contained two carbonyl groups, and it was obviously desirable to confirm their presence by the preparation of oximes, or substituted hydrazones. However, an unforeseen difficulty was encountered in the extreme lability of the "tribenzoate" under certain conditions. From its solutions in hydrocarbon solvents, and in glacial acetic 


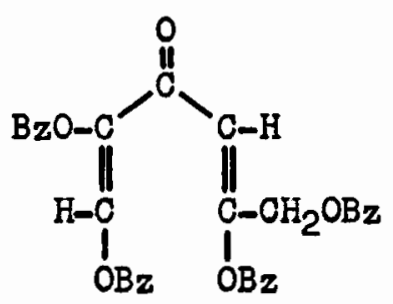

CXIV

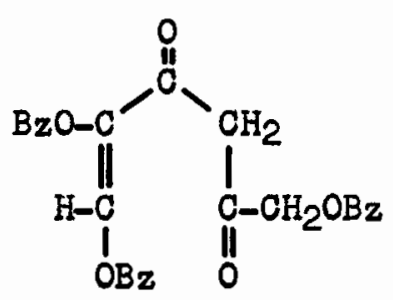

CXV<smiles>COC(=O)C(C(=O)O)C(=O)C=CC(=O)O</smiles>

CXVI<smiles></smiles>

CXVII

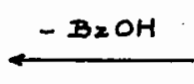

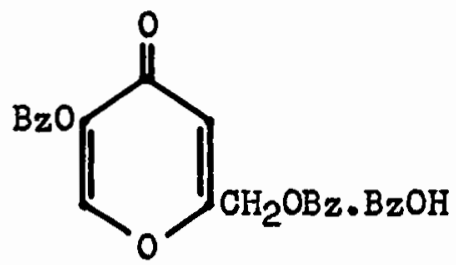

CXVIII

acid, the "tribenzoate" could be crystallized without change; but it could not be recovered from its solutions in most polar liquids, e.g. acetone, dioxane, alcohol, or their mixtures with water. Equimolecular quantities of dibenzoylkojic acid (CXVII) and benzoic acid were obtained in the latter cases (m). This result was first attributed to a great ease of hydrolysis of the enolic ester linkage, and to the instantaneous cyclization of the free enol to give CXVII. Consequently, it was not surprising that in all further efforts to prepare a characteristic derivative of the "tribenzoate", compounds derived from CXVII were obtained instead (m).

For example, the product from attempted condensations of the "tribenzoate" with hydroxylamine hydrochloride, as well as with benzoylhydrazine, contained no nitrogen, and was identified a monobenzoate of kojic acid, an isomer of CXI (m). The same monobenzoate was also produced, under identical conditions, from dibenzoylkojic acid (CXVII)(n). Attempts to hydrogenate the olefinic linkage of the "tribenzoate" (CXV, 
CXVI) over a platinum catalyst yielded inconclusive results (m), and so did the catalytic hydrogenation of CXVII (u). Attempts to achieve reduction of the "tribenzoate" with zinc in hot glacial acetic acid (m) led to reductive fission of an ester linkage, with the production of benzoylallomaltol, which was also obtained by applying the same reaction to CXVII $(v)$. Both of these reactions, the preferential hydrolysis by hydroxylamine, and the preferential cleavage with zinc in acetic acid, were studied in detail with dibenzoylkojic acid (CXVII) and will be discussed separately in two subsequent sections.

These results explained why Yabute (3) had failed to isolate the "tribenzoate" when he benzoylated kojic acid (II) in aqueous alkali; he crystallized the crude product from othanol. Neither did Traetta Mosca (5) obtain any product other than dibenzoylkojic acid (CXVII) from the benzoylation of II in pyridine; he too used ethanol for crystallization. By the use of anhydrous conditions and of hydrocarbon solvents it was possible to obtain a small amount of the "tribenzoate" together with CXVII from II and an excess of benzoyl chloride in pyridine $(h)$.

It seemed most unlikely that a true valence bond in an ester group could be ruptured with the apparent ease displayed in the examples cited, even allowing for the known lability of enolic ester linkages. The rapid and complete dissociation of the "tribenzoate" in polar solvents, in particular, suggested bonding of a much weaker kind, e.g. through van der Wals forces. The composition of the "tribenzoate" was identical with the sum-composition of dibenzoylkojic acid (CXVII) and benzoic acid. The conclusion that the supposed enolic tribenzoate was actually an addition compound (CXVIII) formed from dibenzoylkofic acid (CXVII) 
and benzoic acid was very convincingly supported by comparing the infrared spectrum of CXVIII with that of an equimolecular mixture of the two components. Both spectra were measured on benzene solutions of the respective compounds by Mr. A. W. Pross of the Central Research Laboratory, Canedian Industries (1954) Limited, McMasterville, Quebec, whose help we gratefully acknowledge. The two spectra, shown in Figure 2, were identical over the whole range of measurement. Hunter and his co-workers (127) studied the absorption spectre of severel types of molecular addition compounds in the ultraviolet and visible regions; the absorption plot of the addition compound was in all cases a mere superposition of the plots of the two components measured individually. The van der Warls forces which held together the component molecules of CXVIII consisted most likely of a mutual attraction of two dipoles. A closer study of these forces and of the structure of CXVIII was beyond the scope of this work, and would have required elaborate crystallographical and physicochemical investigations.

The recognition of the so-called "tribenzoate" as a molecular addition compound (CXVIII) helped to account for some of the details of the benzoylation reactions. For instance, the higher yield of CXVIII, and the absence of dibenzoylkojic acid (CXVII) from the products, in the benzoylation carried out at the lower alkali concentrations ( $g$ ) was probably caused by the presence of a considerable amount of free benzoic acid in the liquor. Similarly, in pyridine (h) some benzoyl chloride might have been slowly hydrolysed to benzoic acid, which then formed CXVIII by addition to CXVII.

Although it could not be considered as final proof, the failure 
Fig. 2: Infra-Red Spectrum of the Molecular Addition Compound of Dibenzoylkojic Acid and Benzoic Acid (Top), end of an Equimolecular Mixture of Dibenzoylkojic Acid and Benzoic Acid (Bottom), Measured in Benzene Solution. 


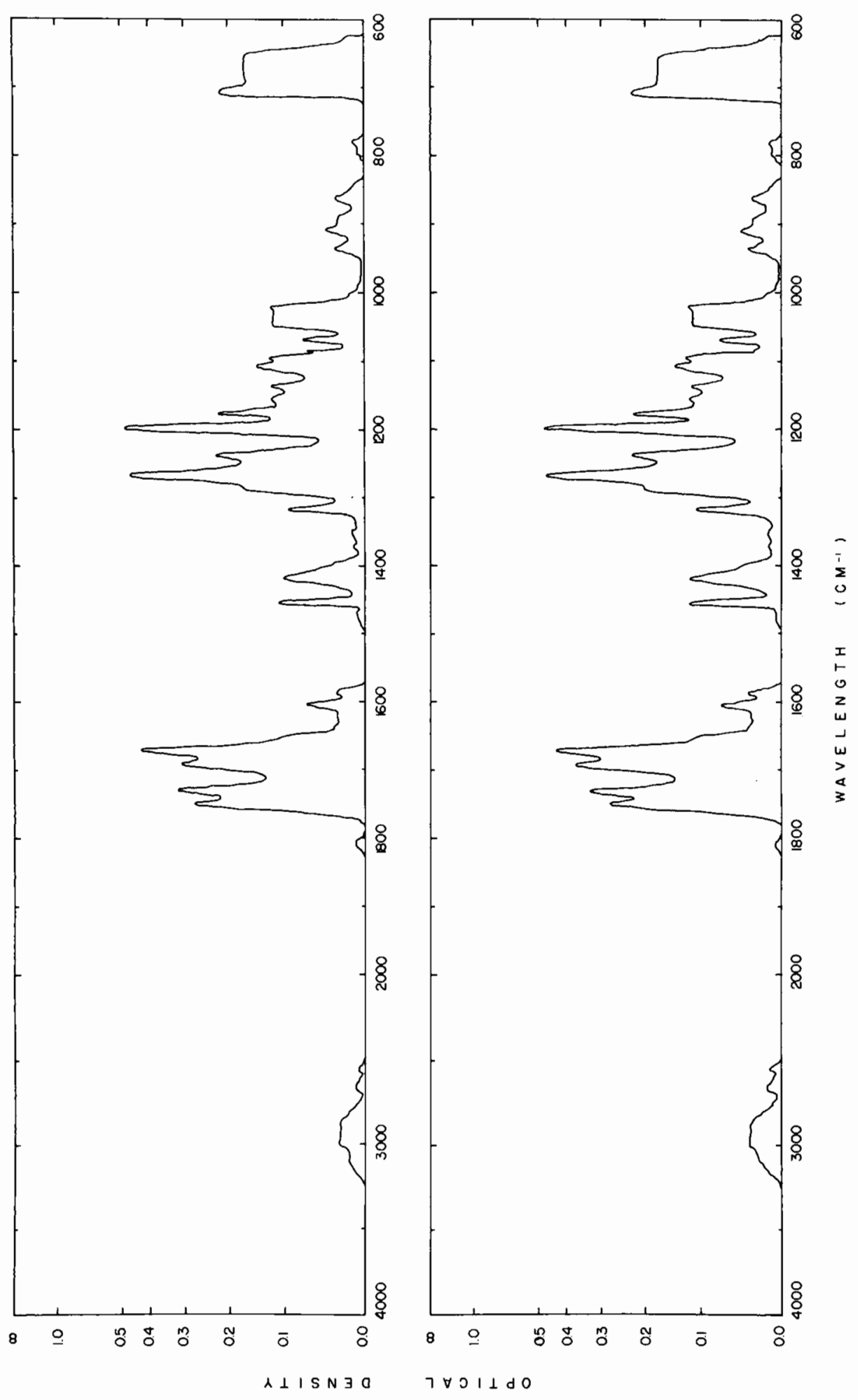


to isolate open chain enolic benzoates from these reactions constituted good ovidence that kojic acid did not suffer ring cleavage in aqueous alkali under the conditions of the benzoylations. These results supported the conclusions tentatively draw from the methylation reactions.

Debenzoylation of 5-0-Benzoyl Derivatives of Kolic Acid by Hydroxylamine

Preferential Hydrolysis of Dibenzoylkojic Acid

The production of a new monobenzoate of kojic acid was observed in a reaction between the so-called "tribenzoate" of kojic acid and hydroxylamine hydrochloride in pyridine. When the "tribenzoate" was recognized as an addition compound (CXVIII), further studies of this reaction were conducted with dibenzoylkojic acid (CXVII). The same monobenzoate was formed when CXVII and pyridine were heated in alcohol, and when pyridine was replaced by sodium acetate $(n)$. The most satisfactory procedure, however, was to dissolve CXVII and two equivelents of hydroxylamine hydrochloride in pyridine at room temperature, and to allow the reaction to continue for twelve hours. The almost pure product was recovered in $90 \%$ yield by diluting the pyridine solution with cold water, and collecting the crystalline precipitate on a filter. The pure monobenzoate (CXIX) melted at $180-181^{\circ}(n)$.

Since benzoylation of CXIX gave dibenzoylkojic acid (CXVII) (0), no other change than hydrolysis had occurred in the formation of CXIX from CXVII. The wine color produced by CXIX with ferric chloride indicated the presence of a phenolic hydroxyl group, and the site of the 
hydrolysis was thereby revealed. These data alone made it quite certain that the new monobenzoate was 2-(benzoxymethyl)-5-hydroxy- 8 -pyrone (CXIX). To confirm this structure, CXIX was methylated with diazomethane to give 2-(benzoxymethyl)-5-methoxy- $\gamma$-pyrone (CXX), a crystaline solid of m.p. $110-111^{\circ}(p)$. The proof was completed by also preparing CXX from 2-(hydroxymethyl)-5-methoxy- $\gamma$-pyrone (CV) and benzoyl chloride in pyridine $(q)$. The correctness of the position of the methoxy group was assured by the original synthesis of $O V$ from kojic acid (II) with diazomethane $(7,9,10)$<smiles>O=c1ccoc([18OH])c1</smiles>

CXVII<smiles>O=c1ccocc1OCc1ccccc1</smiles>

CXIX

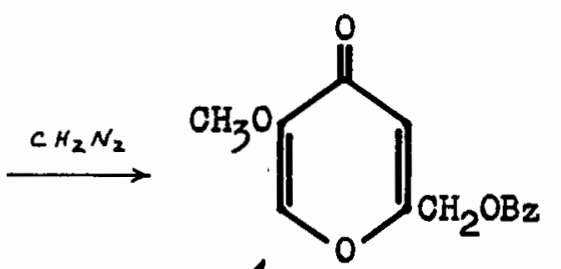
$\operatorname{cxx}$<smiles></smiles>

ov

The reaction between esters and hydroxylamine in the presence of bases is now a standard method for the preparation of hydroxamic acids. Tiemann and Krïger (128) discovered in 1885 that hydroxylamine, liberated from its hydrochloride by alkali, cleaved ethyl benzoate to give benzohydroxamic acid. The reaction was allowed to continue for several days at $100^{\circ}$ in a closed ressel; the yield was poor. Jeanrenaud (129) 
improved the procedure, and made it suitable for the synthesis of hydroxamic acids in general. He used one mole of hydroxylamine hydrochloride and two moles of sodium ethoxide for each mole of ester, conducted the reaction at room temperature, and reached yields of $40 \%$. Jeanrenaud's method formed the basis of many recent preparative procedures, among them the synthesis of benzohydroxamic acid given in Organic Symtheses (130). In some cases the cleavage of esters was also achieved with free hydroxylomine (131), but generally the reaction wes slow in the absence of alkaline reagents (132).

In all the instances mentioned, the purpose of the reaction was to obtain the hydroxamic acid derived from the acid component of the ester, and the procedures were perfected to the point where yields of $60-80 \%$ could be reached. The cleavage of the "phenolic" ester linkage of dibenzoylkojic acid (CXVII) by hydroxylamine was essentially the same reaction 8.s that observed by Tiemann and Krüger (128), but the purpose was exactly the opposite; here the alcohol component of the ester was the product sought, and obtained in very high yield. The reaction had apparently never been used to serve this end; a search of the literature revealed only one case analogous to the debenzoylation of CXVII.

Ingold and Shoppee (133) observed that the oximation of 2-benzoxy4,4,5,5-tetramethyl-2-cyclopenten-1-one (CXXI) with four moles of hydroxylamine hydrochloride and four moles of anhydrous sodium acetate in hot absolute ethanol was accompanied by debenzoylation to give the mono-oxime of $3,3,4,4$-tetramethyl-1,2-cyclopentanedione (CXXIII). Under identical conditions the fully saturated analog of CXXI (CXXIV) reteined its benzoyl group to give 2-benzoxy-4,4,5,5-tetramethylcyclopentanone 


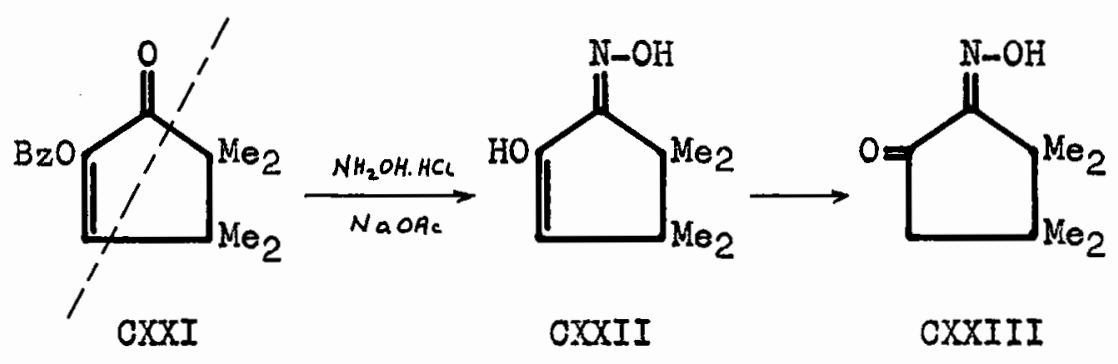

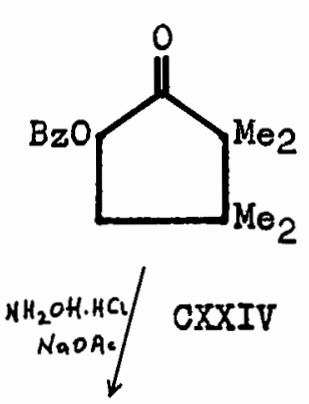

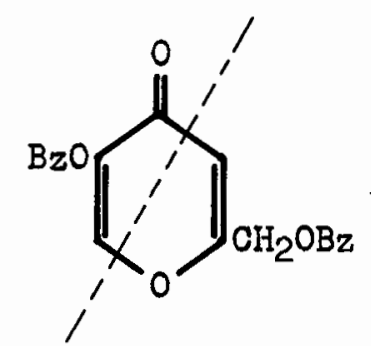

CXVII

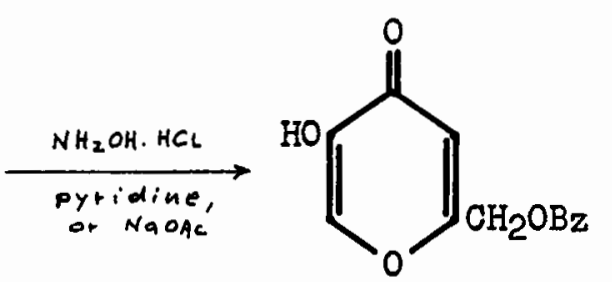

CXIX<smiles>O=[W]C1CC([N+](=O)[O-])C1C(=O)O</smiles>

CXXV

oxime (CXXV). The similarity of these reactions to the behaviour of dibenzoylkojic acid (CXVII) is striking: in the molecules of CXXI and CXVII the portions above the dotted Iine are identical; the ester linkage that was cleaved was enolic in CXXI, and the primary product (CXXII) rearranged to the stable keto form (CXXIII). In CXVII the ester linkage cleaved was phenolic, and in consequence the primary product was also the final, stable product (CXIX). No oximation occurred with CXVII, because the carbonyl group participated in the resonance system of the $\gamma$-pyrone nucleus; no such resonance system existed in CXXI. The alcoholic ester linkage of CXVII was practically unaffected by hydroxylamine, as was the alcoholic benzoate group of CXXIV.

Ingold and Shoppee (133) were also the first to use the weak base sodium acetate in place of alkali or alkali alcoholates in this cleavage reaction. The isolation of benzoic acid as a by-product suggested that the use of sodium acetate led to simple hydrolysis, rather than to the formation of a hydroxamic acid. They demonstrated the hydrolytic effect 
of hydroxylamine and sodium acetate on simpler substances also; phenyl benzoate was cleaved in hot absolute ethanol to give $20 \%$ of benzoic acid and phenol. Sodium acetate alone had no effect on phenyl benzoate (133); neither did it attack dibenzoylkojic acid $(n)$.

In the present work the use of pyridine, both as solvent and as the weak base taking the place of sodium acetate, permitted a choice of conditions milder then those of the experiments cited. As a result, the alcohol component of the ester cleaved by hydroxylamine was recovered nearly quantitatively. The fate of the acid component was of little interest in this case, and was not ascertained.

The most interesting feature of the cleavage was its selectivity. Hydrolysis apparently occurred smoothly when the enolic or phenolic ester group was adjacent to a carbonyl group; under identical conditions the cleavage of simple phenolic ester linkages was slow and alcoholic esters were unaffected.

Selective Debenzoylation of Other 5-Benzoates of Kojic Acid

The following experiments were to discover whether linkages weaker than benzoate, and located in the side chain of kojic acid, would remain

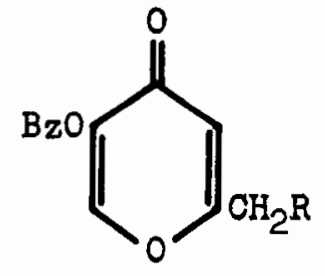

CXXVI, $R$ = Acetoxy

CXXVIII, $R=$ Triphenylmethoxy $\operatorname{CXXX}, R=H$

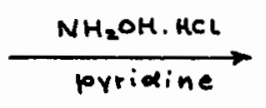<smiles>[R2]c1cc(=O)cco1</smiles>

CXXVII, $R=$ Acetoxy

CXXIX, $R=$ Triphenylmethoxy CXXXI, $\mathrm{R}=\mathrm{H}$ 
unaffected while the phenolic benzoate group was cleaved by hydroxylamine. The results gave an affirmative answer: 2 -(acetoxymethyl)-5benzoxy- $\gamma$-pyrone (CXXVI) was hydrolysed to 2-(acetoxymethyl)-5-hydroxy$\gamma$-pyrone (CXXVII) in 83\% yield ( 8 ), and 5-benzoxy-2-(triphenylmethoxymethyl) $-\gamma$-pyrone (CXXVIII) to 5-hydroxy-2-(triphenylmethoxymethyl)- $\gamma-$ pyrone (CXXIX) in $66 \%$ yield $(t)$. The conditions of these hydrolyses were those applied to dibenzoylkojic acid, and consisted of solution for twelve hours at room temperature in pyridine containing an excess of hydroxylamine hydrochloride. Another example of the general applicability of the reaction was the hydrolysis of benzoylallomaltol (CXXX) to allomaltol (CXXXI) in $71 \%$ yield. ( $w$ ).

When applied to kojic acid, one of the uses of the reaction under discussion would be in the preparation of derivatives of the alcoholic hydroxyl group. A search of the literature revealed that only three such derivatives were known (Historical Introduction, Table II), of which only two were prepared directly. This lack was largely caused by the greater reactivity of the phenolic hydroxyl group. The simplest case of this use of the selective debenzoylation reaction was the preparation of 2-(benzoxymethyl)-5-hydroxy- $\gamma$-pyrone (CXIX) in two steps: benzoylation of kojic acid in dry pyridine gave a $98.5 \%$ yield (i) of dibenzoylkojic acid (CXVII), which was debenzoylated to CXIX in a yield of $90 \%$ $(n)$, the overall yield thus being $88.5 \%$.

The preparation of all other derivatives of the alcoholic hydroxyl group would have to start from 5-benzoxy-2-(hydroxymethyl) $-\gamma$-pyrone (CXI), now readily available from anhydrous sodium kojate (1), and capable of easy substitution in the side chain by a variety of groups. 
Thus acetylation of CXI gave CXXVI $(r)$, which was debenzoylated to CXXVII (s); the yields in the three simple steps of the synthesis of CXXVII were $79.5,93$ and $83 \%$ respectively, or $61.5 \%$ overall. Careful adjustments in individual ceses could no doubt raise the yield somewhat. The reaction might also be of use in the flavonol series (CXXXII) after the hydroxyl group in position 3 had been protected by benzoylation.

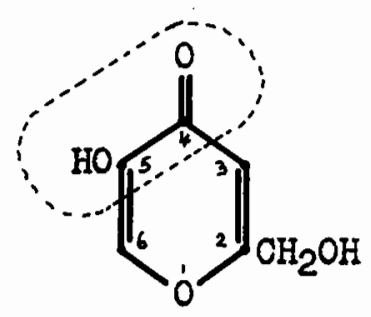

II

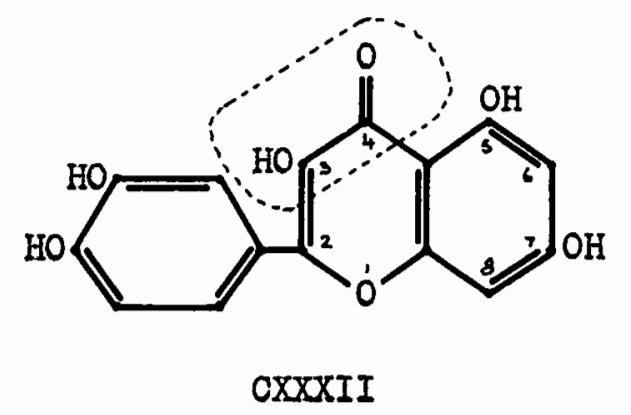

CXXXII

After effecting any desired change at other points of the molecule, the protecting benzoyl group could be removed easily under mild conditions by hydroxylamine hydrochloride in pyridine. This prediction is based on the identity of the encircled portion of the molecule of flavonols with the corresponding portion of the molecule of kojic acid (II). Without doubt hydroxylamine could be used to effect the preferential hydrolysis of 5-0-acetyl derivatives of II, or of 3-0-acetyl derivatives of cXXXII, extending thereby the scope and usefulness of the reaction.

\section{Reduction of Dibenzoylkojic Acid}

Hydrogenation over Platinum

The attempts to reduce the molecular compound of dibenzoylkojic 
acid and benzoic acid, then believed to be an open chain enolic tribenzoate of kojic acid, indicated that dibenzoylkojic acid (CXVII) was susceptible to certain reducing agents. One of these was the combination of hydrogen and platinum. The experiment was repeated with dibenzoylkojic acid (CXVII) itself; the catalyst was reduced platinum, the solrent glacial acetic acid, and the hydrogenation was carried out at room temperature and atmospheric pressure $(u)$. Although the hydrogen uptake had not yet come to a halt, the reaction was stopped after the absorption of 7.4 atoms per molecule of dibenzoylkojic acid. The products were a sirup together with approximately 0.6 moles of benzoic acid. The sirup could not be crystallized, and its investigation was abandoned in view of the inconclusive results obtained by Armit and Nolan (10) in their studies of the hydrogenation of kojic acid derivatives over palladium. The production of benzoic acid might have been caused by a hydrolytic cleavage, or by hydrogenolysis. The hydrolysis of esters by catalysts for hydrogenation is not commonly observed. Although Verkade and co-workers (134) reported the hydrolysis of a di---benzoyl derivative of glycerol over platinum oxlde in alcohol, Miescher and Scholz (135) attributed Verkade's result to the presence of a trace of alkali in the catalyst; the acidic medium of the present hydrogenation would eliminate this possibility. The likelihood of a cleavage by hydrogenolysis seemed somewhat greater, for the side chain of kojic acid was in many ways comparable to that of benzyl alcohol. The latter compound, its ethers and its esters, all underwent hydrogenolysis very readily on catalytic hydrogenation to give toluene as the product arising from the benzyl alcohol portion of the molecule (136). In the case of the catalytic 
hydrogenation of dibenzoylkojic acid (CXVII), the evidence was insufficient to reach a decision, but the results of the reduction with zinc in acetic acid, now to be discussed, favored hydrogenolysis.

Reductive Fission by Zine in Glacial Acetic Acid

When a solution of dibenzoylkojic acid (OXVII) in glacial acetic acid was heated with a large excess of zinc dust for 2.5 hours on the steam bath $(v)$, the products were $32 \%$ of a crystalline substance melting at $124.5-126^{\circ}$, and nearly one mole of benzoic acid per mole of CXVII. The analytical sample of the first product melted at $126-127^{\circ}$, and its composition agreed well with that of a dibenzoate of tetrahydrokojic acid; the production of benzoic acid was attributed to hydrolysis in an undesirable, competing side reaction. Debenzoylation of the reduction product by the hydroxylamine method gave $71 \%$ of a white crystalline solid melting at $150-151^{\circ}$ (w), which was smoothly reconverted to the original substance by benzoylation $(x)$. The debenzoylated product, however, still had a phenolic character, and a carbon content which was considerably lower than would have been required by the composition of a monobenzoate of tetrahydrokojic acid. Among the phenolic derivatives of kojic acid, allomaltol (CXXXI) had the same composition as the debenzoylated reduction product. While Yabuta (8) listed the melting point of allomaltol (CXXXI) as $166^{\circ}$, it was given recently by Looker and Okamoto $(76)$ as $151^{\circ}$. The analytical results of the latter workers were closer to the theoretical composition of CXXXI than were those of Yabuta. Allomaltol (CXXXI) was synthesized from 2-(chloromethyl)-5-hydroxy- 
$\gamma$-pyrone (CXXXII) by reduction with zinc in glacial acetic acid. The method of Kipnis and his co-workers (77) was found satisfactory for the preparation of CXXXII; the reduction was carried out following Yabuta's (8) procedure, but the yield of CXXXI was very low. This authentic sample of allomaltol (CXXXI) melted at $150-151^{\circ}$, and admixture with the debenzoylated reduction product of dibenzoylkojic acid, melting at 150$151^{\circ}$, did not depress the melting point. The identity of the reduction product proper was thereby established, as benzoylallomaltol (CXXX).<smiles>O=C1C=COc2coc1c2</smiles>

CXVII<smiles>O=c1ccocc1</smiles>

CXXXII
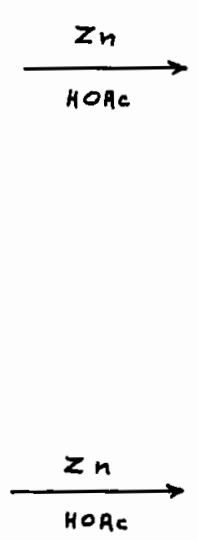

The melting point of the pure substance was $2^{\circ}$ lower than the one given by Yabute (8).

Thus the production of benzoylallomaltol (CXXX) from dibenzoylkojic acid (CXVII) was a case of reductive fission, and the recovery of close to one equivalent of benzoic acid as a by-product was explained. The nucleus of kojic acid, like that of the benzyl group, was resistant under the conditions of the reduction. This reductive fission was 
noteworthy because it had been achieved by using zinc in glacial acetic acid at the temperature of the steam bath. Benzyl esters are usually cleaved by means of molecular hydrogen and catalyst; chemical reducing agents have been used less frequently. The use of zinc for this purpose wes not listed in a recent review of the debenzylation reaction (136). Zinc has, however, been employed to reduce alcohols, primary (137), secondary (138) and tertiary (139) alike, to the corresponding hydrocarbons. In all three instances, the alcoholic hydroxyl group reduced was alphe to an aromatic ring, and probably had a reactivity not unlike that of the primary hydroxyl group in kojic acid.

\section{Condensations of Kojic Acid and Its Derivatives with Carbonyl Reagents}

\section{Analogous Reactions of Other $\gamma$-Pyrones}

Only two major groups of carbonyl reagents will be considered in this section: the substituted ammonias $\left(\mathrm{RNH}_{2}\right)$, e.g. aniline and hydroxylamine; and the substituted hydrazines $\left(\mathrm{RNHNH}_{2}\right), e . g$. semicarbazide and phenylhydrazine.

With the exception of a few isolated cases, these reagents were not known to condense directly with the carbonyl group of the nucleus of $\gamma$-pyrones $(66,96)$. One of these exceptions was the formation of the p-nitrophenylhydrazone of diethyl chelidonate (CXXXIII, R = p-Nitrophenyl)* (140). Rather, when reaction did take place, the ring of the $\gamma$-pyrone

* Formulae CXXXIII to CXI are only schematic representations, and the substituents on the pyrone ring are not indicated. 


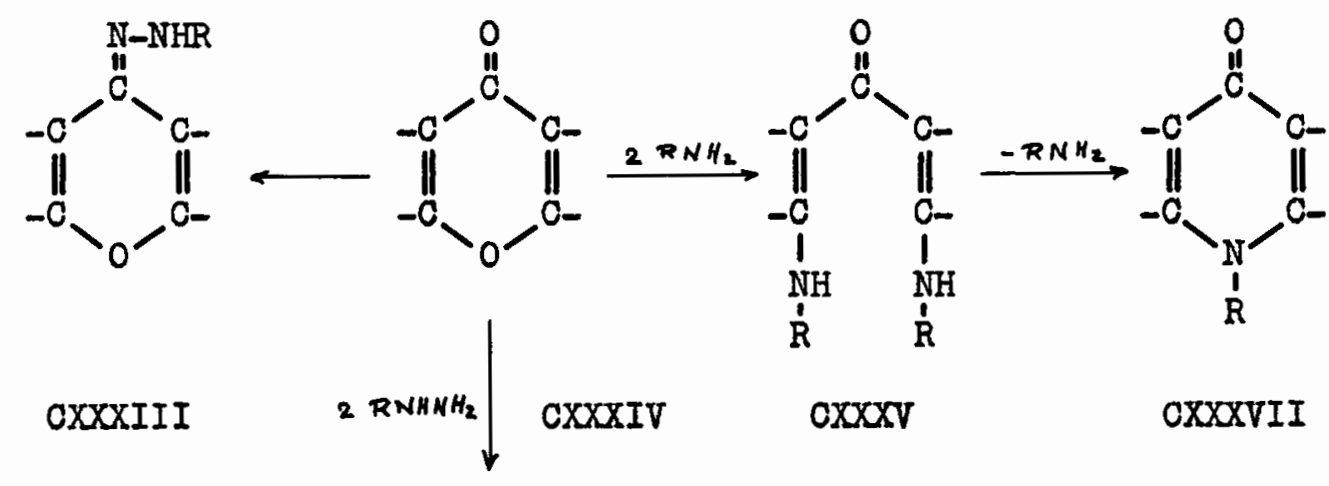

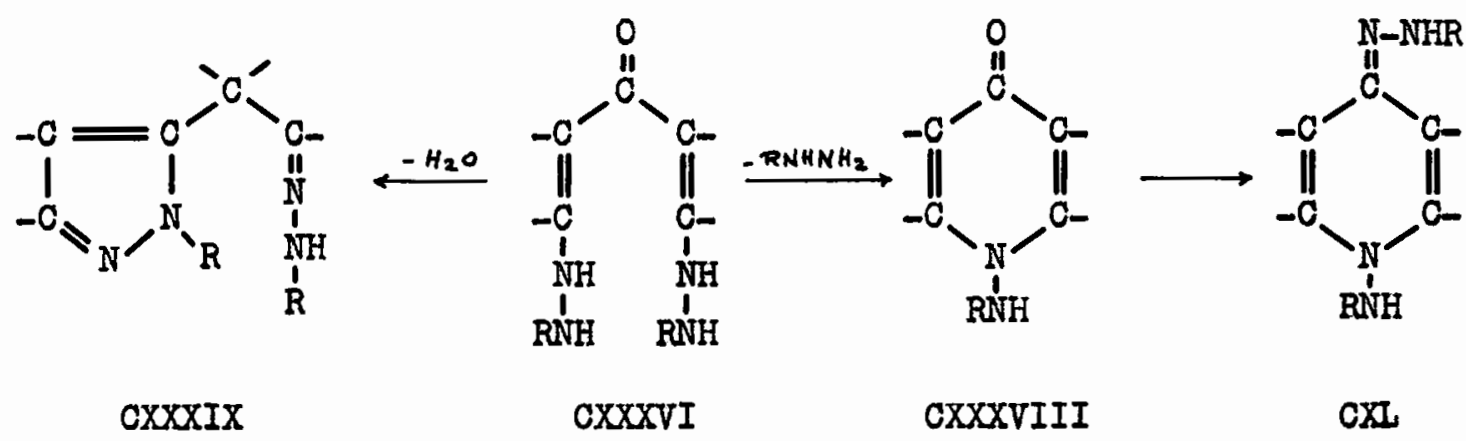

(CXXXIV) was cleared, and one molecule of the carbonyl reagent condensed with each of the two enolic hydroxyl groups created by the cleavage. The open chain products (CXXXV, CXXXVI) were practically always labile, and beceme stabilized through cyclization. Consequently, hardly any of these open chain intermediates were ever 1solated; an exception was the crystalline dianilide of bis-(hydroxymethylene)-acetone (CXXXV, $R$ = Phenyl), prepared by Borsche and Bonacker (141).

Cyclization was possible in two ways: with the loss of one of the groups derived from the carbonyl reagent to give N-substituted pyridones (CXXXVII, CXXXVIII); and with condensation between one of the new groups and the carbonyl group in the $\beta$-position, the original carbonyl group of the pyrone. The second mode of cyclization was observed most frequently with the intermediates of structure CXXXVI, and resulted in the formation of pyrazole derivatives (CXXXIX). N-Substituted pyridones of 
structure CXXXVII were prepared from $\gamma^{\wedge}$-pyrones and hydroxylamine (142), aniline (141) and semicarbazide (143); those of structure CXXXVIII from $\gamma$-pyrones and p-nitrophenylhydrazine (140). It was also claimed that some $\gamma$-pyrones condensed with two molecules of p-nitrophenylhydrazine to give as a final product the p-nitrophenylhydrazone of an N-p-nitroanilinopyridone (CXL, $R=p$-Nitrophenyl) (140). The formation of pyridone derivatives in the latter case was questioned by Ainsworth and Joneo (144), who studied a number of derivatives with the pyrazole structure (CXXXIX) prepared from $\gamma$-pyrones and hydrazine or phenylhydrazine. They demonstrated that one of the derivatives described as having structure CXI (140) was actually a pyrazole with structure CXXXIX.

\section{Negative Regults with Some Carbonyl Reagents}

Customary conditions were adopted in all attempts to condense carbonyl reagents and kojic acid derivatives. The temperatures ranged from 25 to $100^{\circ}$, the pressure was always atmospheric and no drastic reagents were used.

Most of the experiments with negative results have been discussed from a different point of view in previous sections. Dibenzoylkojic acid (CXVII), 2-(acetoxymethyl)-5-benzoxy- 8 -pyrone (CXXVI), 5-benzoxy-2-(triphenylmethoxymethyl) $\gamma$-pyrone (CXXVIII), and benzoylallomaltol (CXXX) all failed to form an oxime under the usual conditions of oximation $(n, s, t, w)$. No benzoylhydrazone was formed when dibenzoylkojic acid (CXVII) was brought together with benzoylhydrazine in glacial acotic acid (m). When dibenzoylkojic acid (CXVII) was added to a 2,4-dinitro- 
phenylhydrazine solution containing some sulfuric acid, no 2,4-dinitrophenylhydrazone was formed; only a mixture of unchanged CXVII and 2,4dinitrophenylhydrazine was recovered from the solution after dilution with water. When kojic acid or its 5-benzoate were bolled with semicarbazide hydrochloride and pyridine in ethanol, in both cases the only water-insoluble product isolated was a small quantity of biurea, the high-melting autocondensation product of semicarbazide itself.

Open Chain Semicarbazones Derived from Dibenzoylkojic Acid

Although dibenzoylkofic acid failed to condense with benzoyl- and 2,4-dinitrophenyl-hydrazine, under the same conditions a condensation with semicarbazide formed a water-insoluble product. Dibenzoylkojic acid (CXVII), three equivalents of semicarbazide hydrochloride, and a somewhat larger excess of pyridine were suspended in $90 \%$ ethanol, the mixture was heated to boiling, and the resulting clear solution was kept at the temperature of reflux for fifty minutes $(y)$. Yields of slightly over $40 \%$ of a microcrystalline yellow-white substance were obtained from these reactions. The product was insoluble in practically all liquids, except glacial acetic acid, in which it dissolved sparingly. Becaure of this poor solubility the substence could be boiled freely with solvents to remove impurities. Thus water was used to extract hydrochlorides, and dioxane or ethyl acetate to extract any unused starting material and byproducts. If the crude solid was powdered prior to these extractions, the final product was practically analytically pure, melting at $212^{\circ}$ with decomposition, and could be used for further reactions. The 
analytical sample, melting at $215^{\circ}$ with decomposition, was purified, after the extractions, by reprecipitation with water from its solution in glacial acetic acid. Care had to be taken not to heat the substance in glacial acetic acid above seventy degrees and so cause its degradation. Concentration of the acetic acld solution in racuo was also accompanied by extensive degradation.

This high-melting substance was neutral and had a composition corresponding to $\mathrm{C}_{22} \mathrm{H}_{22} \mathrm{~N}_{6} \mathrm{O}_{7}$. Assuming that the reaction between dibenzoylkojic acid and semicarbazide had taken the usual course, the primary product would have been an open chain derivative (CXII), analogous to CXXXVI. It was evident that this intermediate had not undergone cyclization, because the composition of a cyclic derivative analogous to CXXXIX or CXI would have to be $\mathrm{C}_{22} \mathrm{H}_{22} \mathrm{~N}_{6} \mathrm{O}_{7}$ minus $\mathrm{H}_{2} \mathrm{O}$. Nevertheless, the intermediate must have changed, because the two secondary amine groups of structure CXII were not compatible with the insolubility of the new substance in dilute mineral acids. A shift to the corresponding disemicarbazone structure (CXIII) might have taken place.

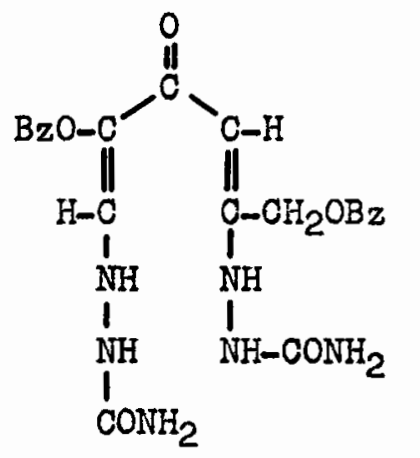

CXII<smiles></smiles>

CXLII

A small yield of an isomer of this open chain "disemicarbazone" of 
dibenzoylkojic acid was isolated by using a slightly modified procedure for the preparation $(z)$. The new isomer, a white crystalline solid melting at $172-172.5^{\circ}$ with decomposition, was nomed dibenzoylkojic acid $\beta_{-}$ "disemicarbazone", to distinguish it from the high-melting $\alpha$-isomer. The decision was made to confine further investigations to the $\alpha$-isomer, which will be referred to as the $\alpha$-"disemicarbazone" (CXIII) in the following discussion. Formula CXII should be considered chiefly as an aid to memory, and not as a proven structure.

In order to learn whether CXIII was a true disemicarbazone, the $\alpha$-isomer was treated with nitrous acid, one of the standard reagents for the fission of semicarbazones (145). The customary procedure had to be modified somewhat on account of the insolubility of the substance, and a concentrated aqueous solution of sodium nitrite was added, with constant stirring, to a suspension of CXIII in giacial acetic acid. As the reaction progressed at room temperature, more and more of CXIII went into solution. The results of the reaction depended on the excess of sodium nitrite used. With approximately sixteen equivalents of sodium nitrite the crude product was fairly uniform, and purification gave a white microcrystalline substance melting at $178-179^{\circ}$ with decomposition, in a yield of $69 \%(\mathrm{bb})$. When twenty-five equivalents of sodium nitrite were used, the crude product was rather heterogeneous, and seemed to be a mixture of several components. Only one of these could be isolated in the pure state as a white microcrystalline substance melting at $215.5^{\circ}$ with decomposition (a).

Analyses showed that these two products had an identical composition corresponding to $\mathrm{C}_{21} \mathrm{H}_{1} \mathrm{~N}_{3}{ }^{\mathrm{O}} 7$. The presence of three atoms of nitrogen per 
molecule suggested that only one of the two semicarbazone groups had been cleaved, the high proportion of carbon indicated that no benzoate group had been hydrolysed, and, again from the composition, it was quite certain that no cyclization had occurred in the process. For the sake of convenience the high-melting isomer will be referred to as dibenzoylkojic acid $\alpha$-"monosemicarbazone", and the low-melting isomer as dibenzoylkojic acid $\beta$-"monosemicarbazone". In this case the $\beta$-isomer was the more readily accessible, and was therefore chosen for further investigations.

The $\alpha$-"monosemicarbazone" might have been produced by the action of nitrous acid on the $\beta$-"monosemicarbazone", in which case the two compounds could be tautomers or geometric isomers, but not position isomers. If, however, the $\alpha$-monosemicarbazone" arose directly from the $\alpha$ - "disemicarbazone" by a different process from that resulting in the B-"monosemicarbazone", the possibility of position isomerism would also have to be considered. The second alternative was favored when the $\beta$-monosemicarbazone" was found to be practically unaffected by excess nitrous acid (bb).

The fact that only one semicarbazone group of dibenzoylkojic acid $\alpha$-disemicarbazone" (CXLII) was cleaved by nitrous acid indicated that the other semicarbazone group was elther stable enough to resist cleavage, which was rather improbable, or that it did not have a semicarbazone structure at all. As already mentioned, alternative structures that involved cyclization with loss of the elements of water were excluded by the composition of the mono- and di-semicarbazones. It was, however, conceivable that the molecule of the $\alpha$ - disemicarbazone" became 
stabilized by a tautomeric shift of one of the three double bonds depicted in formula CXIII. A conjugated system would thereby be created, which would derive further stability through hydrogen bonding. This concept is illustrated in formulae CXIIII and CXIIV, in which the structures are slightly distorted to make comparison with formulae CXII and CXIII easier. In this event, one of the semicarbazone groups would be expected to be more stable than the other.

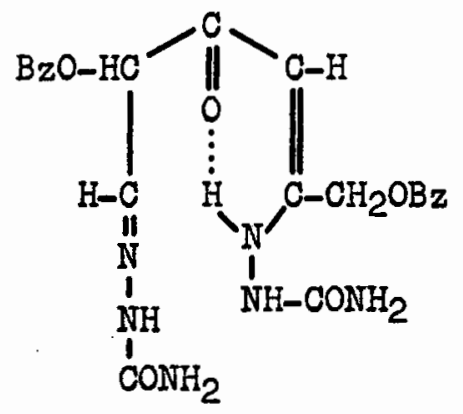

CXIIII

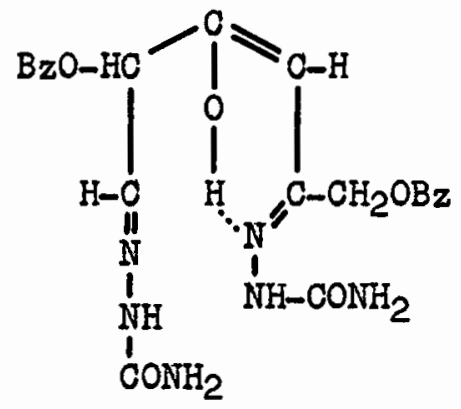

CXIIV

Attempts to acetylate the $\alpha$-"disemicarbazone", in order to demonstrate the presence of a secondary amino group, or of an enolic hydroxyl group, failed. In the reaction with nitrous acid leading to the "monosemicarbazones" no nitrosation occurred. Furthermore, the $\alpha$-"disemicarbazone" did not dissolve in hot or cold dilute mineral acids and did not give a color with ferric chloride. The first two reactions are usually given by secondary amines, the third by enolic compounds. Although these tests might have been hindered in the present case by the insolubility of the $\alpha$-disemicarbazone", their results must be considered as adverse to structures CXIIII and CXIIV.

A certain amount of information about the structure of the dibenzoylkojic acid $\alpha$-"disemicarbazone" was obtained from degradation reactions. 
A treatment with boiling $10 \%$ aqueous hydrochloric acid gave a small yield of a yellow-white microcrystalline solid, melting at $244-244.5^{\circ}$, and nearly one equivalent of benzoic acid (cc). From analyses and a molecular weight determination, the composition of the high-melting solid could be expressed by the empirical formula $\mathrm{C}_{21} \mathrm{H}_{15} \mathrm{~N}_{3} \mathrm{O}_{5}$. This composition indicated the retention of both benzoyl groups, the fission of one semicarbazone group, and the loss of two molecules of water, no doubt through cyclization. A closer study of this somewhat inaccessible substance could not be fitted into the present research. When treated with cold 10\% aqueous potassium hydroxide, both of the benzoate groups in the $\alpha$-"disemicarbazone" were smoothly saponified; although the recovery of benzoic acid was nearly quantitative, no other product could be recovered (dd). An oxidation with aqueous potassium permanganate produced low yields of benzoic and benzoylglycolic acids, but no pyrazole or pyridone carboxylic acids (ee), such as cyclic structures like CXXXIX or CXI would no doubt have yielded (144). The oxidation of dibenzoylkojic acid $\beta$-monosemicarbazone" by aqueous potassium permanganate also yielded benzoic and benzoylglycolic acids (ff).

Without exception, these results indicated the absence of a stable cyclic structure in the semicarbazones. The potential ability to undergo cyclization was demonstrated by the reaction with hot dilute acid. The benzoylglycolic acid produced in the oxidations arose most probably from carbon atoms 5 and 6 (see formula CXIII), and in this event was in agreement with structures CXIIII and CXIV for the disemicarbazone, but rendered CXIII very improbable.

An attempt to reconvert the $\beta-$ "monosemicarbazone" into a 
disemicarbazone by a treatment with semicarbazide hydrochloride and pyridine in boiling methanol failed, the starting material being recovered unchanged (gg). Hydroxylamine hydrochloride and pyridine in boiling methanol likewise had no effect on the $\beta$-"monosemicarbazone" (hh). These results were surprising, because the presence of a carbonyl group created by the fission of one of the semicarbazone groups in the "disemicarbazone". could reasonably have been assumed. The second of the two negative results also suggested that an enolic benzoate group was absent, since hydroxylamine hydrochloride was known to remove this group from dibenzoylkojic acid $(n)$.

Even at the close of this research, it was not found possible to propose definite structures for any of the four "semicarbazones" derived from dibenzoylkojic acid, but there was little doubt left that they were all open chain compounds. As such, their preparation had achieved two of the original aims of the research: the cleavage of kojic acid at position 1 without the simultaneous rupture of carbon-to-carbon bonds, and the condensation with carbonyl reagents. Unexpectedly, it was by efforts to accomplish the latter objective that success was gained in the former.

An obvious question remained to be answered why did kojic acid fail to undergo cleavage in normal aquous alkali, a medium so generally used to cleave $\gamma$-pyrones, when one of its derivatives was readily cleaved by the agency of a much weaker base, semicarbazide? An answer might be found by considering the distribution of electrons in kojic acid in the presence of reagents of differing character. The conventional structure of kojic acid (II) was found to be 
inadequate to explain all the reactions of the compound, as is generally true for $\gamma$-pyrones (66). A series of structures, intermediate between II and one of the extreme forms with separated charges (CXLV) would make contributions to the resonance hybrid of kojic acid in a neutral medium. Position 6 would thereby acquire electrophilic properties, and consequently an affinity for nucleophilic reagents. In an alkaline medium kojic acid would exist in the form of an anion, and carry a full negative charge; important contributions to the resonance hybrid of this anion would come from structures LXIII and LXIV, to which reference has been made previously. In these cases position 6 would acquire a nucleophilic character and would attract electrophilic reagents.

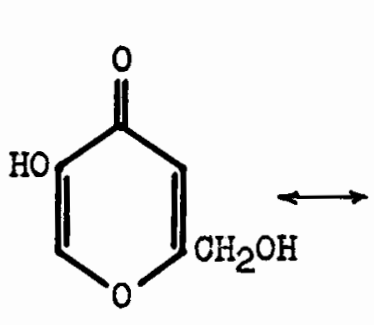

II

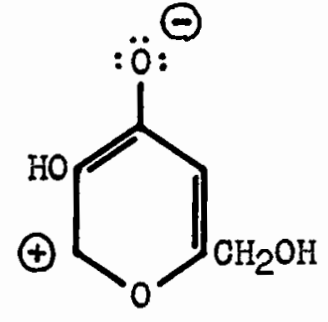

CXIV<smiles></smiles>

LXIII<smiles>O=C1C=C(O)C(=O)C(=O)O1</smiles>

LXIV

Cleavage of the $\gamma$-pyrone ring is presumably initiated at position 6 . The hydroxyl ion and semicarbazide are the two reagents under consideration, and both are nucleophiles. Semicarbazide, acting in an essentially neutral medium, would be able to initiate cleavage by a nucleophilic addition at position 6 . On the other hand, in the alkaline solution the hydroxyl ion would face another nucleophilic center at position 6; addition would fail to take place, and consequently no cleavage could occur (146). An increase in the alkali concentration might, however, cause the degradation of kojic acid through a weakening of the carbon-to-carbon 
90

bonds. This postulate is well supported by the fact that none of the $\gamma$-pyrone, whose cleavage by alkali to open chain compounds had been reported, contained a nuclear phenolic hydroxyl group (66,96).

Two ways seem to be open for further attempts to obtain open chain cleavage products from kojic acid: by dilute, aqueous alkali, after elimination of the undesirable effect of the kojate anion through the formation of an alkali-resistant derivative; and by weak bases similar to, but simpler in structure than, semicarbazide. 


\section{EXPERIMENTAL}

Weights and melting points were always determined after drying of the samples at suitable temperatures and reduced pressures, using chemical drying agents whenever advisable. The melting points were expressed in degrees centigrade, and were all corrected (147). Molecular weights were determined by the Rast camphor method. The infra-red spectra were observed on a Perkin Elmer Model 21 double-beam spectrophotometer, fitted with a rock salt prism, by $\mathrm{Mr}$. A. W. Pross of the Canadian Industries (1954) Limited Central Research Laboratories at McMasterville, Quebec. The combustions for the carbon and hydrogen analyses were carried out in a tube with "combination band" filling, according to Niederl and Niederl (148). Two carbon and hydrogen values were checked by Schwarzkopf Microanalytical Laboratories, Woodside, N. Y., who also carried out all nitrogen determinations. The analyses for aluminum and sodium were done by micro-ashing (149). Methoxyl analyses were by the standard procedure of Peniston and Hibbert (150), benzoyl determinations by the customary method for acetyl groups modified according to Meyer and Hart$\operatorname{mann}(151)$.

Solutions were evaporated in a rotary evaporator, unless otherwise stated; the temperatures indicated are those of the water bath surrounding the revolving flask which contained the solution. The ethanol used was commercial absolute grade, unless otherwise specified. 
(a) Action of Cold Aqueous Sodium Hydroxide on Kofle Acid

One gram $(0.00706$ mole $)$ of kojic acid was added to a solution of $0.56 \mathrm{~g} \cdot(0.0141 \mathrm{~mole})$ of sodium hydroxide in $11.3 \mathrm{ml}$. of water. The resulting yellow, clear solution of approximately pH 13 was stored for 13.5 hours at room temperature, and was then acidifled to $\mathrm{pH}$ 5-6 with $20 \%$ aqueous sulfuric acid. After being chilled, seeded with a few crystals of kojic acid, and stored for 12 hours at $-3^{\circ}$, the solution deposited white needles, weighing $0.77 \mathrm{~g}$. and melting at $152-153^{\circ}$. A mixed melting point determination established the identity of this substance with kojic acid. The mother liquor was diluted with a few ml. of water, and three fifths of its volume was distilled at atmospheric pressure. The distillate, when titrated with standard sodium hydroxide to the phenolphthalein endpoint, required $0.93 \mathrm{ml}$. of $0.07608 \mathrm{~N}$ sodium hydroxide, corrosponding to 0.000071 mole of formic acid (1\%).

In other experiments a small second crop of kojlc acid was gained by concentration of the mother liquor to one third to one half volume, seeding, and chilling before filtration. In these cases the distillation of formic acid was omitted.

(b) Preparation of Sodium Kojate

A solution of $3.00 \mathrm{~g} \cdot(0.0211 \mathrm{~mole})$ of $\mathrm{kojic}$ acid and $0.88 \mathrm{~g}$. $(0.0212$ mole) of sodium hydroxide (purity $97 \%)$ in $15 \mathrm{ml}$. of water was evaporated to dryness at $40^{\circ}$ and $15 \mathrm{~mm}$. pressure. The residual solid was recrystallized twice from about $5 \mathrm{ml}$. of hot water, purity rather than a high yield being the primary objective. The final product was 
dried on a filter by suction, to give $2.62 \mathrm{~g}$. of white crystals. For analytical purposes the samples of sodium kojate were dried in the air at $25^{\circ}$ to a constant weight. The amount of water of crystallization was determined in such samples by further drying to constant weight either at $110^{\circ}$ and atmospheric pressure, or at $25^{\circ}$ and $18 \mathrm{~mm}$. pressure over phosphorus pentoxide. About 5 to 6 hours were required in the former case, and about 16 to 18 hours in the latter. The color of the samples dried at $110^{\circ}$ became tan instead of white.

Anal. Calcd. for $\mathrm{C}_{6} \mathrm{H}_{5} \mathrm{O}_{4} \mathrm{Na} .4 \mathrm{H} 2 \mathrm{O}$ (Mol. wt. 236): Loss of weight, 30.51 ; $\mathrm{Na}, 9.75$. Found: Loss of weight, $30.31,30.22$ (at $110^{\circ}$ ); 29.74 (over $\mathrm{P}_{2} \mathrm{O}_{5}$ at $25^{\circ}$ and a pressure of $18 \mathrm{~mm}$.); $\mathrm{Na}, 10.3$.

(c) Preparation of 5-Hydroxy-2-(methoxymethyl)- $\gamma$-pyrone and Its Aluminum Salt

Dimethyl sulfate, $47.00 \mathrm{~g} \cdot(0.373 \mathrm{~mole})$, was slowly added at room temperature to a stirred solution of $15.00 \mathrm{~g} \cdot(0.106$ mole) of kojic acid and $37.50 \mathrm{~g} \cdot(0.6 \mathrm{~mole})$ of potassium hydroxide (purity 90\%) in $315 \mathrm{ml}$. of water. The reaction mixture warmed up slowly to $37^{\circ}$, but was then cooled to $25^{\circ}$, and subsequently kept below $30^{\circ}$. The initial yellow color changed gradually to red. The addition was completed in 3 hours, whereupon the volume of the solution was reduced to $250 \mathrm{ml}$. at $45-50^{\circ}$ and $20 \mathrm{~mm}$. pressure. While still alkaline, the liquor was extracted continuously for 48 hours with benzene, but the yellow extract left only traces of an oily residue on evaporation. The aqueous residue was then acidified to $\mathrm{pH} 2$ with $50 \%$ sulfuric acid, and was re-extracted continuously with benzene for 21 hours. This dark red extract was treated with 
activated carbon, concentrated to a small volume, and diluted with petroleum ether. A red oil separated, which solidified on chilling, to yield $6.85 \mathrm{~g}$. of an ochre substance melting at $55.5-66.5^{\circ}$. Crystallization from ethyl acetate yielded $1.85 \mathrm{~g} \cdot(11.2 \%)$ of pale tan crystals melting at $75-76^{\circ}$. Recrystallization from toluene - hexane did not raise this melting point, but changed the color of the crystals to white. This substance gave a wine color with aqueous ferric chloride, and had the composition of a monomethyl ether of kojic acid.

The remainder of the product was atill highly colored, and neither treatment with activated carbon nor further crystallizations from ethyl acetate or benzene could improve its purity.

A concentrated benzene solution of this crude fraction was then applied to a short column of alumina, and eluted with benzene. Evaporation of the effluent fractions yielded $0.59 \mathrm{~g}$. of orange-red crystals melting at $252-254^{\circ}$ with decomposition, and $0.34 \mathrm{~g}$. of yellow crystals melting at $265-267^{\circ}$, also with decomposition. These two fractions differed only in their degree of purity, and by crystallization from ethanol they both yielded pale yellow crystals melting at $269-270^{\circ}$ with decomposition. The total weight of the purified substance wes $0.71 \mathrm{~g} \cdot(4 \%)$ Repeated crystallizations from ethanol raised the melting point to $270-$ $271^{\circ}$ with decomposition. This substance also gare a wine color with aqueous ferric chloride, and left a small amount of ash when ignited. A qualitative test demonstrated the presence of aluminum in the ash.

Anal. Caled. for $\mathrm{C}_{6} \mathrm{H}_{5} \mathrm{O}_{3}\left(\mathrm{OCH}_{3}\right)$ (Mol. wt. 156): 0, 53.85; H, 5.13; $\mathrm{OCH}_{3}, 19.9$. Found, $\mathrm{C}, 53.71,53.84 ; \mathrm{H}, 5.25,5.24$; $\mathrm{OCH}_{3}$, $20.4,20.4$. 
Calcd. for $\mathrm{Al}\left[\mathrm{C}_{6} \mathrm{H}_{4} \mathrm{O}_{3}\left(\mathrm{OCH}_{3}\right)\right]_{3}$ (Mol. wt. 492): Ash, 10.36; Al, 5.49; $\mathrm{OCH}_{3}, 18.9$. Found, Ash, $10.43 ; \mathrm{Al}, 5.52 ; \mathrm{OCH}_{3}, 18.6$.

(d) Recovery of 5-Hydroxy-2-(methoxymethyl)-8-pyrone from Its Aluminum Salt

A solution of $0.15 \mathrm{~g} .(0.000305 \mathrm{~mole})$ of the above aluminum salt in $15 \mathrm{ml}$. of water was acidified to ca. $\mathrm{pH} 2$ with a few drops of $50 \%$ sulfuric acid; and was evaporated to an oily residue at $45-50^{\circ}$ and $20 \mathrm{~mm}$. pressure. The residue was dried in vacuo and then boiled for a few minutes with $15 \mathrm{ml}$. of toluene. This extract was decanted from the insoluble residual oil, concentrated on the steambath to a small volume, and diluted with hexane. A crop of white crystals was thus obtained, woighing $0.09 \mathrm{~g}$. and melting at $74.5-76^{\circ}$. A mixture of this material with an authentic sample of 5-hydroxy-2-(methoxymethyl)- $\gamma$-pyrone showed no depression of the melting point.

\section{(e) Methylation of 5-Hydroxy-2-(methoxymethyl)- $\gamma$-pyrone with Diazomethane}

A solution of approximately $0.56 \mathrm{~g} \cdot(0.0133$ mole) of diazomethane in $20 \mathrm{ml}$. of benzene was prepared according to the standard procedure (152), and dried for 2 hours over potassium hydroxide pellets. Finely powdered 5-hydroxy-2-(methoxymethyl $)-\gamma$-pyrone, $0.50 \mathrm{~g} \cdot(0.0032$ mole), was added to the yellow benzene solution in several portions. The solid dissolved readily, with brisk evolution of gas. The golden colored solution was left standing at room temperature for 12 hours, and was then concentrated on the steambath to $2 \mathrm{ml}$., diluted with $2 \mathrm{ml}$. of petroleum ether, and 
shaken while it cooled. A crop of pale yellow crystals formed suddenly in the liquor. These crystals, when recovered on a filter, weighed $0.31 \mathrm{~g}$. and melted at $84-86^{\circ}$. A second, smaller crop of crystals, weighing $0.05 \mathrm{~g}$. and melting at $87.5-89.5^{\circ}$, was obtained by diluting the concentrated filtrate with isopropyl ether, and recrystallizing the crude precipitate from benzene - hexane.

The larger and less pure fraction of crystals was recrystallized from benzene - petroleum ether, giving $0.28 \mathrm{~g}$. (total yield, 60.5\%) of white needles melting at $88.5-89.5^{\circ}$. A final recrystallization from ethanol - isopropyl ether raised the melting point to $89-90^{\circ}$. A mixture of this substance with an authentic sample of 5-methoxy-2-(methoxymethyl)$-\gamma$-pyrone (m.p. $\left.89.5-90^{\circ}\right)$ showed no depression of the melting point.

\section{(f) Benzoylation of Kojic Acid in Aqueous Sodium Hydroxide I}

A solution of $3.00 \mathrm{~g} \cdot(0.0211 \mathrm{~mole})$ of $\mathrm{kojic}$ acid and of $8.46 \mathrm{~g}$. $(0.211$ mole) of sodium hydroxide in $171 \mathrm{ml}$. of water was prepared and stored at room temperature for two days. Then $14.85 \mathrm{~g} \cdot(0.106$ mole) of benzoyl chloride was added slowly, with constant stirring, to this solution over the course of 1.5 hours. Small white globules began to separate immediately from the yellow solution and to combine to larger lumps. Stirring of the mixture was continued for another 50 minutes after the addition of benzoyl chloride was completed. The clear, yellow, alkaline solution was then filtered from the crude solid product, which was dissolved in hot benzene. A total of $2.92 \mathrm{~g}$. of white crystals was recovered from the benzene solution by concentration and by the addition of 
petroleum ether. This material, which melted at $112-123^{\circ}$, could be separated into two components by fractional crystallization from benzene and benzene - petroleum ether. The less soluble fraction, consisting of $0.74 \mathrm{~g}$. of white crystals melting at $128.5-131.5^{\circ}$, was recryatallized from acetone - water, yielding $0.62 \mathrm{~g} \cdot(8.4 \%)$ of white crystals melting at $134-135^{\circ}$. A mixture of this substance with an authentic sample of dibenzoylkojic acid showed no depression of the melting point. The more soluble fraction consisted of $1.41 \mathrm{~g} .(14 \%)$ of soft, matted, very fine white needles, melting at $119.5-121^{\circ}$. Repeated crystallizations from benzene - petroleum ether and from toluene narrowed the melting range to $120-121^{\circ}$. Mixtures of this substance with separate samples of dibenzoylkojic acid and benzoic acid showed depressions of the melting point. The infra-red spectrum of the substance of m.p. 120-121 ${ }^{\circ}$ was identical with that of an equimolecular mixture of dibenzoylkojic acid and benzoic acid. Both spectra were observed on benzene solutions of the compounds (Fig. 2).

Anal. Calcd. for $\mathrm{C}_{2} \mathrm{OH}_{14} \mathrm{O} 6 \mathrm{C}_{7} \mathrm{H}_{6} \mathrm{O}_{2}$ ( $\mathrm{Mol}$. wt. 472): 0, 68.64; H, 4.24 . Found: C, $68.82,68.98 ; \mathrm{H}, 4.36,4.35$.

(g) Benzoylation of Kojlc Acid in Aqueous Sodium Hydroxide II

A solution of $5.00 \mathrm{~g} \cdot(0.0352$ mole $)$ of kojic acid and $7.30 \mathrm{~g}$. $(0.177$ mole) of sodium hydroxide (purity $97 \%$ ) in $177 \mathrm{ml}$. of water was stored at room temperature for 2 hours. Then $19.75 \mathrm{~g} \cdot(0.141$ mole) of benzoyl chloride was added slowly from a separatory funnel to the stirred solution at room temperature, over a period of 2.75 hours. A granular 
solid separated from the solution, and was recovered by filtration. A solution of $5.80 \mathrm{~g} .(0.141 \mathrm{~mole})$ of sodium hydroxide in $50 \mathrm{ml}$. of water was added to the acidic filtrate, and another $19.75 \mathrm{~g} \cdot(0.141$ mole) of benzoyl chloride was added to the stirred mixture, drop by drop, over a period of 2.5 hours. Stirring was contimued for one additional hour, then the sticky, solid reaction product was recovered on a filter. The filtrate was again acid to litmus. The two portions of crude solid were dried, combined, and subjected to fractional crystallization. The solvents used for this purpose were dry benzene and dry hexane. The crude product proved to be a mixture of two derivatives of kojic acid with benzoic acid. The lest was the most soluble component of the mixture, and was thus readily removed. Of the remainder, the more soluble fraction consisted of $5.88 \mathrm{~g} .(35.5 \%)$ of matted white needles melting at $117-118^{\circ}$. A mixture of this fraction with a sample of the substance of m.p: 120-121 obtained from the benzoylation of kojic acid according to Experiment I ( $f$ ), showed no depression of the melting point. The less soluble fraction consisted of $1.55 \mathrm{~g}$. (18\%) of white crystals melting at $142-143^{\circ}$. Mixtures of this substance with samples of 5-benzoxy-2-(hydroxymethyl)- $\gamma$-pyrone, prepared from anhydrous sodium kojate (1) and from 5-benzoxy-2-(triphenylmethoxymethyl)- $r$-pyrone (k), showed no depression of the melting point.

Anal. Calcd. for $\mathrm{C}_{1} \mathrm{H}_{10} \mathrm{O}_{5}$ (Mol. wt. 246): C, 63.42; H, 4.07. Found: c, 63.25, 63.30; $\mathrm{H}, 4.12,4.35$.

\section{(h) Benzoylation of Kojic Acid in Dry Pyridine I}

To a chilled solution of $2.00 \mathrm{~g} \cdot(0.0141 \mathrm{~mole})$ of kojic acid in 
$20 \mathrm{ml}$. of dry pyridine was added $5.00 \mathrm{~g} \cdot(0.0356 \mathrm{~mole})$ of benzoyl chloride in several portions. The solution was stored overnight at room temperature, then the solvent was evaporated in a current of dry air, with gentle heating, to leave a moist pulp. In order to separate the products from pyridine hydrochloride, the residue was treated with hot benzene, in which most dissolved. The product was regained from the filtered benzene solution in several fractions. The less soluble portion consisted of $3.69 \mathrm{~g}$. of a white, crystalline solid melting at $124-132^{\circ}$, and the more soluble portion consisted of $1.00 \mathrm{~g}$. of soft, matted white needles melting at $116-118^{\circ}$. The former material was purified by recrystallization from benzene - petroleum ether and from acetone - water, yielding $3.04 \mathrm{~g}$. of pure product melting at $134-135^{\circ}$, and a smaller portion of the same product, $0.41 \mathrm{~g}$., melting at $133-134^{\circ}$ (total yield, 70\%). A mixture of this material with dibenzoylkojic acid showed no depression of the melting point. The more soluble portion of the crude product was recrystallized from benzene and from benzene - petroleum ether to yield $0.66 \mathrm{~g} .(10 \%)$ of matted white needles melting at $119.5-120.5^{\circ}$. A mixture of this substance with a sample of the addition compound of dibenzoylkojic acid and benzoic acid (m.p. 120-121 ${ }^{\circ}$ ), obtained from the benzoylation of kojic acid in aqueous sodium hydroxide $(f, g)$ showed no depression of the melting point.

\section{(i) Benzoylation of Kojic Acid in Dry Pyridine II}

A solution of $15.00 \mathrm{~g} \cdot(0.1055$ mole) of kojic acid in $140 \mathrm{ml}$. of dry pyridine was chilled to $5^{\circ}$. To this solution was added, in several 
portions, $37.50 \mathrm{~g} \cdot(0.268 \mathrm{~mole})$ of benzoyl chloride, the mixture being chilled and shaken between additions. A yellow sludge formed which was stored at room temperature overnight, then poured into $1300 \mathrm{ml}$. of chilled $5 \%$ aqueous sulfuric acid. The oil which separated solidified completely after a few minutes of stirring, and the crude solid was recovered on a filter and partly dried by suction. This product was crystallized from acetone - water, to yield $36.40 \mathrm{~g} \cdot(98.5 \%)$ of white crystals melting at $134-135^{\circ}$, undepressed by admixture with an authentic sample of dibenzoylkojic acid.

\section{(i) Preparation of 5-Benzoxy-2-(triphenylmethoxymethyl)- $\gamma$-pyrone}

This substance was prepared according to Yabuta's (72) method; but the following details are recorded, because the original source of information (Chemical Abstracts) did not deal with them. Treatment of $5.00 \mathrm{~g} \cdot(0.0352 \mathrm{~mole})$ of kojic acid with $11.00 \mathrm{~g} \cdot(0.0396 \mathrm{mol \theta})$ of triphenylmethyl (trityl) chloride in $50 \mathrm{ml}$. of dry pyridino gave a brown paste. This crude product, when crystallized from acetone - water, yielded $11.69 \mathrm{~g}$. of tan crystals melting at $130-166^{\circ}$. Purification of this mixture proved to be laborious, and it was found more convenient to boil the crude solid with ether, in which tritylkojic acid was almost insoluble. The residual $6.08 \mathrm{~g}$. of ether-insoluble crystals, melting at

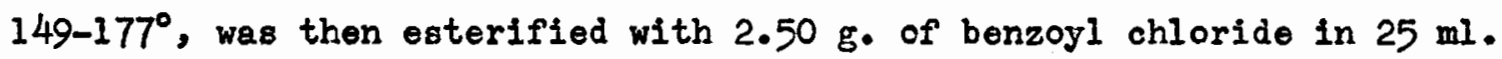
of dry pyridine at room temperature. This reaction mixture yielded $7.87 \mathrm{~g}$. of a white solid melting at $134-164^{\circ}$. Crystallization of this material from acetone - ethanol gave $3.03 \mathrm{~g}$. of white crystals melting 
above $200^{\circ}$. To obtain pure 5-benzoxy-2-(triphenylmethoxymethyl)- $\gamma$-pyrone, these crystals were recrystallized twice from dioxane - water, which was found to be the most satisfactory solvent for this purpose. Yield, $2.22 \mathrm{~g}$. (13\%) melting at $212.5-214^{\circ}$. Yabuta (72) reported a melting point of 206$208^{\circ}$ for this compound.

\section{(k) Detritylation of 5-Benzoxy-2-(triphenylmethoxymethyl)- $\gamma$-pyrone}

A solution of $0.36 \mathrm{~g} \cdot(0.000737$ mole $)$ of 5-benzoxy-2-(triphenylmethoxymethyl)- $\gamma$-pyrone in $15 \mathrm{ml}$. of hot $80 \%$ aqueous acetic acid was heated under reflux for 35 minutes. The stepwise addition of water to the solution, and cooling, caused the deposition of two crops of a white solid, totalling $0.19 \mathrm{~g}$. and melting at $159-160.5^{\circ}$ (triphenyl carbinol). The filtrate was evaporated at $50^{\circ}$ and $10 \mathrm{~mm}$. pressure to leave a colid residue, which was purified from benzene, yielding $0.10 \mathrm{~g} \cdot(55 \%)$ of white crystals molting at $139.5-141^{\circ}$. Further crystallizations from benzene raised the melting point to $142-143^{\circ}$. This sample of 5 -benzoxy-2-(hydroxymethyl)- $\gamma$-pyrone was Identical, as shown by mixed melting point determinations, with those obtained from the reaction between anhydrous sodium kojate and benzoyl chloride in dry benzene ( 1 ), and as a by-product in the benzoylation of kojic acid in aqueous alkali $(\mathrm{g})$. Yabuta reported a melting point of $135-136^{\circ}$ for 5-benzoxy-2-(hydroxymethyl) - $\gamma$-pyrone prepared by detritylation of 5-benzoxy-2-(triphenylmethoxymethyl)- $\gamma$-pyrone (72), and of $135^{\circ}$ for the same compound prepared by the treatment of kojic acid with benzoyl chloride in other (4). 
(1) Preperation of 5-Benzoxy-2-(hydroxymethyl)- $\gamma$-pyrone from Anhydrous Sodium Rofate

Preparation of Anhydrous Sodium Kojate:- A suspension of $2.00 \mathrm{~g}$. $(0.0141$ mole $)$ of kojic acid in $10 \mathrm{ml}$. of water was placed in a $100 \mathrm{ml}$. round-bottom flask with a standard taper ground-glass neck. Six drops of a phenolphthalein solution were added to this suspension, and then enough $5 \%$ aqueous sodium hydroxide to produce definite pink color. All of the kojic acid dissolved in this process. The solution was evaporated completely at $45-50^{\circ}$ and a pressure of $20 \mathrm{~mm} .$, and the residual pale cream yellow solid was dried over phosphorus pentoxide for 20 hours, at $25^{\circ}$ and a pressure of $18 \mathrm{~mm}$.

Benzoylation:- Fifty milliliters of dry benzene and $2.50 \mathrm{~g} \cdot(0.0178$ mole) of benzoyl chloride were added to the anhydrous sodium kojete, still in the original flask. The tightly stoppered flask was then mechanically shaken at room temperature for 26 hours, the crust of solid adhering to the walls of the flask being loosened after the first 2 to 3 hours of shaking by tapping the flask on the bench top. When the shaking was stopped, $30 \mathrm{ml}$. of benzene was added to the white sludge in the flask, the contents were heated to boiling, and the hot benzene solution filtered. The residual solid was extracted with a further volume of boiling benzene, and then with $30 \mathrm{ml}$. of water to dissolve most of the sodium chloride. The residue, now largely organic, was recovered by filtration and boiled with $100 \mathrm{ml}$. of benzene. After filtration from traces of insoluble material, this benzene solution was combined with the two benzene extracts, and allowed to cool to room temperature. A crop of long 
soft white needles formed, which was recovered on a filter. Yield, $2.53 \mathrm{~g}$. melting at $143 \cdot 5-144.5^{\circ}$. Recrystallization from benzene and from acetone - water did not raise the melting point. A small second crop of needles was obtained by concentrating the filtrate on the steam bath to $10 \mathrm{ml}$. and adding $15 \mathrm{ml}$. of petroloum ether. After recryatallization from acetone - water this fraction weighed $0.22 \mathrm{~g}$. (total yield, $79.5 \%$ ) and melted at $143-144^{\circ}$. Mixtures of this substance with separate samples of 5-benzoxy-2-(hydroxymethyl)- $\gamma$-pyrone, obtained from the detritylation of 5-benzoxy-2-(triphenylmethoxymethyl)- $\gamma$-pyrone $(k)$ and as a by-product in the benzoylation of kojic acid in aqueous alkali $(g)$, showed no depression of the melting point. The melting points recorded in the literature $(4,72)$ for this substance were $8-9^{\circ}$ lower $[$ see $(k)]$.

(m) Resolution of the Molecular Addition Compound of Dibenzoylkojic Acid and Benzoic Acid

A solution of $0.50 \mathrm{~g} \cdot(0.00106 \mathrm{~mole})$ of the addition compound (m.p. $\left.120-121^{\circ}\right)$ in $5 \mathrm{ml}$. of warm acetone was diluted with an equal volume of cold water. A white crystalline precipitate formed immediately; the mixture was cooled to room temperature, and the solid recovered on a filter. Yield, $0.37 \mathrm{~g}$. (100\%); melting point, 134-135 . A mixture of this substance with dibenzoylkojic acid showed no depression of the melting point. The clear filtrate, when concentrated on the steam bath to half of its original volume and then cooled, y1elded $0.12 \mathrm{~g}$. (92.5\% of one equivalent wt.) of white crystals which melted at $116.5-119^{\circ}$. After crystallization from hot water this substance melted at $121.5-122^{\circ}$. 
A mixture with an authentic sample of benzoic acid showed no depression of the melting point.

Dibenzoylkojic acid crystallized also from the solutions of the addition compound in dioxane (on dilution with water), $95 \%$ ethanol, ethyl acetate, absolute methanol and pyridine (on cooling). When a solution of the addition compound in benzene was extracted with $5 \%$ aqueous sodium carbonate, only dibenzoylkojlc acid was regained by evaporation of the benzene solution.

The adition compound dissolved readily with a brisk erolution of gas when added to a solution of diazomethane in benzene. Crystals of dibenzoylkojic acid were recovered the following day by working up the yellow benzene solution, which had the characteristic odor of methyl benzoate.

When the hydrogenation of the addition compound was attempted in glacial acetic acid over a platinum catalyst, at room temperature and atmospheric pressure, only a sirup and benzoic acid were recovered. The sirup was not investigated further. A reduction, heating with zinc in glacial acetic acid on the steam bath, yielded products which were identical with those obtained from dibenzoylkojic acid in the same reaction, namely benzoic acid and benzoylallomaltol ( $v$ ).

A treatment of the addition compound with hydroxylamine hydrochloride in pyridine for 12 hours at room temperature, produced a $51 \%$ yield of 2-(benzoxymethyl)-5-hydroxy- $\gamma$-pyrone, a substance obtained in good yield from dibenzoylkojic acid under identical conditions ( $n$ ). The some substance was formed when an excess of benzoylhydrazine was added to a glacial acetic acid solution of the addition compound at room temperature. 
A second product of this reaction was $N, N^{\prime}$-dibenzoylhydrazine, melting at $240.5-241^{\circ}$, which was identified by a mixed melting point determination with a sample (m.p. $241^{\circ}$ ) synthesized from benzoylhydrazine and benzoyl chloride in dry pyridine at room temperature. The melting point of $\mathrm{N}, \mathrm{N}^{\prime}$-dibenzoylhydrazine is given as $241^{\circ}$ in the literature (153).

The addition compound was, however, regained substantially without change from its solutions in benzene, hexane - benzene, toluene, and glacial acetic acid, as well as after an attempted hydrogenation in glacial acetic acid over $5 \%$ palladized charcoal, at room temperature and atmospheric pressure.

(n) Preferential Dobenzoylation of Dibenzoylkojic Acid at Position 5 by Hydroxylamine Hydrochloride in the Presence of Weak Bases

A solution of $0.30 \mathrm{~g} \cdot(0.000857 \mathrm{~mole})$ of dibenzoylkojic acid and $0.12 \mathrm{~g} \cdot(0.00173$ mole $)$ of hydroxylamine hydrochloride in $4 \mathrm{ml}$. of pyridine was left standing at room temperature for 12 hours, then diluted with $20 \mathrm{ml}$, of cold water. A white crystalline precipitate formed, which was recovered, after a few hours of chilling, by filtration. Yield, $0.19 \mathrm{~g} .(90 \%)$, melting at $178.5-179.5^{\circ}$. After crystallization from ethanol - water the white needles melted at 180-181 . This substance gave a wine color with an alcoholic solution of ferric chloride.

The preferential hydrolysis of dibenzoylkojic acid at position 5 could also be achieved by heating for 30 minutes with 3 equivalents of hydroxylamine hydrochloride and 3 equivalents of pyridine in $95 \%$ ethanol (yield $83 \%$ ); or by heating a suspension of dibenzoylkojic acid and 
3 equivalents each of hydroxylamine hydrochloride and sodium acetate in $80 \%$ ethanol on the steam bath until all the solid had completely dissolved. In this latter case, the pure product crystallized on cooling the solution to room temperature (yield $74 \%$ ).

Three equivalents of either sodium acetate or hydroxylamine hydrochloride alone had no appreciable effect on dibenzoylkojic acid in boiling ethanol.

Anal. Calcd. for $\mathrm{C}_{6} \mathrm{H}_{5} \mathrm{O}_{4}\left(\mathrm{COC6H}_{5}\right)$ ( $\mathrm{Mol}$. wt. 246): C, 63.42; $\mathrm{H}, 4.07$; Coc6 $\mathrm{H}_{5}, 42.7$. Found: $0,63.54,63.59 ; \mathrm{H}, 4.08,4.23 ; \mathrm{COOC}_{6} \mathrm{H}_{5}$, $41.7,41.0$.

\section{(0) Benzoylation of 2-(Benzoxymethyl)-5-hydroxy- $\gamma$-pyrone in Dry Pyridine}

Benzoyl chloride, $0.20 \mathrm{~g} \cdot(0.00143 \mathrm{~mole})$, was added in two portions to an ice-cold solution of $0.30 \mathrm{~g} \cdot(0.00122 \mathrm{~mole})$ of 2 -(benzoxymethyl)5-hydroxy- $\gamma$-pyrone in $3 \mathrm{ml}$. of dry pyridine. The mixture was allowed to stand at room temperature for 7 hours before being poured into a large volume of ice-cold $10 \%$ sulfuric acid and stirred until the separation of a white precipitate was completed. The crude solid product weighed $0.46 \mathrm{~g}$. and melted at $126-129.5^{\circ}$. Two crystallizations from benzene - petroleum ether yielded $0.38 \mathrm{~g} .(89 \%)$ of white crystals melting at $131-133^{\circ}$. Recrystallization from acetone - water yielded $0.30 \mathrm{~g}$. of white crystals melting at $134-135^{\circ}$. The identity of this substance with dibenzoylkojic acid was established by a mixed melting point determination, which showed no depression. 
(p) Methylation of 2-(Benzoxymethyl)-5-hydroxy- $\gamma$-pyrone with Dizzomethane

One gram $(0.00406$ mole) of finely powdered 2-(benzoxymethyl)-5-hydroxy- $\gamma$-pyrone was added, in amall portions, over a period of 10 minutes to a solution of approximately $0.5 \mathrm{~g} \cdot(0.0119 \mathrm{~mole})$ of diazomethane in $18 \mathrm{ml}$. of benzene. The additions caused a brisk evolution of gas. The mixture was allowed to stand at room temperature for 6 hours, during which period practically all of the solid dissolved. The golden yellow solution was concentrated on the steam bath to $4-5 \mathrm{ml}$. Cooling of the concentrated solution caused the deposition of crystals, which were recovered by filtration and recrystallized from benzene - potroleum ether. The yield was $0.52 \mathrm{~g}$. (49\%) of off-white crystals, melting at $109-110^{\circ}$. Further recrystallizations from benzene - petroleum ether raised the melting point to $110-111^{\circ}$. Treatment of this product with activated carbon in ethanol changed its color to snow-white, but recrystallization from othanol - water left the melting point unchanged.

Anal. Calcd. for $\mathrm{C}_{1} 3 \mathrm{HgO4}\left(\mathrm{OCH}_{3}\right)(\mathrm{Mol}$. wt. 260): C, 64.62; H, 4.62; $\mathrm{OCH}_{3}$, 11.9. Found: $\mathrm{C}, 64.71,64.92 ; \mathrm{H}, 4.55,4.75$; $\mathrm{OCH}_{3}$, $12.0,12.1$.

(a) Benzoylation of 2-(Hydroxymethyl)-5-methoxy- $\gamma$-pyrone in Dry Pyridine

A solution of $1.00 \mathrm{~g} \cdot(0.00641$ mole) of 2-(hydroxymethyl)-5-methoxy- $\gamma$-pyrone, obtained by the methylation of kojic acid with one equivalent of dimethylsulfate in aqueous potassium hydroxide (75), was made up in $15 \mathrm{ml}$. of dry pyridine and chilled to $0^{\circ}$. Benzoyl chloride, 1.10 g. ( 0.00785 mole), was added in several portions. The solution was 
left standing at room temperature for 7 hours in a tightly stoppered flask, and was then worked up according to the procedure used in the benzoylation of 2-(benzoxymethyl)-5-hydroxy- $\gamma$-pyrone (0). The crude product, a solid melting at $104-109^{\circ}$, weighed $1.35 \mathrm{~g}$. Recrystallization from ethanol - water and benzene - petroleum ether yielded $1.11 \mathrm{~g} .(67 \%)$ of white crystals melting at $110-111^{\circ}$. A mixture of this substance with a sample of 2-(benzoxymethyl)-5-methoxy- $\gamma$-pyrone (m.p. 110-111\%), obtained by the methylation of 2 -(benzoxymethyl)-5-hydroxy- $\gamma$-pyrone $(p)$, did not show a depression of the melting point.

\section{(r) Preparation of 2-(Acetoxymethy 1$)-5$-benzoxy- $\gamma$-pyrone}

A solution of $2.80 \mathrm{~g} \cdot(0.0114 \mathrm{~mole})$ of 5-benzoxy-2-(hydroxymethyl)$-\gamma$-pyrone in $24 \mathrm{ml}$. of dry pyridine was chilled and then mixed with $4.10 \mathrm{~g} \cdot(0.0402 \mathrm{~mole})$ of acetic anhydride. The resulting clear solution was allowed to stand overnight at room temperature. Then the solution was poured with atirring into $200 \mathrm{ml}$. of cold water, and the off-white crystalline solid which separated was recovered by filtration, dried, and weighed. Yield: $3.06 \mathrm{~g} .(93 \%)$ melting at $142.5-143.5^{\circ}$. Recrystallization from benzene (twice) and acetone - water raised the melting point only slightly. The final pure sample consisted of white plates melting

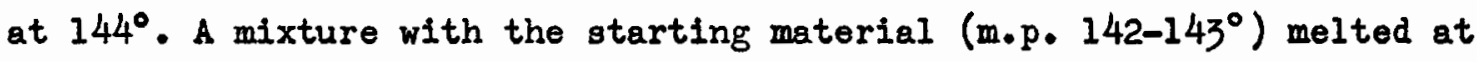
$120-127^{\circ}$.

Anal. Calcd. for $\mathrm{C}_{1} 5_{\mathrm{H}} 12 \mathrm{O} 6$ (Mol. wt. 288): C, 62.50; H, 4.18. Found: C, $62.82,62.48 ; \mathrm{H}, 4.33,4.49$. 
(s) Debenzoylation of 2-(Acetoxymethyl)-5-benzoxy- $\gamma$-pyrone

A solution of $0.96 \mathrm{~g}$. $(0.00334 \mathrm{~mole})$ of 2-(acetoxymethyl)-5-benzoxy$\gamma$-pyrone and $0.46 \mathrm{~g} .(0.00663$ mole $)$ of hydroxylamine hydrochloride in $13 \mathrm{ml}$. of pyridine was allowed to stand for 12 hours at room temperature. Then the yellow solution was diluted with 6 volumes of cold water, and the resulting clear liquid was extracted with 5 lots of benzene, totalling $60 \mathrm{ml}$. The aqueous residue was evaporated to dryness at $50^{\circ}$ and a pressure of $10 \mathrm{~mm}$, the yellow solid residue was extracted with $30 \mathrm{ml}$. of boiling benzene, and the benzene solution separated from a benzeneinsoluble oil by decantation. The two benzene extracts were dried over anhydrous sodium sulfate, evaporated on the steam bath, and their solid residues combined and recrystallized from a small volume of ethanol. Yield of white needles: $0.47 \mathrm{~g} \cdot$ of $\mathrm{m} \cdot \mathrm{p} \cdot 134-135^{\circ}$ and $0.04 \mathrm{~g} \cdot$ of m.p. $130-132^{\circ}$, a total of $0.51 \mathrm{~g} \cdot(83 \%)$ of 2 -(acetoxymethyl)-5-hydroxy- $\gamma-$ pyrone. This substance gave a wine color with aqueous ferric chloride, and when acetylated in the presence of sodium acetate (71) yielded diacetylkojic acid, m.p. 101-102 . The identity of this latter derivative was established by a mixed melting point determination with an authentic sample (m.p. 101-102 $)$. The melting point of 2-(acetoxymethyl)5 -hydroxy- $\gamma$-pyrone is recorded in the literature es $133.5^{\circ}$ (71) and as $136^{\circ}(54)$

(t) Debenzoylation of 5-Benzoxy-2-(triphenylmethoxymethyl)- $\gamma$-pyrone

A solution of $0.29 \mathrm{~g} \cdot(0.000595$ mole) of 5-benzoxy-2-(triphenylmethoxymethyl $)-\gamma$-pyrone $(j)$ and of $0.35 \mathrm{~g} \cdot(0.00504 \mathrm{~mole})$ of 
hydroxylamine hydrochloride in $7 \mathrm{ml}$. of pyridine was allowed to atand overnight at room temperature. Then the clear, yellow solution was diluted with about $45 \mathrm{ml}$. of cold water, and the precipitate of white crystals was recovered after a few hours by filtration, washod with water and dried. Yield: $0.22 \mathrm{~g}$. melting at $171-176^{\circ}$. Recrystallization of this substance from benzene - petroleum ether, dioxane - water, and finally carbon tetrachloride - isopropyl ether (the last solvent pair was the most satisfactory) yielded $0.15 \mathrm{~g} \cdot(66 \%)$ of practically pure tritylkojic acid as white crystals melting at $180-181.5^{\circ}$. This substance gave a wine color with ferric chloride in alcohol, and when mixed with a sample of tritylkojic acid (m.p. $181-182^{\circ}$ ), obtained from the tritylation of kojic acid in dry pyridine ( 72 ), no depression of the melting point was observed. Yabute (72) gave the melting point of tritylkojic acid as 185$185.5^{\circ}$

\section{(u) Catalytic Hydrogenation of Dibenzoylkojic Acid}

A suspension of the catalyst was prepared by stirring $0.03 \mathrm{~g}$. of platinum oxide in $25 \mathrm{ml}$. of glacial acetic acid under an atmosphore of hydrogen, at $25^{\circ}$ and at a pressure of $755 \mathrm{~mm}$, until the uptake of hydrogen ceased. An all-glasa apparatus and a magnetic stirrer wero used. To the suspension of the catalyst was added $0.50 \mathrm{~g} \cdot(0.00143$ mole) of dibenzoylkojic acid, which dissolved in the acetic acid after a few minutes of stirring. The mixture was stirred under hydrogen at the same temperature and pressure as noted initially, and the uptake of the gas slowed down gradually, but had not yet stopped completely when the 
hydrogenation was interrupted after 4.75 hours. At this time the absorption was $131 \mathrm{ml}$. of hydrogen, corresponding to 7.4 atoms per molecule of dibenzoylkojic acid. The acetic acid solution was filtered from the catalyst, and evaporated on the steam bath to an oil which became semisolid when kept over anhydrous calcium chloride in a vacuum desiccator. This residue was dissolved in benzene, and the benzene solution was extracted with $5 \%$ aqueous sodium carbonate. Acidification of the aqueous extract caused the separation of $0.10 \mathrm{~g}$. (28.6\% of 2 equivalent wts.) of a white solid melting at $118-120^{\circ}$. Recrystallization from water raised the melting point to $122.5^{\circ}$, and a mixture of this substance with an authentic sample of benzoic acid showed no depression of the melting point. The benzene solution was dried over anhydrous sodium sulfate and then evaporated completely on the steam bath. The residual sirup did not change in state after drying over calcium chloride at reduced pressure, and could not be crystallized, and was not investigated further.

\section{(v) Preparation of Benzoylallomaltol by the Reductive Fission of} Dibenzoylkojic Acid

Ninety grams of technical grade zinc dust was added to a hot solution of $15.00 \mathrm{~g} \cdot(0.0428 \mathrm{~mole})$ of dibenzoylkojic acid in $300 \mathrm{ml}$. of glacial acetic acid; the mixture was protected from moisture by a calcium chloride drying tube and was heated on the steam bath for 2.5 hours, with occasional shaking. Then the hot acetic acid solution was filtered, the residual zinc rinsed with some more hot glacial acetic acid, and 
the combined solutions were evaporated completely at $60^{\circ}$ and a pressure of $20 \mathrm{~mm}$. The brown oily residue became semi-solid when stored over potassium hydroxide in vacuo. Extraction of this residue with $370 \mathrm{ml}$. of boiling isopropyl ether, followed by filtration, yielded a yellow solution and $2.76 \mathrm{~g}$. of a mixture of zinc salts. On cooling to room temperature, the extract deposited a crop of pale tan prismatic needles, which were recovered on a filter after 12 hours. Yield, $2.76 \mathrm{~g}$. melting at $123-125^{\circ}$. The filtrate was extracted with $10 \%$ aqueous sodium carbonate. Acidification of the aqueous extract yielded $4.08 \mathrm{~g}$. of a white solid melting at $121 \cdot 5-122 \cdot 5^{\circ}$. A mixture with an authentic sample of benzoic acid showed no depression of the melting point. A further $0.65 \mathrm{~g}$. of benzoic acid, m.p. 121-122 $2^{\circ}$ was obtained by the acidification of an aqueous suspension of the zinc salts (total yield, $90.5 \%$ of 1 equivalent wt.). After the extraction with sodium carbonate the residual isopropyl other solution was washed with water, dried over anhydrous sodium sulfate and concentrated on the steam bath to $170 \mathrm{ml}$. to give $0.80 \mathrm{~g}$. of prismatic needles melting at $123.5-125.5^{\circ}$. The two portions of this substance were combined and recrystallized from benzene - isopropyl ether, to give $3.17 \mathrm{~g} .(32 \%)$ of white needles melting at $124.5-126^{\circ}$. Further recrystallizations from the same solvent raised the melting point to $126-127^{\circ}$

Anal. Calcd. for $\mathrm{C}_{13} \mathrm{H}_{10} \mathrm{O}_{4}$ (Mol. wt. 230): C, 67.83; H, 4.35. Found: $C, 67.94 *, 67.78,67.70 ; \mathrm{H}, 4.82 *, 4.81,4.97$.

* Schwarzkopf Microanalytical Laboratory 
(w) Debenzoylation of Benzoylallomaltol

A solution of $2.40 \mathrm{~g} .(0.0104$ mole $)$ of benzoylallomaltol and $0.95 \mathrm{~g}$. $(0.0138$ mole) of hydroxylamine hydrochloride in $25 \mathrm{ml}$. of pyridine was left standing at room temperature for 11 hours. The solution, now yellow, was evaporated completely at $55^{\circ}$ and a pressure of $20 \mathrm{~mm}$, and the residual brown oil was dried over potassium hydroxide at a reduced pressure. Part of the oil crystallized in this process. The heterogeneous residue was bolled with several portions of benzene; the benzene extracts were decanted from an insoluble oil, were combined, concentrated to $5 \mathrm{ml}$, and diluted with $3 \mathrm{ml}$. of hexane. On cooling to room temperature, a crop of white crystals formed and were removed by filtration; weight, $0.97 \mathrm{~g}$. and melting point 146-149 . A second, smaller crop of crystals, weighing $0.08 \mathrm{~g}$. and melting at $143-148^{\circ}$, separated from the filtrate after dilution with isopropyl ether. The two fractions were combined and recrystallized from isopropyl ether - ethyl acetate, to give $0.93 \mathrm{~g} \cdot(71 \%)$ of white crystals melting at $150-151^{\circ}$. This substance was soluble in water, gave a wine color with aqueous ferric chloride, and when added to a solution of diazomethane in benzene, dissolved with the vigorous evolution of gas. A mixed melting point determination with an authentic sample of allomaltol showed no depression. Allomaltol (m.p. 150-151 $1^{\circ}$ was synthesized from 2-(chloromethyl)-5-hydroxy- $\gamma$-pyrone (77) by reduction with zinc in glacial acetic acid $(8,76)$.

Anal. Calcd. for $\mathrm{C}_{6} \mathrm{H}_{6} \mathrm{O}_{3}$ (Mol. wt. 126): C, 57.14; H, 4.76. Found: $0,57.09 *, 57.16,56.98 ; \mathrm{H}, 5.24 *, 5.22,4.93$. 
(x) Benzoylation of Allomaltol in Dry Pyridine

A solution of $0.10 \mathrm{~g} .(0.000792 \mathrm{~mole})$ of allomaltol in $2 \mathrm{ml}$. of dry pyridine was chilled, $0.14 \mathrm{~g} \cdot(0.001$ mole $)$ of benzoyl chloride was added in two portions, the mixture was shaken and again chilled, and then stored at room temperature for 24 hours in a tightly stoppered vial. When the mixture was poured into $20 \mathrm{ml}$. of chilled $5 \%$ aqueous sulfuric acid, a crude solid separated which was recovered by filtration. Recrystallization of the precipitate from isopropyl ether - ethyl acetate gave $0.13 \mathrm{~g} \cdot(56.5 \%)$ of white crystals, melting at $120-126^{\circ}$. This crop of benzoylallomaltol was contaminated with a small amount of benzoic acid, which could easily be removed by recrystallization from isopropyl ether - hexane. The pure product melted at $126.5-127^{\circ}$, and a mixture of this material with a sample of benzoylallomaltol (m.p. 126-127 ), prepared by reductive fission from dibenzoylkojic acid ( $v$ ), did not show any depression of the melting point.

(y) Preparation of Dibenzoylkojic Acid $\alpha$ - Disemicarbazone"

A mixture of $14.00 \mathrm{~g} \cdot(0.04 \mathrm{~mole})$ of dibenzoylkojic acid, $14.00 \mathrm{~g}$. $(0.125$ mole $)$ of semicarbazide hydrochloride, $14 \mathrm{ml} \cdot(0.174$ mole) of pyridine, $40 \mathrm{ml}$. of water and $350 \mathrm{ml}$. of ethanol was heated to boiling. The resulting clear solution was kept under reflux for 50 minutes, then transferred to a suitable flask and allowed to cool to room temperature. A crop of yellow-white crystals, which formed over the course of six days, was recovered by filtration, and was then extracted for 15-20 minutes on the steam bath with $50 \mathrm{ml}$. of ethyl acetate, $100 \mathrm{ml}$. of water 
and $50 \mathrm{ml}$. of dioxane in succession. When the product was recovered from the last of these three solutions by filtration, it was white and had a microcrystalline appearance. Yield: $6.04 \mathrm{~g} \cdot$, melting at $212^{\circ}$ with decomposition. A second, smaller crop of similar crystals was deposited by the yellow reaction liquor over the course of 15 additional days. This crop was purified in the same way, to give 1.92 g. (total yield, 41.5\%) melting also at $212^{\circ}$ with decomposition. This "disemicarbazone" was insoluble in practically all liquids except glacial acetic acid. A sample was purified for analysis by solution in a large volume of glacial acetic acid at $60-70^{\circ}$, and by reprecipitation by adding 4 to 5 rolumes of cold water. The white solid melted at $215^{\circ}$ with decomposition.

Anal. Calcd. for $\mathrm{C}_{22} \mathrm{H}_{22 \mathrm{~N}} \mathrm{O}_{7}$ (Mol. wt. 482): C, 54.78; H, 4.56; N, 17.4 . Found: C, $54.81,54.83 ; \mathrm{H}, 4.73,4.83 ; \mathrm{N}, 16.9$.

\section{(z) Preparation of Dibenzoylkojic Acid B-"Disemicarbazone"}

A mixture of $5.00 \mathrm{~g} \cdot(0.0143 \mathrm{~mole})$ of dibenzoylkojic acid, $3.20 \mathrm{~g}$. $(0.0287$ mole $)$ of semicarbazide hydrochloride, $5 \mathrm{ml} \cdot(0.0621 \mathrm{~mole})$ of pyridine, $10 \mathrm{ml}$. of water and $60 \mathrm{ml}$. of ethanol was heated to boiling, and the resulting clear solution was kept at the temperature of reflux for 50 minutes. The solution, now yellow, when concentrated on the steam bath to about $10 \mathrm{ml}$. doposited a yellow-white solid, weighing $1.75 \mathrm{~g}$. and melting at $179-181^{\circ}$ with decomposition. The filtrate was diluted with water, the oil which separated was caused to solidify by chilling, the supernatant liquid was decanted, and the semi-solid oily material was boiled with ethyl acetate. This treatment left undissolved a small 
crop of white powder which was recovered by filtration; weight, $0.33 \mathrm{~g}$. and m.p. $183-184^{\circ}$ with decomposition. The red ethyl acetate extract was left standing at room temperature overnight, and the crop of white crystals which formed was recovered on a filter, yielding $0.61 \mathrm{~g}$. melting at $120.5-121.5^{\circ}$ with decomposition. The high-melting fractions were combined and extracted on the steam bath in succession with othyl acetate and water, to leave undissolved $1.65 \mathrm{~g}$. (24\%) of a white microcrystalline solid melting at $213^{\circ}$ with decomposition. This substance had all the properties of dibenzoylkojic acid $\alpha$-disemicarbazone". The low-melting fraction was recrystallized from ethyl acetate and from ethanol - water to give $0.40 \mathrm{~g} \cdot(6 \%)$ of white crystals melting at $170^{\circ}$ with decomposition. Further recrystallizations from ethanol - water and from ethanol methenol raised the melting point to $172-172.5^{\circ}$ with decomposition.

Anal. Calcd. for $\mathrm{C}_{22} \mathrm{H}_{22 \mathrm{~N}} 607$ (Mol - wt. 482): C, 54.78; H, 4.56; N, 17.4 . Found: $\mathrm{C}, 54.64,54.54 ; \mathrm{H}, 4.95,4.90 ; \mathrm{N}, 16.6$.

(aa) Preparation of Dibenzoylkojic Acid $\alpha$-"Monosemicarbazone"

A suspension of $3.05 \mathrm{~g} \cdot(0.00633 \mathrm{~mole})$ of dibenzoylkojlc acid $\alpha$ - "disemicarbazone" in $70 \mathrm{ml}$. of glacial acetic acid was stirred at room temperature, while a concentrated aqueous solution of $10.80 \mathrm{~g}$. $(0.157$ mole) of sodium nitrite was added very slowly in three equal portions. Addition periods of 1 to 1.5 hours were spaced 2 hours apart, and otirring was continued for 1 further hour after the addition was completed. The mixture was left standing at room temperature overnight, then the yellow-green solution was filtered from the solid residue. The 
residue was washed free of inorganic salts on the filter, and proved to be $0.30 \mathrm{~g}$. of starting material, melting at $211^{\circ}$ with decomposition. Dilution of the mother liquor $1: 1$ with cold water precipitated a white solid, which was recovered by filtration; yield, $2.03 \mathrm{~g}$. melting at $172.5-173.5^{\circ}$. This substance wes found to be a mixture of several components, only one of which was isolated in a pure form by recrystallization of the mixture from benzene - methanol (1:4), in which this component was readily soluble. Thus, $0.24 \mathrm{~g} .(9 \%)$ of a white microcrystalline powder, melting at $212-213^{\circ}$ with decomposition, was obtained. Recrystallizations from acetone - methanol and from methanol - benzene petroleun ether raised the melting point to $215.5^{\circ}$ with decomposition. A mixed melting point with dibenzoylkojic acid $\alpha$-disemicarbazone", also melting at $215^{\circ}$ with decomposition, showed a marked depression.

Anel. Calcd. for $\mathrm{C}_{21} \mathrm{H}_{19 \mathrm{~N} 307}$ (Mol, wt. 425): C, 59.29; H, 4.47; N, 9.88. Found: $\mathrm{C}, 59.16,59.03 ; \mathrm{H}, 4.68,4.55 ; \mathrm{N}, 10.1$.

(bb) Preparation of Dibenzoylkojic Acid B-"Monosemicarbazone"

A suspension of $3.00 \mathrm{~g} \cdot(0.00622 \mathrm{~mole})$ of dibenzoylkojic acid $\alpha$-"disemicarbazone" in $60 \mathrm{ml}$. of glacial acetic acid was stirred at room temperature, while a concentrated aqueous solution of $7.20 \mathrm{~g}$. (0.104 mole) of sodium nitrite was added, drop by drop, in two portions. The addition periods of about 1 hour each were spaced 2.5 hours apart. Stirring was stopped on completion of the addition, and the reaction liquor was filtered. Only traces of the starting material had remained undissolved. The filtrate was diluted with $240 \mathrm{ml}$. of water to precipitate 
$2.41 \mathrm{~g}$. of white solid melting at $166-170^{\circ}$ with decomposition. By boilIng this materiel with $120 \mathrm{ml}$. of methanol some impuro starting material could be removed, as the latter was insoluble in methanol. The filtered extract, when diluted with $500 \mathrm{ml}$. of water, deposited $1.82 \mathrm{~g}$. (69\%) of a white microcrystalline solid melting at $178-179^{\circ}$ with decomposition. This melting point was not altered by recrystallization from benzene methanol - petroleum ether. The substance was recovered practically unchanged after its suspension in glacial acetic acid was treated with an excess of concentrated aqueous sodium nitrite at room temperature.

Anal. Calcd. for $\mathrm{C}_{21} \mathrm{H}_{1} \mathrm{NN}_{3} \mathrm{O}_{7}$ (Mol. wt. 425): C, 59.29; $\mathrm{H}, 4.47 ; \mathrm{N}$, 9.88. Found: C, 59.29, 59.21; H, 5.07, 4.91; N, 9.79.

(oc) Action of Dilute Acid on Dibenzoylkojic Acid $\alpha$-Disemicarbazone"

A suspension of $1.50 \mathrm{~g} \cdot(0.00311$ mole $)$ of dibenzoylkojic acid $\alpha$-"disemicarbazone" in $25 \mathrm{ml}$. of $10 \%$ aqueous hydrochloric acid was heated to boiling, and kept under reflux for 3.5 hours. Then the red, clear liquor was separated from the dark brown, sticky, insoluble solid material. The acid liquor yielded a total of $0.31 \mathrm{~g}$. ( $41 \%$ of 2 equivalent wts.) of a white solid melting at $121.5-122.5^{\circ} ; 0.20 \mathrm{~g}$. of this amount separated from the liquor on cooling, and $0.11 \mathrm{~g}$. was obtained by extraction with ether, followed by extraction of the ether solution with dilute aqueous sodium carbonate. A mixture of this substance with an authentic sample of benzoic acid showed no depression of the melting point. The dark brown crude solld was recrystallized from methanol ethanol - benzene to give two portions of a pale tan solid, of which 
$0.25 \mathrm{~g}$. melted at $207-210^{\circ}$ and $0.18 \mathrm{~g}$. at $203-206^{\circ}$. These two portions were combined, and purified by recrystallization from dioxane - water and from acotic acid - water, and by treatment with activated carbon in acetone followed by crystallization from acetone. None of these methods were really satisfactory, and losses were considerable. The pure product was a white microcrystalline solid with a pale yellow tinge, melting at $244-244.5^{\circ}$. Yield: $0.11 \mathrm{~g} \cdot(9 \%)$. The melting point was not raised by further recrystallizations from chloroform - petroleum ether, and from ethyl acetate - chloroform.

Anal. Calcd. for $\mathrm{C}_{21} \mathrm{H}_{15} \mathrm{~N}_{3} \mathrm{O}_{5}$ (Mol wt. 389): 0, 64.79; H, 3.86; $\mathrm{N}, 10.8$. Found: $\mathrm{C}, 64.46,64.33 ; \mathrm{H}, 4.16,4.10 ; \mathrm{N}, 10.8$; Mol. wt., 423.

(dd) Action of Dilute Alkali on Dibenzoylkojic Acid $\alpha$-Disemicarbazone"

A suspension of $2.00 \mathrm{~g} \cdot(0.00415 \mathrm{~mole})$ of dibenzoylkojic acid $\alpha$-disemicarbazone" in $20 \mathrm{ml}$. of $10 \%$ aqueous potassium hydroxide was stirred at room temperature for 30 minutes. At the end of this period only traces of solid were undissolved. After filtration the clear yellow liquor was diluted with $40 \mathrm{ml}$. of water, and extracted with ether and with chloroform, but only traces of impure material were recovered from these extracts. Then the liquor was acidified with concentrated hydrochloric acid to approximately $\mathrm{pH}$ 2. A white solid precipitate formed, which was taken up into ether in the course of the extraction of the acidic liquor with that solvent. The ether extract in turn was back-extracted with $10 \%$ qqueous sodium carbonate, and the aqueous extract acidified with concentrated hydrochloric acid, to precipitate $0.95 \mathrm{~g} \cdot(95 \%$ of 2 equiralent 
wts.) of a white solid melting at $120.5-121.5^{\circ}$. A mixture of this material with an authentic sample of benzojc acid did not show a depression of the melting point. The ether solution was evaporated after the extraction with sodium carbonate, but only traces of impure solid were recovered thereby.

(ee) Oxidation of Dibenzoylkojic Acid $\alpha$ "Disemicarbazone" with Aqueous Potassium Permanganate

A mixture of $5.61 \mathrm{~g} \cdot(0.0116 \mathrm{~mole})$ of dibenzoylkojic acid $\alpha$ - disemicarbazone", $10.00 \mathrm{~g} \cdot(0.0633 \mathrm{~mole})$ of potassium permanganate, $4 \mathrm{ml}$. $(0.07$ mole) of glacial acetic acid and $370 \mathrm{ml}$. of water was heated in an open vessel to $90^{\circ}$, and kept at that temperature for 0.5 hours. Then the brown manganese dioxide was removed by filtration, and the colorless, clear filtrate ( $\mathrm{pH}$ 6) was concentrated to $250 \mathrm{ml}$. at $60^{\circ}$ and a pressure of $20 \mathrm{~m}$. The concentrated liquor was acidified to approximately $\mathrm{pH} 2$ with concentrated sulfuric acid, and the resulting milky suspension was extracted continuously for 30 hours with benzene. The benzene extract was dried over anhydrous sodium sulfate, concentrated to a small volume and diluted with petroleum ether. An impure white solid separated and was recovered by filtration. Further concentration of the filtrate yielded $0.32 \mathrm{~g}$. of white solid melting at $112-116^{\circ}$. This fraction consiated largely of benzoic acid, while the first impure fraction proved to be mixture of benzoic acid and benzoylglycolic acid. Since the latter was only speringly soluble in hexane, in which benzoic acid was freely soluble, the two could be separated by recrystallizations from 
benzene - hexane. All benzoic acid fractions were combined and recrystallized from water to give $0.38 \mathrm{~g}$. (27\% of 1 equivalent wt.) of pure benzoic acid melting at $122-122.5^{\circ}$. The identity of this material was established by a mixed melting point determination with an authentic sample of benzoic acid. The benzoylglycolic acid fractions were purified by recrystallizations from hexane - ethyl acetate to yield $0.24 \mathrm{~g}$. (11.5\% of 1 equivalent wt.) of white needles melting at 109-111\%. Recrystallization from hexane - benzene raised the melting point ultimately to $111-112^{\circ}$, which was the melting point recorded in the literature (154). The neutralization equivalent of this sample was found to be 179.7; the calculated velue for benzoylglycolic acid is 180 .

(ff) Oxidation of Dibenzoylkojic Acid B-"Monosemicarbazone" with Aqueous Potassium Permanganate

A mixture of $0.60 \mathrm{~g} \cdot(0.00141$ mole $)$ of dibenzoylkojic acid $\beta$-"monosemicarbazone", $1.10 \mathrm{~g} \cdot(0.00696$ mole $)$ of potassium permanganate and $40 \mathrm{ml}$. of water was heated in an open vessel on a ateam bath for 1.75 hours. At the end of this period the brown precipitate of manganese dioxide was removed by filtration, the pale golden colored filtrate was acidified with concentrated hydrochloric acid to approximately $\mathrm{pH} 2$, and the acidic aqueous liquor was extracted with benzene. Evaporation of the dried benzene extract left $0.27 \mathrm{~g}$. of an impure brown-white solid, which when crystallized from hexane - benzene yielded $0.05 \mathrm{~g} \cdot(19.5 \%$ of 1 equivalent wt.) of crude benzoylglycolic acid, melting at $106-109.5^{\circ}$. Recrystallization from hexane - benzene raised the melting point to 
$111-112^{\circ}$, and also yielded some impure benzoic acid, the melting point of which could be raised by repeated recrystallizations from water to the correct velue of $121 \cdot 5-122^{\circ}$. The identity of the benzoic acid was established by a mixed melting point determination with an authentic sample, and that of the benzoylglycolic acid by a mixed melting point determination with a sample (m.p. $111-112^{\circ}$ ) obtained from the oxidation of dibenzoylkojic acid $\alpha$ - disemicarbazone" with permanganate (ee).

(gg) Dibenzoylkofic Acid $\beta$-"Monosemicarbazone" and Semicarbazide Hydrochloride

Dibenzoylkojic acid $\beta$-"monosemicarbazone", $0.20 \mathrm{~g} \cdot(0.000471$ mole), and semicarbazide hydrochloride, $0.11 \mathrm{~g} \cdot(0.000985$ mole), were suspended in a mixture of $0.12 \mathrm{~g} .(0.00152 \mathrm{~mole})$ of pyridine and $10 \mathrm{ml}$. of $80 \%$ aqueous methanol. Heating of the suspension soon caused complete dissolution of the solids, and the resulting clear solution was kept boiling under reflux for 1.33 hours. No color change occurred. The solution was then filtered, concentrated on the steam bath to $5 \mathrm{ml}$. and diluted with $30 \mathrm{ml}$. of cold water. A white crystalline precipitate which formed was recovered on a filter. Yield, $0.17 \mathrm{~g} .(85 \%)$ melting at $176-176.5^{\circ}$ with decomposition. A mixture with a sample of the starting material (m.p. $175^{\circ}$ with decomposition) did not show a depression of the melting point.

(hh) Dibenzoylkojic Acid B-"Monosemicarbazone" and Hydroxylamine Hydrochloride

A mixture of $0.10 \mathrm{~g} \cdot(0.000235$ mole $)$ of dibenzoylkojic acid 
123

$\beta$-"monosemicarbazone", $0.035 \mathrm{~g} \cdot(0.000505 \mathrm{~mole})$ of hydroxylamine hydrochloride, $0.05 \mathrm{~g} \cdot(0.000634 \mathrm{~mole})$ of pyridine, and $6 \mathrm{ml}$. of $80 \%$ aqueous methanol was heated to boiling. All the solids dissolved within a few minutes; the clear solution was kept under reflux for 3 hours, was filltared, concentrated to $4 \mathrm{ml}$. and diluted with $30 \mathrm{ml}$. of water. A crop of white crystals was recovered, weighing $0.085 \mathrm{~g} .(85 \%)$ and melting at 176-176. $5^{\circ}$ with decomposition. A mixed melting point of this substance with the starting material (mop. $175^{\circ}$ with decomposition) was not depressed. 
SUMMARY AND CLAIMS TO ORIGINAL RESEARCH

1. Contrary to expectation, kojic acid was found to be stable in an excess of normal aqueous alkali at room temperature; methylations and benzoylations conducted in this medium failed to demonstrate the presence of open chain, enolic cleavage products, and only known and new cyclic derivatives were obtained. The now derivatives were the crystaline 7-monomethyl ether (5-hydroxy-2-(methoxymethyl)- $\gamma$-pyrone), m.p. $75-76^{\circ}$, its crystalline aluminum salt, m.p. $270-271^{\circ}$ dec., and a crystalline molecular addition compound composed of one molecule each of dibenzoylkojic acid and benzoic acid, m.p. 120-121 . The stability of the addition compound was investigated under a variety of conditions.

2. Crystalline sodium kojate tetrahydrate was prepared for the first time; the usefulness of the anhydrous salt for the synthesis of certain inaccessible derivatives of kojic acid was demonstrated by preparing from it 5-benzoxy-2-(hydroxymethyl)- $\gamma$-pyrone in close to $80 \%$ yield. This derivative in turn served as starting material in the preparation of 2-(acetoxymethyl)-5-benzoxy- $\gamma$-pyrone, m.p. $144^{\circ}$, a new crystalline mixed ester of kojic acid.

3. A convenient method for the selective debenzoylation of 5-benzoates 
of kojic acid was worked out, utilizing hydroxylamine hydrochloride and pyridine at room temperature. Sensitive groups, e.g. acetate, located in the side chain of kojic acid were practically unaffected under the conditions of the debenzoylation. A new crystalline monobenzoate of kojic acid, 2-(benzoxymethyl)-5-hydroxy- $\gamma$-pyrone, m.p. $180-181^{\circ}$, was prepared in $90 \%$ yield by this procedure, and methylated by diazomethane to 2 -(benzoxymethyl)-5-methoxy- $\gamma$-pyrone, m.p. 110$111^{\circ}$, another new crystalline derivative of kojic acid. Further potential applications of the procedure were discussed.

4. The reductive fission of dibenzoylkojic acid to benzoylallomaltol and benzoic acid was achieved with zinc in glacial acetic acid, a reagent rarely used for such reductions.

5. Dibenzoylkojic acid was found to condense with two molecules of semicarbazide, to form a pair of isomeric crystalline "disemicarbazones", decomposing at $215^{\circ}$ and $172-172.5^{\circ}$ respectively. The $\alpha$-isomer was cleaved by nitrous acid to two isomeric crystalline "monosemicarbazones" decomposing at $215.5^{\circ}$ and $178-179^{\circ}$ respectively. The structures of these four new derivatives could not be established definitely, but the absence of a cyclic structure was demonstrated by analytical data, by cyclization, hydrolysis and oxidation. The cyclization of the $\alpha$-"disemicarbazone" was accompanied by the fission of one of the semicarbazone groups, and reaulted in another new crystalline derivative, m.p. $244-244.5^{\circ}$. The four "semicarbazones" were the first carbonyl derivatives, as well as the first open chain cleavage products, of kojic acid. 
6. In all, twelve new crystalline derivatives of kojic acid were prepared, analysed and characterized. Seven of these were $\gamma$-pyrones and their structures were proved unequivocally; four were open chain semicarbazones, and the last was a cyclization product of one of the semicarbazones.

7. The published melting points of $135-136^{\circ}$ for 5-benzoxy-2-(hydroxymethyl)- $\gamma$-pyrone $(4,72)$, and $206-208^{\circ}$ for 5-benzoxy-2-(triphenylmethoxymethyl)- $\gamma$-pyrone (72) were revised to $143.5-144.5^{\circ}$ and $212.5-$ $214^{\circ}$ respectively.

8. A probable explanation for the formation of 5-derivatives of kojic acid at lower concentrations of aqueous alkali, as contrasted with the formation of the 7-methyl ether at higher alkali concentrations, was found in the relative importance of the various structures contributing to the resonance hybrid of the kojate anion.

9. The apparent failure of normal aqueous alkali to open the ring of kojic acid, and the successful cleavage of the ring of dibenzoylkojic acid by semicarbazide were discussed in terms of electron displacements and reaction mechanisms. 


\section{REFERENCES}

1. H. N. Barham and B. L. Smits, Trans. Kansas Acad. Sci., 37,91 (1934).

2. K. Saito, Botan. Mag. (Tokyo), 21, 240 (1907).

3. T. Yabuta, Orig. Com. 8th Intern. Congr. Appl. Chem. (Appendix), 25, 455 (1912); Chem. Abstracts, 1, 2191 (1913).

4. T. Yabuta, J. Chem. Soc. Japan, 37, 1185, 1234 (1916); Chem. Abstracts, 17, 1475 (1923).

5. F. Traetta Mosca, Ann. chim. app1., 1, 477 (1914), Chem. Abstracts, 8. 3315 (1914).

6. Nadine Wijkman, Hoppe-Seyler's Z. physiol. Chem., 132, 104 (1924).

7. F. Traetta Mosca and M. Preti, Gazz. chim, ital., 21 II, 269 (1921).

8. T. Yabuta, J. Chem. Soc., 125, 575 (1924).

9. T. Yabuta and K. Kambe, J. Agr. Chem. Soc. Japan, 6, 516 (1930); Chem. Abstracts, 26, 1931 (1932).

10. J. W. Armit and T. J. Nolan, J. Chem. Soc., 3203 (1931).

11. A. Peratoner and G. Leonardi, Gazz. chim. ital., 30 I, 539 (1900).

12. A. Peratoner and F. C. Palazzo, Gazz. chim. ital., 36 I, 7 (1906).

13. A. Peratoner and A. Tamburello, Gazz. chim. 1tal., 36 I, 33 (1906).

14. I. S. Belonosov, Zhur. Priklad. Khim., 22, 1103 (1949); Chem. Abstracts, 45, 5650 (1951).

15. I. S. Belonosov, Zhur. Priklad. Khim., 24, 113 (1951); Chem. Zentr., 122 II, 1583 (1951).

16. K. Heyns and G. Vogelsang, Chem. Ber., 87, 13 (1954).

17. S. M. McElvain and M. A. Goese, J. Am. Chem. Soc., 65, 2233 (1943).

18. B. F. Duesel and J. V. Scudi, J. Am. Chem. Soc., 71, 1866 (1949). 
19. H. Tamiya and T. Hide, Acta Phytochim. (Japan), 4, 343 (1929); Chem. Abstracts, 24, 2496 (1930).

20. J. H. Birkinshaw, J. H. V. Charles, C. H. Lilly and H. Raistrick, Trans. Roy. Soc. (London), B 220, 127 (1931).

21. H. E. Morton, W. Kocholaty, Renate Junowicz-Kocholaty and A. Kelner, J. Bacteriol., 50, 579 (1945).

22. R. Q. Marston, Nature, 164, 961 (1949).

23. Dorothy Gill-Carey, Brit. J. Exptl. Pathol., 20, 119 (1949).

24. T. Takahashi and T. Asai, J. Agr. Chem. Soc. Japan, 2, 55, 369 (1933); Chem. Abstracts, 27, 1026, 3735 (1933).

25. K. Sakaguchi, I. Asai and Y. Ikeda, J. Agr. Chem. Soc. Japan, 19, 711 (1943); Chem. Abstracts, 42, 5508 (1948).

26. D. Barnard and F. Challenger, J. Chem. Soc., 110 (1949).

27. H. R. V. Arnstein and R. Bentley, Blochem. J. (London), 54, 517 (1953).

28. H. R. V. Arnstein and R. Bentley, Biochem. J. (London), 54, 493 (1953).

29. F. Challenger, L. Klein and T. K. Walker, J. Chem. Soc., 16 (1931).

30. H. Katagiri and K. Kitahara, Mem. Coll. Agr. Kyoto Imp. Univ., 26, 1 (1933); Chem. Abstracts, 27, 3235 (1933).

31. A. Corbellini and B. Gregorini, Gazz. chim. ital., 60, 244 (1930).

32. H. Katagiri and K. Kitahara, Bull. Agr. Chem. Soc. Japan, 2. 38 (1929); Chem. Abstracts, 24, 3813 (1930).

33. H. Tamiya, Acta Phytochim. (Japan), 6, 1 (1932); Chem. Abstracts, 26, 4627 (1932).

34. B. S. Gould, Biochem. J. (London), 22, 797 (1938).

35. F. Challenger, L. Klein and T. K. Walker, J. Chem. Soc., 1498 (1929).

36. H. N. Barhom and B. L. Smits, Ind. Eng. Chem., 28, 567 (1936).

37. O. E. May, A. J. Moyer, P. A. Wells and H. T. Herrick, J. Am. Chem. Soc., 23, 774 (1931).

38. K. Sakaguchi, J. Agr. Chem. Soc. Japan, I, 748 (1931), 8, 264 (1932); Chem. Abstracts, 27, 1379 (1933).

39. A. J. Kluyver and L. H. C. Perquin, Biochem. Z., 266, 68, 82 (1933). 
40. K. Kinoshita, Acta Phytochim. (Japan), 3, 31 (1927); Chem. Abstracts, 22, 1990 (1928).

41. M. A. Jennings and T. I. Williams, Nature, 155, 302 (1945).

42. A. Di Capua, Gazz. chim. ital., 63, 296 (1933).

43. J. L. Yuil1, Nature, 161, 397 (1948).

44. O. E. May, G. E. Ward and H. T. Herrick, Zentr. Bacteriol. Parasitenk., Abt. II, 86, 129 (1932); Chem. Abstracts, 26, 5986 (1932).

45. O. E. May, H. T. Herrick, A. J. Moyer and P. A. Wells, U. S. Pat. 2,006,086 (1935); Chem. Abstracts, 29, 5593 (1935).

46. J. W. Foster, "Ohemical Activities of Fungi," Academic Press Inc., New York, 'N. Y., 1949, p.430.

47. S. M. de Luna, Bull. soc. chim. biol., 31, 11 (1949).

48. E. F. Phares, E. H. Mosbach, F. W. Denison Jr., S. F. Carson, M. V. Long and B. A. Gwin, Ansl. Chem., 24, 660 (1952).

49. H. Tamiya, Acta Phytochim. (Japan), 3, 51 (1927); Chem. Abstracts, 22, 1990 (1928).

50. J. H. Birkinghaw and H. Raistrick, Trans. Roy. Soc. (London), B 220, 139 (1931).

51. R. Airoldi and G. Bionda, Ann. chim. appl., 22, 385 (1942).

52. H. N. Barham, Ind. Eng. Chem., Anal. Ed., 11, 31 (1939).

53. W. N. Heworth, "The Congtitution of Sugars," Edward Arnold and Co., London, 1929, p. 38 .

54. K. Naurer, Ber., 63, 25 (1930).

55. H. R. V. Arnstein and R. Bentley, J. Chem. Soc., 3436 (1951).

56. H. R. V. Arnstein and R. Bentley, Biochem. J. (London), 24, 508 (1953).

57. K. Maurer and A. Mülller, Ber., 63, 2069 (1930).

58. K. Maurer and W. Petsch, Ber., 64, 2011 (1931).

59. K. Maurer, Ber., 64, 2358 (1931).

60. K. Maurer and W. Petsch, Ber., 66, 995 (1933). 
61. K. Maurer and R. Böhme, Ber., 69, 1399 (1936).

62. K. Maurer and K. Plötner, Ber., 64, 281 (1931).

63. M. Stacey and Lilian M. Turton, J. Chem. Soc., 661 (1946).

64. H. S. Isbell, J. Research Natl. Bux. Standards, 32, 45 (1944).

65. T. Soda, T. Katura and O. Yoda, J. Chem. Soc. Japan, 61, 1227 (1940); Chem. Abstracts, 37, 4083 (1943).

66. J. Fried, in "Heterocyclic Compounds," R. C. Elderfield, ed., John Wiley and Sons, Inc., New York, N. Y., 1950, Vol. 1, p. 373.

67. J. W. Wiley, J. N. Tyson Jr, and J. S. Steller, J. Am. Chem. Soc., 64, $963(1942)$.

68. C. Musante, Gazz. chim. ital., 79, 679 (1949).

69. M. L. Moss and M. G. Mellon, Ind. Eng. Chem., Anal. Ed., 13, 612 (1941).

70. J. P. Mehlig and M. J. Shepherd Jx., Anal. Chem., 2l, 642 (1949).

71. C. D. Hurd and R. J. Sims, J. Am. Chem. Soc., 71, 2440 (1949).

72. T. Yabute and K. Anno, J. Agr. Chem. Soc. Japan, 23, 104 (1949); Ohem. Abstracts, 4ㅡ, 3492 (1950).

73. P. A. Wolf and W. M. Westveer, Arch. Biochem., 28, 201 (1950).

74. R. M. Hann, J. Am. Chem. Soc., 56, 485 (1934).

75. K. N. Campbel1, J. F. Ackerman and Barbara K. Campbell, J. Org. Chem., 15, 221 (1950).

76. J. H. Looker and T. T. Okamoto, Abstracts of papers presented before the Division of Organic Chemistry at the meeting of the American Chemical Society in Kansas City, March 23rd, 1954.

77. F. Kipnis, H. Soloway and J. Ornfelt, J. Am. Chem. Soc., $70,4264(1948)$.

78. G. H. Morey, U. S. Pat. 2,422,740 (1947); Chem. Abstracts, 41, 6900 (1947).

79. V. Ettel and J. Hebký, Collection Czechoslor. Chem. Communs., 15, $356(1950)$

80. A. Quilico and C. Musante, Gazz. chim. 1tal., 74, 26 (1944). 
81. L. L. Woods, J. Am. Chem. Soc., 74, 1107 (1952).

82. L. L. Woods, J. Am. Chem. Soc., I2, 1039 (1950).

83. L. L. Woods, J. Am. Chem. Soc., 74, 1105 (1952).

84. L. L. Woods, J. Am. Chem. Soc., 75, 3608 (1953).

85. L. L. Woods, J. Am. Chem. Soc., 75, 1510 (1953).

86. R. Barchielli, Ann, chim. appl., 30, 473 (1940).

87. L. L. Woods, J. Am. Ohem. Soc., 72, 4322 (1950).

88. L. L. Woods, J. Am. Chem. Soc., 74, 1106 (1952).

89. L. L. Woods, J. Am. Ohem. Soc., 74, 3958 (1952).

90. L. L. Woods, J. Am. Chem. Soc., 75, 2009 (1953).

91. L. L. Woods, J. Am. Chem. Soc., 74, 3959 (1952).

92. H. N. Barham and G. N. Reed, J. Am. Chem. Soc., 60, 1541 (1938).

93. H. A. Bruson, in "Organic Reactions," R. Adams, ed., John Wiley and Sons, Inc., New York, N. Y., 1949, Vol. 5, p. 79.

94. J. R. Files and F. Challenger, J. Chem. Soc., 663 (1940).

95. L. L. Woods, J. Am. Chem. Soc., 68, 2744 (1946).

96. L. F. Cavalleri, Chem. Revs., 41, 525 (1947).

97. Y. Obata and T. Yamanishi, J. Agr. Ohem. Soc. Japan, 24, 334 (1951); Chem. Abstracts, 46, 11474 (1952).

98. L. L. Woods, J. Am. Chem. Soc., 69, 2073 (1947).

99. K. Maurer, B. Schiedt and H. Schroeter, Ber., 68, 1716 (1935).

100. L. L. Woods, J. Am. Ohem. Soc., 70, 2608 (1948).

101. F. H. Pollard, J. F. W. McOmie and I. I. M. Elbeih, Nature, 163, 292 (1949).

102. A. H. Fox, Nature, 155, 397 (1945).

103. H. A. McKinstry, P. F. Eiland and R. Pepinaky, Acta cryat., 2, 285 (1952). 
104. L. P. Kuhn, Anal. Chem., 22, 276 (1950).

105. H. F. Lee, B. Boltjes and W. M. Eisenmenn, Am. Rev. Tuberc., 61, 738 (1950).

106. A. E. Oxford, J. Soc. Ohem. Ind. (London), 61, 48 (1942).

107. A. H. Cook and M. S. Lacey, Nature, 155, 790 (1945).

108. C. Arnoudi and C. Colla, Boll. soc. ital. biol. apor., 21, 164 (1946); Chem. Abstracts, 41, 3504 (1947).

109. J. W. Footer and E. O. Karow, J. Bacteriol., 49, 19 (1945).

110. F. Kavanagh, J. Bacter101., 54, 761 (1947).

111. S. D. Kromer, H. A. Greer and D. A. Szobel, J. Immunol., 49. 273 (1944).

112. Elizabeth A. Hall, F. Kavanagh and I. N. Asheshor, Antibiotice and Chemotherapy, 1, 369 (1951).

113. J. R. Klein and N. S. Olsen, J. Biol. Chem., 170, 151 (1947).

114. J. C. McGowan, P. W. Brian and H. G. Heuming, Ann. Appl. Biol., 25, 25 (1948).

115. W. C. O'Kane and G. H. Morey, U. S. Pat. 2, 460,188 (1949); Chem. Abstracts, 43, 4419 (1949).

116. E. L. Mayer, Florence B. Talley and C. F. Woodward, U. S. Dept. Agr., Bur. Entomol. Plant Quarantine, E-709 (1946); Chem. Abstracts, 41, 2528 (1947).

117. E. Gäumann and A. v. Arx, Ber. schweiz. botan. Ges., 57, 174 (1947); Chem. Abstracts, 42, 6890 (1948).

118. T. E. Friedemann, Science, 80, 34 (1934).

119. N. J. Giarman, J. Pharmacol. Expt1. Therap., 24, 232 (1948).

120. N. J. Glarman, J. Pharmacol. Exptl. Therap., 26, 119 (1949).

121. L. Haitinger and A. Lieben, Nonatsh., 5, 339 (1884).

122. L. Claisen, Ber., 24, 111 (1891).

123. R. Willstätter and R. Pummerer, Ber., 37, 3733 (1904).

124. A. Schönberg and A. Sina, J. Am. Chem. Soc., 72, 1611 (1950). 
125. R. Willstätter and R. Pummerer, Ber., 38, 1461 (1905).

126. C. D. Logan, Ph. D. Thesis, MicGill University, 1949, p. 130.

127. R. F. Hunter, A. M. Qureishy and R. Samuel, J. Chem. Soc., 1576 (1936).

128. F. Tiemann and P. Krüger, Ber., 18, 727 (1885).

129. A. Jeanrenaud, Ber., 르, 1270 (1889).

130. C. R. Hauser and W. B. Renfrow, Jr., in "Organic Syntheses," J. R. Johnson, ed., John Wiley and Sons, Inc., New York, N. Y., 1939, Vol. 19, p. 15.

131. L. W. Jones and Leonora Neuffer, J. Am. Chem. Soc., 32, 659 (1917).

132. H. L. Yale, Chem. Revs., 33, 209 (1943).

133. C. K. Ingold and C. W. Shoppee, J. Chem. Soc., 1868 (1928).

134. P. E. Verkade, C. P. van Dijk and F. A. in't Veld, Rec. trav. chim., 60, 112 (1941).

135. K. Miescher and C. Scholz, Rec. trav. chim., 60, 428 (1941).

136. W. H. Hartung and R. Simonoff, in "Organic Reactions," R. Adams, ed., John Wiley and Sons, Inc., New York, N. Y., 1953, Vol. 7, p. 263.

137. K. Kraut, Ann., 192, 222 (1878).

138. A. Quilico, I. Panizzi and E. Mugnaini, Gazz. chim. ital., 79, 89 (1949); Chem. Abstracts, 43, 7913 (1949).

139. G. Wittig, F. Vidal and E. Bohnert, Chem. Ber., 83, 366 (1950).

140. D. N. Bedekar, R. P. Kaushal and S. S. Deshapande, J. Indian Chem. Soc., 12, 465 (1935).

141. W. Borsche and Iris Bonacker, Ber., 54, 2678 (1921).

142. G. Soliman and I. E. El-Kholy, J. Chem. Soc., 1755 (1954).

143. R. Oliveri Tortorici, Gazz. chim. ital., 30 I, 524 (1900).

144. C. Ainsworth and R. G. Jones, J. Am. Chem. Soc., 76, 3172 (1954).

145. S. Goldschmidt and W. L. C. Veer, Rec. trav. chim., 65, 796 (1946). 
146. C. K. Ingold, "Structure and Mechanism in Organic Chemistry," Cornell University Press, Ithaca, N. Y., 1953, pp. 206, 214.

147. S. C. Bunce, Anal. Chem., 25, 825 (1953).

148. J. B. Niederl and V. Niederl, "Micromethods of Quantitative Organic Analysis," John Wiley and Sons, Inc., New York, N. Y., 2nd. Edition, 1948, p. 101.

149. Ibid., p. 62 .

150. Q. P. Peniston and H. Hibbert, Paper Trade J., 109, 46 (1939).

151. R. Meyer and E. Hartmann, Ber., 28, 3956 (1905).

152. F. Arndt, in "Organic Syntheses," C. R. Noller, ed., John Wiley and Sons, Inc., New York, N. Y., 1935, Vo1. 15, p. 4, Note 7.

153. H. L. Buning, Rec. trav. chim., 40, 348 (1921).

154. P. Brigl and H. Grüner, Ber., 65, 641 (1932). 